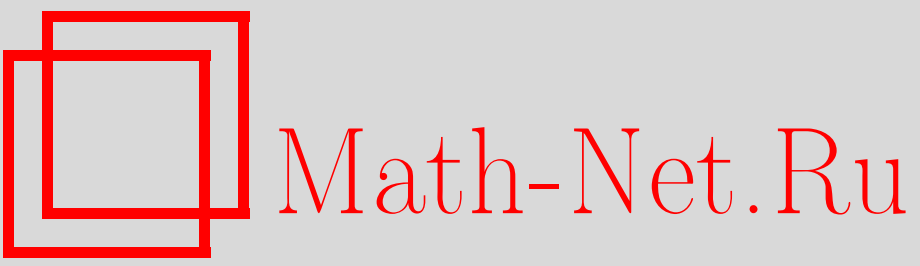

И. М. Гельфанд, М. И. Граев, В. С. Ретах, Общие гамма-функции, экспоненты и гипергеометрические функции, УМH, 1998, том 53, выпуск 1, 3-60

DOI: https://doi.org/10.4213/rm8

Использование Общероссийского математического портала Math-Net.Ru подразумевает, что вы прочитали и согласны с пользовательским соглашением

http://www.mathnet.ru/rus/agreement

Параметры загрузки:

IP: 35.173 .219 .149

26 апреля 2023 г., 14:24:49 


\title{
ОБЩИЕ ГАММА-ФУНКЦИИ, ЭКСПОНЕНТЫ И ГИПЕРГЕОМЕТРИЧЕСКИЕ ФУНКЦИИ
}

\author{
И. М. ГЕЛЬФАНД, М. И. ГРАЕВ, В. С. РЕТАХ
}

СОДЕРЖАНИЕ

Введение . . . . .

$\S 1$. Общие гамма-функции .................................... 8

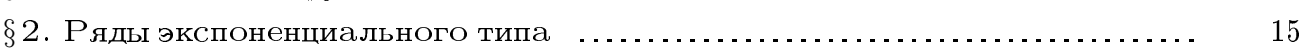

$\S 3$. Операторы $u$-дифференцирования и $u$-экспоненты ................. 23

$\S 4$. Многомерные аналоги ...................................... 34

$\S 5$. Ряды гипергеометрического типа .................................... 40

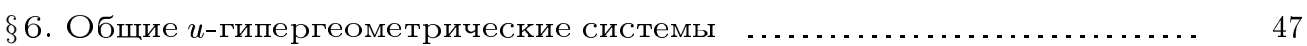

Список литературы ................................................. 58

\section{Введение}

Эта статья посвящена деформациям общих гипергеометрических функций на $\mathbb{C}^{N}$, определенных в [3] (см. также [5]). В основе лежат вводимые здесь и-гамма функиии и $и$-экспоненты одного и многих комплексных переменных.

1. Начало изучению базисных, или $q$-гипергеометрических, функций было положено Гейне (Heine E. Handbuch der Kugelfunctionen. Theorie und Anwendungen. 1878. P. 97-125). Современная теория таких функций начинается, пожалуй, с работы Аски и Вильсона [23]. В настояшее время работы на эту тему, написанные как математиками, так и физиками, насчитьваются сотнями. Книга Гаспера и Рахмана [35] содержит наиболее полный набор результатов и ссылок. Мы приводим в списке литературы лишь часть наиболее заметных статей, опубликованных после этой книги.

Современная теория $q$-специальных функций и уравнений изучает $q$-деформации их классических аналогов. Например, производная функции $d f(x) / d x$ заменяется ее $q$-деформацией, скажем,

$$
\frac{f(q x)-f\left(q^{-1} x\right)}{q x-q^{-1} x} .
$$

Работа второго автора выполнена при поддержке Российского фонда фундаментальных исследований (грант№ 95-01-01364). 
В [6], [7] рассматривались также 2-параметрические деформации производной, а именно,

$$
\left(\partial_{r, s} f\right)(x)=\frac{f(r x)-f(s x)}{x(r-s)}
$$

Исходя из теории гипергеометрических $A$-систем, рассматривались также многопараметрические деформации известных гипергеометрических уравнений. Например, наш " q-аналог уравнения Гаусса" зависит от 8 параметров. От соотношений между этими параметрами сушественно зависят свойства решений соответствуюшего уравнения, см. [6].

Мы убедились, что многие результаты этой теории сохраняются, если перейти от 1-, 2-параметрических деформаций производной к деформациям более общего вида: $(\partial f)(x)=x^{-1} u\left(x \frac{d}{d x}\right) f(x)$, где $u$ - любая целая функция. В частности, оператору $d / d x$ отвечает $u(\alpha)=\alpha$, а его 2-параметрической деформации - функция $u(\alpha)=\left(r^{\alpha}-s^{\alpha}\right) /(r-s)$. Это позволяет единым образом излагать классическую теорию и $q$-теорию и приводит к новьм аналогам классических гипергеометрических функций; естественно назвать их $u$-аналогами.

Хотя нашими основными объектами по-прежнему являются специальные функции, связанные с 2-деформациями (отдельно по каждому аргументу), мы ведем изложение на языке " $u$-анализа," которьй строим, начиная с $u$-гамма функцй и $u$-экспонент.

Классические гипергеометрические функции одного переменного и их $q$-аналоги являются $u$-экспонентами. Именно, обобщенные функции Гаусса ${ }_{r} F_{s}$ (или, по-другому, функции Похгаммера) являются $u$-экспонентами, где $u$ - рациональная функция, a их $q$-аналоги (функции Гейне) - $u$-экспонентами, где $u(\alpha)$ - рациональная функция от $q^{\alpha}$ (см. п. 21.4). Тэта-функция Якоби является (с точностью до несушественных деталей) $u$-экспонентой при $u(\alpha)=q^{-\alpha}$.

2. Чтобы построить $q$-аналоги классических функций, заменяют гамма-функцию Эйлера $\Gamma(\alpha)$ и оператор дифференцирования $d / d x$ их $q$-аналогами $\Gamma_{q}(\alpha)$ и $\partial_{q}$; по определению $\Gamma_{q}$ является решением функционального уравнения

$$
\Gamma_{q}(\alpha+1)=\frac{q^{\alpha}-1}{q-1} \Gamma_{q}(\alpha)
$$

а оператор $\partial_{q}$ задается формулой: $\left(\partial_{q} f\right)(x)=\frac{f(q x)-f(x)}{x(q-1)}$. Естественньм $q$-аналогом ряда $\exp x=\sum \frac{x^{n}}{\Gamma(n+1)}$ является ряд $\exp _{q} x=\sum \frac{x^{n}}{\Gamma_{q}(n+1)}$, которьй, как и ряд $\exp x$, обрьвается при $n<0$.

В теории квантовых групп используют другой вариант оператора $q$-дифференцирования: $\left(\partial_{q} f\right)(x)=\frac{f(q x)-f\left(q^{-1} x\right)}{x\left(q-q^{-1}\right)}$. Соответственно меняются определения функций $\Gamma_{q}(\alpha)$ и $\exp _{q}(x)$. Оба варианта - частные случаи оператора $(r, s)$-дифференцирования: $\left(\partial_{r, s} f\right)(x)=\frac{f(r x)-f(s x)}{x(r-s)}$. 
Общее свойство, роднящее операторы $\partial=\partial_{r, s}$ и $\partial=d / d x:\left[\partial, x \frac{d}{d x}\right]=\partial$. Это коммутационное соотношение означает, что на степенные функции $x^{\alpha}$ оператор $\partial$ действует по формуле $\partial x^{\alpha}=u(\alpha) x^{\alpha-1}$. Здесь $u(\alpha)=\alpha$ в случае $\partial=d / d x$, и $u(\alpha)=\frac{r^{\alpha}-s^{\alpha}}{r-s}$ в случае $\partial=\partial_{r, s}$.

3. В этой статье функция $\left(q^{\alpha}-1\right) /(q-1)$ заменяется произвольной аналитической функцией $u(\alpha)$. Соответственно, вводятся “ $u$-аналоги” гамма-функции Эйлера $\Gamma(\alpha)$, ряда $\exp x$ и оператора дифференцирования $d / d x$. Именно, $u$-гамма-функция $\Gamma_{u}(\alpha)$ есть решение функционального уравнения

$$
\Gamma_{u}(\alpha+1)=u(\alpha) \Gamma_{u}(\alpha)
$$

Формальньй ряд $\exp _{u} x$ определяется равенством

$$
\exp _{u} x=\sum_{n \in \mathbb{Z}} \frac{x^{n}}{\Gamma_{u}(n+1)}
$$

Наконец, оператор $u$-дифференцирования определяется формулой

$$
\partial_{u}=x^{-1} u\left(x \frac{d}{d x}\right)
$$

при условии, что $u$ - целая функция. Другими словами, на степенные функции $x^{\alpha}$ он действует так:

$$
\partial_{u} x^{\alpha}=u(\alpha) x^{\alpha-1} .
$$

Классические функции $\Gamma(\alpha), \exp x$ и оператор $d / d x$ отвечают случаю $u(\alpha)=\alpha, \mathrm{a}$ их $q$-аналоги - случаю $u(\alpha)=\left(q^{\alpha}-1\right) /(q-1)$.

4. Содержание статьи. В $\S \S 1-3$ исследуются введенные объекты: функции $\Gamma_{u}$, формальные ряды $\exp _{u}$ и операторы $\partial_{u}$. В $\S \S 4-6$ вводятся и изучаются $u$-гипергеометрические функции и $u$-гипергеометрические ряды многих переменных.

Функции одной переменной. В $\S 1$ для большого класса функций $u$ получены представления функций $\Gamma_{u}$ в форме бесконечных произведений и исследованы их асимптотики.

В $\S 2$ обсуждаются условия сходимости формальных рядов $\exp _{u} x$.

В $\S 3$ мы определяем $u$-экспоненты, где $u$ - произвольная целая функция, как решения уравнения

$$
\partial_{u} f=f
$$

Очевидно, формальные ряды $\exp _{v} x, v(\alpha)=u(\alpha+\lambda), \lambda \in \mathbb{C}$, удовлетворяют этому уравнению, а потому в области сходимости задают различные $u$-экспоненты. В классическом случае, когда $u(\alpha)=\alpha, \partial_{u}=d / d x$, решение $f(x)=\exp x$ единственно, с точностью до множителя. Для других $u$ существует, вообше говоря, бесчисленное множество линейно независимых $u$-экспонент. Подробно исследован случай 
$u(\alpha)=\frac{r^{\alpha}-s^{\alpha}}{r-s}$, для которого построены наборы $u$-экспонент в форме рядов и в форме интегралов. В частном случае, когда $u(\alpha)=\frac{q^{\alpha}-1}{q-1}$, все $u$-экспоненты явно описаны.

Функции многих переменных. Следуюшие три параграффа посвящены общим гипергеометрическим функциям и рядам гипергеометрического типа. Прежде чем перейти к их определению, необходимо перенести на многомерный случай определения функций $\Gamma_{u}, \exp _{u}$ и операторов $\partial_{u}$ (см. $\left.\S 4\right)$. В многомерном случае вместо одной функции $u(\alpha)$ одного переменного задается последовательность

$$
u=\left(u_{1}, \ldots, u_{N}\right)
$$

аналитических функций на $\mathbb{C}^{N}$. Предполагается, что эти функции связаны соотношениями:

$$
u_{i}(\alpha) u_{j}\left(\alpha-e_{i}\right)=u_{j}(\alpha) u_{i}\left(\alpha-e_{j}\right), \quad i, j=1, \ldots, N,
$$

где $e_{1}, \ldots, e_{N}-$ стандартный базис в $\mathbb{C}^{N}$. В частности, условие $(0.1)$ выполняется, если каждая функция $u_{i}$ зависит только от $\alpha_{i}$.

Вместо функции $\Gamma_{u}$ удобнее обобшить на многомерный случай функцию $E_{u}$, связанную с $\Gamma_{u}$ соотношением:

$$
E_{u}(\alpha)=\Gamma_{u}(\alpha+1)
$$

таким образом, функция $E_{u}(\alpha)$ является решением системы уравнений:

$$
E_{u}(\alpha)=u(\alpha) E_{u}(\alpha-1) .
$$

Многомерньй аналог функции $E_{u}$, ассоциированный с последовательностью $u=$ $\left(u_{1}, \ldots, u_{N}\right)$ функций на $\mathbb{C}^{N}$, определяется как решение системы уравнений:

$$
E_{u}(\alpha)=u_{i}(\alpha) E_{u}\left(\alpha-e_{i}\right), \quad i=1, \ldots, N .
$$

Условия (0.1) являются необходимьми условиями совместности этой системы. В частном случае, когда каждая функция $u_{i}(\alpha)$ зависит только от $\alpha_{i}$, имеем:

$$
E_{u}(\alpha)=\Gamma_{u_{1}}\left(\alpha_{1}+1\right) \cdots \Gamma_{u_{N}}\left(\alpha_{N}+1\right) .
$$

Соответственно, формальный ряд $\exp _{u} a, a \in \mathbb{C}^{N}$, определяется равенством

$$
\exp _{u} a=\sum_{k \in \mathbb{Z}^{N}} \frac{a^{k}}{E_{u}(k)}
$$

где $a^{k}=a_{1}^{k_{1}} \cdots a_{N}^{k_{N}}$. Если функции $u_{i}$ - целые, то с ними связьвается следуюший набор линейных операторов $\partial_{1}, \ldots, \partial_{N}$ в пространстве аналитических функций на $\mathbb{C}^{N}$ :

$$
\partial_{i}=a_{i}^{-1} u_{i}\left(a_{1} \frac{\partial}{\partial a_{1}}, \ldots, a_{N} \frac{\partial}{\partial a_{N}}\right), \quad i=1, \ldots, N .
$$


Другими словами, операторы $\partial_{i}$ действуют на мономы $a^{\alpha}=a_{1}^{\alpha_{1}} \cdots a_{N}^{\alpha_{N}}$ по формуле:

$$
\partial_{i} a^{\alpha}=u_{i}(\alpha) a^{\alpha-e_{i}}, \quad i=1, \ldots, N .
$$

Заметим, что условия (0.1) эквивалентны перестановочности операторов $\partial_{i}$ между собой. Экспонентами, ассоциированньми с последовательностью функций $u=$ $\left(u_{1}, \ldots, u_{N}\right)$, называются решения системы уравнений

$$
\partial_{i} f=f, \quad i=1, \ldots, N
$$

В статье для каждой последовательности $u=\left(u_{1}, \ldots, u_{N}\right)$ целых аналитических функций на $\mathbb{C}^{N}$, удовлетворяюших соотношениям (0.1), определены формальные ряды гипергеометрического типа и гипергеометрические функции.

Ряды гипергеометрического типа. Рядом гипергеометрического типа (по другому: $\Gamma$-рядом), ассоциированным с подрешеткой $\Lambda \subset \mathbb{C}^{N}$ и вектором $\lambda \in \mathbb{Z}^{N}$, называется следуюший формальный ряд:

$$
f_{\Lambda}(\lambda, a)=\sum_{l \in \Lambda} \frac{a^{l+\lambda}}{E_{u}(l+\lambda)}
$$

В частности, $f_{\mathbb{Z}^{N}}(\lambda, a)=\exp _{u} a$.

В $\S 5$ для различных последовательностей $u$ исследованы условия сходимости этих рядов. "Классический" случай, когда $u_{i}(\alpha)=\alpha_{i}, i=1, \ldots, N$, подробно рассмотрен ранее, см., например, [5].

Гипергеометрические функции. Гипергеометрической системой на $\mathbb{C}^{N}$, ассоциированной с произвольной подрешеткой $\Lambda \subset \mathbb{C}^{N}$ и вектором $\beta \in \mathbb{C}^{N} / L$, где $L \subset \mathbb{C}^{N}{ }_{-}$ натяну тое на $\Lambda$ линейное подпространство, называется следующая система уравнений в пространстве функций на $\mathbb{C}^{N}$ :

$$
\prod_{i: l_{i}>0} \partial_{i}{ }^{l_{i}} f=\prod_{i: l_{i}<0} \partial_{i}{ }^{-l_{i}} f \text { для любого } l=\left(l_{1}, \ldots, l_{N}\right) \in \Lambda,
$$

где $\partial_{i}$ - связанные с последовательностью $u=\left(u_{1}, \ldots, u_{N}\right)$ линейные операторы;

$$
\sum_{i=1}^{N} \omega^{i} a_{i} \frac{\partial f}{\partial a_{i}}=\langle\omega, \beta\rangle f
$$

для любого вектора $\omega=\left(\omega^{1}, \ldots, \omega^{N}\right) \in\left(\mathbb{C}^{N}\right)^{\prime}$, ортогонального $\Lambda$. Голоморфные решения этой системы называются $u$-гипергеометрическими функциями, ассоциированньми с $\Lambda$ и $\beta$.

В “классическом" варианте, когда $\partial_{i}=\frac{\partial}{\partial a_{i}}$, гипергеометрическая система была введена в [3] и исследована в последуюших работах. Была установлена ее голономность и построены базисы в пространствах ее решений в виде рядов гипергеометрического типа и в форме интегралов. 
Приведенные $и$-гипергеометрические функции. Любую $u$-гипергеометрическую функцию $f(a)$ на $\mathbb{C}^{N}$ можно свести к функции меньшего числа переменных. Пусть $n=\operatorname{rank} \Lambda \leqslant N, l^{1}, \ldots, l^{n}-$ произвольный фиксированный базис в подрешетке $\Lambda$, и пусть $\lambda \in \mathbb{C}^{N}-$ произвольный фиксированньй вектор из прообраза $\beta \in \mathbb{C}^{N} / L$. Тогда функцию $f$ можно представить в виде

$$
f(a)=a^{\lambda} F\left(x_{1}, \ldots, x_{n}\right),
$$

где $x_{i}=a^{l^{i}}=a_{1} l_{1}^{i} \cdots a_{N} l_{N}^{i}$ - мономы от координат $a_{1}, \ldots, a_{N}$, и $a^{\lambda}=a_{1} \lambda_{1} \cdots a_{N}{ }^{\lambda_{N}}$. Мы называем функции $F$ приведенными $u$-гипергеометрическими функциями, ассоциированными с $\Lambda$ и $\lambda$. Исходя из уравнений для $f$, можно в явном виде получить уравнения для функций $F$. Оказывается, что сушествует тесная связь между приведенными гипергеометрическими функциями и экспонентами на $\mathbb{C}^{n}$.

Отметим, что все классические гипергеометрические функции одного и многих переменных (Похгаммера, Аппеля, Лауричеллы и т. д.) являются приведенньми гипергеометрическими функциями, ассоциированными с подходяшими решетками $\Lambda$ и векторами $\lambda$.

Решения гипергеометрических систем в форме рядов и интегралов. В $\S 6$ конструкщии решений гипергеометрических систем, полученные в [3], [4] для классического случая, переносятся на общий случай. Легко убедиться, что любой формальный ряд " $u$-гипергеометрического типа"

$$
f_{\Lambda}(\lambda, a)=\sum_{l \in \Lambda} \frac{a^{l+\lambda}}{E_{u}(l+\lambda)},
$$

где $\lambda \in \mathbb{C}^{N}$ - любой вектор из прообраза $\beta \in \mathbb{C}^{N} / L$, формально удовлетворяет $u$-гипергеометрической системе. Задача состоит в том, чтобы в классе таких рядов выделить ряды с непустой областью сходимости.

Если каждая функция $u_{i}$ зависит только от $\alpha_{i}$, а $f_{u_{i}}$ - произвольная $u_{i}$-экспонента, $i=1, \ldots, N$, то $u$-гипергеометрической системе уравнений формально удовлетворяют также следуюшие интегралы:

$$
f(a)=\int_{C} \prod_{i=1}^{N} f_{u_{i}}\left(a_{i} t_{1}^{\omega_{1}^{i}} \cdots t_{r}^{\omega_{r}^{i}}\right) \prod_{j=1}^{r} t_{j}^{-\beta_{j}-1} d t_{j},
$$

где $\omega_{j}=\left(\omega_{j}^{1}, \ldots, \omega_{j}^{N}\right), j=1, \ldots, r,-$ произвольный фиксированный базис в решетке $\Lambda^{\perp} \subset\left(\mathbb{Z}^{N}\right)^{\prime}$, ортогональной $\Lambda$, и $\beta_{j}=\left\langle\omega_{j}, \beta\right\rangle$; интеграл берется по некоторому "циклу" $C \subset \mathbb{C}^{r}$ вешественной размерности $r$. Рассмотрен пример такого интеграла с непустой областью сходимости.

\section{$\S$ 1. Общие гамма-функции}

\section{1. Функциональное уравнение.}

ОПРЕДЕЛЕНИЕ. Назовем $u$-гамма-функцией, где $u(\alpha)$ - произвольная аналитическая функция, и обозначим через $\Gamma_{u}(\alpha)$ решение следуюшего функционального уравнения:

$$
\Gamma_{u}(\alpha+1)=u(\alpha) \Gamma_{u}(\alpha)
$$


Очевидно, что функция $\Gamma_{u}(\alpha)$, если она существует, определена с точностью до множителя $c(\alpha)$, являющегося периодической функцией с периодом 1. Заметим также, что любая аналитическая функция $F(\alpha)$ является $u$-гамма-функцией, где $u(\alpha)=$ $\frac{F(\alpha+1)}{F(\alpha)}$.

Классические примеры: $u(\alpha)=\alpha$ и $u(\alpha)=\frac{q^{\alpha}-1}{q-1}$. В первом случае решением уравнения (1.1) является гамма-функция Эйлера $Г(\alpha)$, а во втором - ее $q$-аналог, обычно обозначаемый через $\Gamma_{q}(\alpha)$. При $|q|<1$ функция $\Gamma_{q}$ может быть представлена в форме бесконечного произведения:

$$
\Gamma_{q}(\alpha)=(1-q)^{1-\alpha} \prod_{n=0}^{\infty} \frac{1-q^{n+1}}{1-q^{n+\alpha}}
$$

Известно [35], что $\lim _{q \rightarrow 1} \Gamma_{q}(\alpha)=\Gamma(\alpha)$.

Приведем еще два простых примера. Очевидно, если $u(\alpha) \equiv c$, то $\Gamma_{u}(\alpha)=c^{\alpha}$; если $u(\alpha)=\lambda^{\alpha}$, то $\Gamma_{u}(\alpha)=\lambda^{\frac{1}{2} \alpha(\alpha-1)}$.

1.2. Основные свойства. Из определения функции $\Gamma_{u}$ непосредственно следует, что с точностью до множителей, являюшихся периодическими функциями от $\alpha$ с периодом 1 , вьполняются следуюшие соотношения.

1) Если $v(\alpha)=u(\alpha+c)$, то $\Gamma_{v}(\alpha)=\Gamma_{u}(\alpha+c)$.

2) Если $v(\alpha)=u(-\alpha)$, то $\Gamma_{v}(\alpha)=\Gamma_{u}^{-1}(1-\alpha)$. Другими словами,

$$
\Gamma_{v}(\alpha) \Gamma_{u}(1-\alpha)=\theta(\alpha)
$$

- периодическая функция с периодом 1.

3) Если $v(\alpha)=(u(\alpha))^{\rho}$, где $\rho$ - произвольное число, то $\Gamma_{v}(\alpha)=\left(\Gamma_{u}(\alpha)\right)^{\rho}$.

4) Для любых функций $u(\alpha)$ и $v(\alpha)$ имеем:

$$
\Gamma_{u v}(\alpha)=\Gamma_{u}(\alpha) \Gamma_{v}(\alpha), \quad \Gamma_{u v^{-1}}(\alpha)=\Gamma_{u}(\alpha) \Gamma_{v}^{-1}(\alpha)
$$

В частности, если $u v=1$, то $\Gamma_{u}(\alpha) \Gamma_{v}(\alpha)-$ периодическая функция с периодом 1.

5) Если функшия $u$ представима в виде бесконечного произведения, $u=\prod_{n=1}^{\infty} u_{n}$, то $\Gamma_{u}(\alpha)=\prod_{n=1}^{\infty} \Gamma_{u_{n}}(\alpha)$ при условии, что правая часть сходится.

ПРЕДЛОЖЕНИЕ 1.1. Если $v(\alpha)=u(k \alpha), k=2,3, \ldots, m o$

$$
\Gamma_{u}(k \alpha)=\theta(\alpha) \prod_{i=0}^{k-1} \Gamma_{v}\left(\alpha+\frac{i}{k}\right)
$$

где $\theta(\alpha)-$ периодическая функция с периодом $1 / k$. 
ДоКАЗАТЕЛЬСТво. Положим

$$
\varphi(\alpha)=\Gamma_{u}(k \alpha), \quad \psi(\alpha)=\prod_{i=0}^{k-1} \Gamma_{v}\left(\alpha+\frac{i}{k}\right) .
$$

Тогда имеем:

$$
\varphi\left(\alpha+\frac{1}{k}\right)=u(k \alpha) \varphi(\alpha), \quad \psi\left(\alpha+\frac{1}{k}\right)=v(\alpha) \psi(\alpha) .
$$

Отсюда следует утверждение.

На основании приведенных свойств можно явно вычислять $u$-гамма-функции для достаточно широкого класса функций $u$.

ПримеРЫ. $1^{\circ}$. Если $u$-рациональная функция,

$$
u(\alpha)=c \frac{\prod_{i=1}^{m}\left(\alpha-a_{i}\right)}{\prod_{i=1}^{n}\left(\alpha-b_{i}\right)},
$$

TO

$$
\Gamma_{u}(\alpha)=c^{\alpha} \frac{\prod_{i=1}^{m} \Gamma\left(\alpha-a_{i}\right)}{\prod_{i=1}^{n} \Gamma\left(\alpha-b_{i}\right)},
$$

где $Г(\alpha)$ - гамма-функция Эйлера.

$2^{\circ} . u(\alpha)=\sin (s \alpha)$. Для вычисления $\Gamma_{u}(\alpha)$ воспользуемся разложением $\sin \alpha$ в бесконечное произведение:

$$
\sin (s \alpha)=s \alpha \prod_{n=1}^{\infty}\left(1-\frac{(s \alpha)^{2}}{(\pi n)^{2}}\right) .
$$

Каждому сомножителю этого произведения,

$$
u_{n}(\alpha)=1-\frac{(s \alpha)^{2}}{(\pi n)^{2}}=\frac{\left(s^{-1} \pi n+\alpha\right)\left(s^{-1} \pi n-\alpha\right)}{\left(s^{-1} \pi n\right)^{2}},
$$

отвечает функция

$$
\Gamma_{u_{n}}(\alpha)=\left(s^{-1} \pi n\right)^{1-2 \alpha} \frac{\Gamma\left(s^{-1} \pi n+\alpha\right)}{\Gamma\left(s^{-1} \pi n-\alpha+1\right)} .
$$

Из асимптотики для функции $\Gamma(\alpha)$ легко следут, что $\Gamma_{u_{n}}(\alpha)=1+O\left(1 / n^{2}\right)$ при любом фиксированном $\alpha$ общего положения, а потому бесконечное произведение $\prod_{n=1}^{\infty} \Gamma_{u_{n}}(\alpha)$ сходится. Следовательно,

$$
\Gamma_{u}(\alpha)=s^{\alpha} \Gamma(\alpha) \prod_{n=1}^{\infty}\left(\left(s^{-1} \pi n\right)^{1-2 \alpha} \frac{\Gamma\left(s^{-1} \pi n+\alpha\right)}{\Gamma\left(s^{-1} \pi n-\alpha+1\right)}\right) .
$$

$3^{\circ} . u(\alpha)=\Gamma(\alpha)$. Воспользуемся разложением $\Gamma(\alpha)$ в бесконечное произведение:

$$
\Gamma(\alpha)=\alpha^{-1} \prod_{n=1}^{\infty}\left[\left(1+\frac{1}{n}\right)^{\alpha}\left(1+\frac{\alpha}{n}\right)^{-1}\right] .
$$


Положим

$$
F_{n}(\alpha)=n^{\alpha}\left(1+\frac{1}{n}\right)^{\alpha(\alpha-1) / 2} \frac{\Gamma(n)}{\Gamma(n+\alpha)} .
$$

Очевидно, что

$$
\frac{F_{n}(\alpha+1)}{F_{n}(\alpha)}=\left(1+\frac{1}{n}\right)^{\alpha}\left(1+\frac{\alpha}{n}\right)^{-1} .
$$

Из асимптотики для функции $Г(\alpha)$ следует, что $F_{n}(\alpha)=1+O\left(1 / n^{2}\right)$ при любом фиксированном $\alpha$ общего положения, а потому бесконечное произведение $\prod_{n=1}^{\infty} F_{n}(\alpha)$ сходится. Следовательно,

$$
\Gamma_{u}(\alpha)=\Gamma^{-1}(\alpha) \prod_{n=1}^{\infty}\left[n^{\alpha}\left(1+\frac{1}{n}\right)^{\alpha(\alpha-1) / 2} \frac{\Gamma(n)}{\Gamma(n+\alpha)}\right] .
$$

Аналогично, можно воспользоваться другим разложением функции $\Gamma(\alpha)$ в бесконечное произведение,

$$
\Gamma(\alpha)=\left(\alpha e^{\gamma \alpha} \prod_{n=1}^{\infty}\left(1+\frac{\alpha}{n}\right) e^{-\alpha / n}\right)^{-1},
$$

где $\gamma$ - постоянная Эйлера-Маскерони. Из этого разложения следует:

$$
\Gamma_{u}(\alpha)=\Gamma^{-1}(\alpha) e^{-\gamma \alpha(\alpha-1) / 2} \prod_{n=1}^{\infty}\left[n^{\alpha} \frac{\Gamma(n)}{\Gamma(n+\alpha)} e^{\alpha(\alpha-1) / 2 n}\right]
$$

1.3. Разложение в бесконечное произведение. Введем для любой функции $u(\alpha)$ следуюшие обозначения:

$$
\begin{aligned}
& {[u(\alpha)]_{+\infty}=\prod_{n=0}^{\infty} u(\alpha+n),} \\
& {[u(\alpha)]_{-\infty}=\prod_{n=1}^{\infty} u(\alpha-n) .}
\end{aligned}
$$

Обозначим через $A_{+}$и $A_{-}$совокупность целых аналитических функций таких, что для любого $\alpha$ сходится соответственно бесконечное произведение (1.2) и бесконечное произведение (1.3).

В частности, если $u_{+} \in A_{+}, u_{-} \in A_{-}$, то сушествуют пределы

$$
\lim _{n \rightarrow \infty} u_{+}(\alpha+n)=1 \text { и } \lim _{n \rightarrow \infty} u_{-}(\alpha-n)=1 .
$$

Следующее утверждение непосредственно следует из определения совокупностей $A_{+}$и $A_{-}$.

ПРЕДЛОЖЕНИЕ 1.2. Если $u \in A_{+}$, mo $f_{+}(\alpha)=[u(\alpha)]_{+\infty}^{-1}$ является $u$-гаммафункиией. Аналогично, если $u \in A_{-}$, то $f_{-}(\alpha)=[u(\alpha)]_{-\infty}$ является и-гаммафункиией. 
СлЕДСТВИЕ. Если функиия и $(\alpha)$ представима в виде

$$
u(\alpha)=a \lambda^{\alpha} u_{+}(\alpha) u_{-}(\alpha),
$$

где $u_{+} \in A_{+}, u_{-} \in A_{-}$, то существует следующее решение функционального уравнения (1.1):

$$
\Gamma_{u}(\alpha)=a^{\alpha} \lambda^{\frac{1}{2} \alpha(\alpha-1)}[u(\alpha)]_{+\infty}^{-1}[u(\alpha)]_{-\infty}
$$

ПРимеР. Из асимптотической формулы для Г $(\alpha)$ следует, что функция

$$
v(\alpha)=\frac{\sqrt{2 \pi} \alpha^{\alpha-1 / 2} e^{-\alpha}\left(1+\frac{1}{12 \alpha}\right)}{\Gamma(\alpha)}
$$

принадлежит $A_{+}$и $A_{-}$. Таким образом, можно принять, например, $\Gamma_{v}(\alpha)=[u(\alpha)]_{+\infty}^{-1}$.

Найдем, исходя из этой формулы, функцию $\Gamma_{u}(\alpha)$ для $u(\alpha)=\alpha^{\alpha}$. Поскольку

$$
\alpha^{\alpha}=(2 \pi)^{-1 / 2} \alpha^{3 / 2}\left(\alpha+\frac{1}{12}\right)^{-1} e^{\alpha} \Gamma(\alpha) v(\alpha),
$$

при $u(\alpha)=\alpha^{\alpha}$ имеем:

$$
\Gamma_{u}(\alpha)=(2 \pi)^{-\alpha / 2} \Gamma^{3 / 2}(\alpha) \Gamma^{-1}\left(\alpha+\frac{1}{12}\right) e^{\frac{\alpha(\alpha-1)}{2}} \Gamma_{\Gamma}(\alpha)[v(\alpha)]_{+\infty}^{-1} ;
$$

выражение для $\Gamma_{\Gamma}(\alpha)$ см. в п. 1.2, пример $3^{\circ}$.

\section{4. Функции $\Gamma_{u}^{+}$и $\Gamma_{u}^{-}$.}

ОПРЕДЕЛЕНИЕ. Если целая функция $u(\alpha)$ представима в виде

$$
u(\alpha)=a \lambda^{\alpha} u_{+}(\alpha), \quad u_{+} \in A_{+},
$$

то обозначим через $\Gamma_{u}^{+}$следующую $u$-гамма-функцию:

$$
\Gamma_{u}^{+}(\alpha)=a^{\alpha} \lambda^{\frac{1}{2} \alpha(\alpha-1)}[u(\alpha)]_{+\infty}^{-1} .
$$

Аналогично, если

$$
u(\alpha)=b \mu^{\alpha} u_{-}(\alpha), \quad u_{-} \in A_{-},
$$

то обозначим через $\Gamma_{u}^{-}$следующую $u$-гамма-фиункцию:

$$
\Gamma_{u}^{-}(\alpha)=b^{\alpha} \mu^{\frac{1}{2} \alpha(\alpha-1)}[u(\alpha)]_{-\infty} .
$$

Отметим, что $\Gamma_{u}^{-}(\alpha)$ и $\left(\Gamma_{u}^{+}(\alpha)\right)^{-1}$ - целые функции. При этом, если $S-$ множество нулей функции $u$, то нулями функции $\Gamma_{u}^{-}(\alpha)$ являются точки $\alpha=s+n$, а нулями функции $\left(\Gamma_{u}^{+}(\alpha)\right)^{-1}$ - точки $\alpha=s-n+1$, где $s \in S$ и $n=1,2, \ldots$

Рассмотрим случай, когда функщия $u(\alpha)$ одновременно представима как в виде $(1.4)$, так и в виде (1.6). В этом случае имеется два решения функционального уравнения $(1.1)-\Gamma_{u}^{+}(\alpha)$ и $\Gamma_{u}^{-}(\alpha)$. Их отношение

$$
\theta_{u}(\alpha)=\frac{\Gamma_{u}^{-}(\alpha)}{\Gamma_{u}^{+}(\alpha)}=\left(b a^{-1}\right)^{\alpha}\left(\mu \lambda^{-1}\right)^{\frac{1}{2} \alpha(\alpha-1)}[u(\alpha)]_{+\infty}[u(\alpha)]_{-\infty}
$$

является периодической функцией с периодом 1. Назовем ее тета-функцией, ассоциированной с $u$. 
ЗАмЕчАниЕ. В классическом случае функциональному уравнению $\Gamma(\alpha+1)=$ $\alpha \Gamma(\alpha)$ удовлетворяют как гамма-функция Эйлера $\Gamma(\alpha)$, так и функция $\Gamma_{1}(\alpha)=$ $\frac{e^{\pi i \alpha}}{\Gamma(1-\alpha)}$. Можно трактовать $\Gamma_{u}^{+}(\alpha)$ и $\Gamma_{u}^{-}(\alpha)$ как аналоги функций $\Gamma(\alpha)$ и $\Gamma_{1}(\alpha)$, а равенство $\frac{\Gamma_{u}^{-}(\alpha)}{\Gamma_{u}^{+}(\alpha)}=\theta_{u}(\alpha)-$ как аналог известного соотношения

$$
\frac{\Gamma_{1}(\alpha)}{\Gamma(\alpha)} \equiv \frac{e^{\pi i \alpha}}{\Gamma(\alpha)(1-\alpha)}=\frac{1}{\pi} e^{\pi i \alpha} \sin \pi \alpha .
$$

ПРЕДЛОЖЕНИЕ 1.3. Если функиия и представима в виде (1.4) и (1.6), то при $n \rightarrow+\infty$ имеем:

$$
\begin{aligned}
& \Gamma_{u}^{+}(\alpha+n)=a^{\alpha+n} \lambda^{\frac{1}{2}(\alpha+n)(\alpha+n-1)}(1+o(1)), \\
& \Gamma_{u}^{+}(\alpha-n)=\theta_{u}^{-1}(\alpha) b^{\alpha-n} \mu^{\frac{1}{2}(\alpha+n)(\alpha+n-1)}(1+o(1)), \\
& \Gamma_{u}^{-}(\alpha-n)=b^{\alpha-n} \mu^{\frac{1}{2}(\alpha+n)(\alpha+n-1)}(1+o(1)), \\
& \Gamma_{u}^{-}(\alpha+n)=\theta_{u}(\alpha) a^{\alpha+n} \lambda^{\frac{1}{2}(\alpha+n)(\alpha+n-1)}(1+o(1)) .
\end{aligned}
$$

В самом деле, первое и третье равенство следуют непосредственно из определения функций $\Gamma_{u}^{+}$и $\Gamma_{u}^{-}$. В свою очередь, из этих равенств и из соотношения $\Gamma_{u}^{+}(\alpha \pm n)=$ $\Gamma_{u}^{-}(\alpha \pm n) \theta_{u}^{-1}(\alpha)$ следуют два других равенства.

1.5. Примеры. 1. $u_{1}(\alpha)=1-q^{\alpha+\lambda}, u_{2}(\alpha)=1-q^{-\alpha-\lambda}$, где $|q|<1$.

Так как $u_{1} \in A_{+}, u_{2} \in A_{-}$, то $\Gamma_{u_{1}}^{+}(\alpha)=\left[1-q^{\alpha+\lambda}\right]_{+\infty}, \Gamma_{u_{2}}^{-}(\alpha)=\left[1-q^{-\alpha-\lambda}\right]_{-\infty}$.

2. $u(\alpha)$ - произвольная рациональная функция от $q^{\alpha}$. Тогда $u(\alpha)$ можно представить двумя способами:

$$
u(\alpha)=a q^{k \alpha} \frac{\prod_{i=1}^{m}\left(1-q^{\alpha+\lambda_{i}}\right)}{\prod_{i=1}^{n}\left(1-q^{\alpha+\mu_{i}}\right)}=a^{\prime} q^{l \alpha} \frac{\prod_{i=1}^{m}\left(1-q^{-\alpha-\lambda_{i}}\right)}{\prod_{i=1}^{n}\left(1-q^{-\alpha-\mu_{i}}\right)},
$$

где $a^{\prime}$ и $l$ связаны с $a$ и $k$ соотношениями $a^{\prime}=(-1)^{m+n} q^{\sum \lambda_{i}-\sum \mu_{j}} a, l=k+m-n$. Из примера 1 и из свойств $u$-гамма-функций (см. п. 1.2) следует, что уравнение (1.1) имеет два решения:

$$
\begin{aligned}
& \Gamma_{u}^{+}(\alpha)=a^{\alpha} q^{\frac{k \alpha(\alpha-1)}{2}} \frac{\prod_{i=1}^{n}\left[1-q^{\alpha+\mu_{i}}\right]_{+\infty}}{\prod_{i=1}^{m}\left[1-q^{\alpha+\lambda_{i}}\right]_{+\infty}} ; \\
& \Gamma_{u}^{-}(\alpha)=a^{\prime \alpha} q^{\frac{l \alpha(\alpha-1)}{2}} \frac{\prod_{i=1}^{m}\left[1-q^{-\alpha-\lambda_{i}}\right]_{-\infty}}{\prod_{i=1}^{n}\left[1-q^{-\alpha-\mu_{i}}\right]_{-\infty}} .
\end{aligned}
$$

Используя стандартное обозначение $[a]_{\infty}=\prod_{k=0}^{\infty}\left(1-a q^{k}\right)$, эти функции можно также представить в виде:

$$
\begin{aligned}
& \Gamma_{u}^{+}(\alpha)=a^{\alpha} q^{\frac{k}{2} \alpha(\alpha-1)} \frac{\prod_{i=1}^{n}\left[q^{\alpha+\mu_{i}}\right]_{\infty}}{\prod_{i=1}^{m}\left[q^{\alpha+\lambda_{i}}\right]_{\infty}} \\
& \Gamma_{u}^{-}(\alpha)=a^{\prime \alpha} q^{\frac{1}{2} \alpha(\alpha-1)} \frac{\prod_{i=1}^{m}\left[q^{-\alpha-\lambda_{i}}\right]_{\infty}}{\prod_{i=1}^{n}\left[q^{-\alpha-\mu_{i}}\right]_{\infty}}
\end{aligned}
$$


Отношение этих функций - периодическая функция с периодом 1.

3. $u(\alpha)=\sum_{i=1}^{m} a_{i} r_{i}^{\alpha}$, где $\left|r_{1}\right|<\cdots<\left|r_{m}\right|$ (обобщение примера 1).

Представим $u(\alpha)$ в виде:

$$
u(\alpha)=a_{m} r_{m}^{\alpha} u_{+}(\alpha)=a_{1} r_{1}^{\alpha} u_{-}(\alpha),
$$

где $u_{+}(\alpha)=\left(a_{m} r_{m}^{\alpha}\right)^{-1} u(\alpha), u_{-}(\alpha)=\left(a_{1} r_{1}^{\alpha}\right)^{-1} u(\alpha)$. Очевидно, что $u_{+} \in A_{+}$, $u_{-} \in A_{-}$, а потому $u$-гамма-функциями являются следуюшие функции:

$$
\begin{aligned}
& \Gamma_{u}^{+}(\alpha)=a_{m}^{\alpha} r_{m}^{\frac{\alpha(\alpha-1)}{2}}\left[u_{+}(\alpha)\right]_{+\infty}^{-1}, \\
& \Gamma_{u}^{-}(\alpha)=a_{1}^{\alpha} r_{1}^{\frac{\alpha(\alpha-1)}{2}}\left[u_{-}(\alpha)\right]_{-\infty} .
\end{aligned}
$$

1.6. Функции $\Gamma_{r, s}(\alpha), \Gamma_{q}(\alpha)$ и $\Gamma_{q^{-1}}(\alpha)$. Рассмотрим случай, когда $u(\alpha)=$ $\frac{r^{\alpha}-s^{\alpha}}{r-s}, 0<|r|<|s|$ (частный случай примера 2 из п. 1.5). Обозначим $\Gamma_{u}^{ \pm}$и $\theta_{u}$ соответственно через $\Gamma_{r, s}^{ \pm}$и $\theta_{q}$, где $q=r / s$. Имеем:

$$
\begin{gathered}
\Gamma_{r, s}^{+}(\alpha)=(s-r)^{-\alpha} s^{\alpha(\alpha-1) / 2} \prod_{n=0}^{\infty}\left(1-q^{\alpha+n}\right)^{-1}, \\
\Gamma_{r, s}^{-}(\alpha)=(r-s)^{-\alpha} r^{\alpha(\alpha-1) / 2} \prod_{n=0}^{\infty}\left(1-q^{-\alpha+n+1}\right), \\
\theta_{q}(\alpha)=(-1)^{\alpha} q^{\alpha(\alpha-1) / 2} \prod_{n=0}^{\infty}\left(1-q^{\alpha+n}\right)\left(1-q^{-\alpha+n+1}\right) .
\end{gathered}
$$

Отметим, что, с точностью до множителя, функция $\theta_{q}(\alpha)$ совпадает с $\theta$-функцией Якоби.

Положим:

$$
\Gamma_{r, s}(\alpha) \stackrel{\text { def }}{=} \Gamma_{r, s}^{+}(\alpha)=(s-r)^{-\alpha} s^{\alpha(\alpha-1) / 2} \prod_{n=0}^{\infty}\left(1-q^{\alpha+n}\right)^{-1} .
$$

Из определения $\Gamma_{r, s}$ следует, что

$$
\begin{aligned}
& \Gamma_{r, s}(\alpha+n) \sim(s-r)^{-\alpha-n} s^{(\alpha+n)(\alpha+n-1) / 2} \text { при } n \rightarrow+\infty, \\
& \Gamma_{r, s}(\alpha+n) \sim \theta_{q}^{-1}(\alpha)(r-s)^{-\alpha-n} r^{(\alpha+n)(\alpha+n-1) / 2} \text { при } n \rightarrow-\infty .
\end{aligned}
$$

Положим, далее, в специальном случае, когда $u(\alpha)=\frac{q^{\alpha}-1}{q-1}$ и $u(\alpha)=\frac{1-q^{-\alpha}}{1-q^{-1}}$, $|q|<1$ :

$$
\begin{gathered}
\Gamma_{q}(\alpha) \stackrel{\text { def }}{=} \Gamma_{q, 1}(\alpha)=(1-q)^{-\alpha} \prod_{n=0}^{\infty}\left(1-q^{\alpha+n}\right)^{-1} \\
\Gamma_{q^{-1}}(\alpha) \stackrel{\text { def }}{=} \Gamma_{1, q^{-1}}(\alpha)=(1-q)^{-\alpha} q^{-(\alpha-1)(\alpha-2) / 2} \prod_{n=0}^{\infty}\left(1-q^{\alpha+n}\right)^{-1} .
\end{gathered}
$$


Таким образом, имеем ${ }^{1}$ :

$$
\begin{gathered}
\Gamma_{r, s}(\alpha)=s^{(\alpha-1)(\alpha-2) / 2} \Gamma_{q}(\alpha)=r^{(\alpha-1)(\alpha-2) / 2} \Gamma_{q^{-1}}(\alpha), \\
\Gamma_{q^{-1}}(\alpha)=q^{-(\alpha-1)(\alpha-2) / 2} \Gamma_{q}(\alpha) .
\end{gathered}
$$

ПРЕДЛОЖЕНИЕ 1.4. Функиии $\Gamma_{q}(\alpha)$ и $\Gamma_{q^{-1}}(\alpha)$ связаны следующим соотношением:

$$
\Gamma_{q}(\alpha) \Gamma_{q^{-1}}(1-\alpha)=(1-q)^{-1}(-q)^{1-\alpha} \theta_{q}^{-1}(-\alpha) .
$$

ДокАЗАТЕЛЬСТво. Имеем:

$$
\begin{aligned}
\Gamma_{q, 1}^{+}(\alpha) & =(1-q)^{-\alpha} \prod_{n=0}^{\infty}\left(1-q^{\alpha+n}\right)^{-1}, \\
\Gamma_{1, q^{-1}}^{-}(1-\alpha) & =\left(1-q^{-1}\right)^{\alpha-1} \prod_{n=0}^{\infty}\left(1-q^{\alpha+n}\right),
\end{aligned}
$$

откуда

$$
\Gamma_{q, 1}^{+}(\alpha) \Gamma_{1, q^{-1}}^{-}(1-\alpha)=(1-q)^{-1}(-q)^{1-\alpha} .
$$

С другой стороны,

$$
\Gamma_{1, q^{-1}}^{-}(1-\alpha)=\Gamma_{1, q^{-1}}^{+}(1-\alpha) \theta_{q}(-\alpha)
$$

Следовательно,

$$
\Gamma_{q, 1}^{+}(\alpha) \Gamma_{1, q^{-1}}^{+}(1-\alpha)=(1-q)^{-1}(-q)^{1-\alpha} \theta_{q}^{-1}(-\alpha) .
$$

СлЕДСТвИЕ. Функиии $\Gamma_{r, s} u \Gamma_{s^{-1}, r^{-1}}$ связаны соотношением:

$$
\Gamma_{r, s}(\alpha) \Gamma_{s^{-1}, r^{-1}}(1-\alpha)=(1-q)^{-1}(-r)^{1-\alpha} \theta_{q}^{-1}(-\alpha) .
$$

\section{§2. Ряды экспоненциального типа}

2.1. Ряды $\exp _{u} x$. Пусть $u(\alpha)-$ произвольная аналитическая функция и $\Gamma_{u}(\alpha)$ - отвечающая ей $u$-гамма-функция, т.е. решение функционального уравнения (1.1). Будем предполагать, что

$$
\begin{aligned}
& \Gamma_{u}(\alpha) \neq 0 \text { при } \alpha \in \mathbb{Z} ; \\
& \Gamma_{u}(\alpha) \neq \infty \quad \text { хотя бы для одного } \alpha \in \mathbb{Z} .
\end{aligned}
$$

ОПРЕДЕЛЕНИЕ. Назовем $u$-экспоненциальньм рядом и обозначим через $\exp _{u} x$ следуюший формальньй ряд:

$$
\exp _{u} x=\sum_{n=-\infty}^{+\infty} \frac{x^{n}}{\Gamma_{u}(n+1)}
$$

\footnotetext{
${ }^{1}$ Определение функции $\Gamma_{q}$ отличается от традиционного множителем $\Gamma_{q}(1)$.
} 
Условия (2.1) означают, что все коэффициенты этого ряда конечны и по крайней мере один из них отличен от нуля. Так как функция $\Gamma_{u}$ определена с точностью до множителя - периодической функции с периодом 1 , равенство $(2.2)$ определяет ряд $\exp _{u} x$ с точностью до постоянного множителя. Например, если $u(\alpha)=\alpha$, то $\exp _{u} x=c e^{x}$, где $c$ - произвольная постоянная.

Заметим, что любой формальньй ряд

$$
\sum_{n=-\infty}^{+\infty} c(n) x^{n}
$$

где $c(\alpha)$-произвольная аналитическая функция, является рядом $\exp _{u} x$, отвечающим функции $u(\alpha)=\frac{c(\alpha-1)}{c(\alpha)}$.

В дальнейшем, как правило, будут рассматриваться функции $u(\alpha)$, зависящие от дополнительного набора параметров. В этом случае связанные с ними ряды $\exp _{u} x$ также зависят от набора параметров. Например, если $u(\alpha)=\alpha+\lambda$, то $\Gamma_{u}(\alpha)=$ $\Gamma(\alpha+\lambda)$, а потому

$$
\exp _{u} x=\sum_{n=-\infty}^{+\infty} \frac{x^{n}}{\Gamma(n+\lambda+1)}
$$

В случае $\lambda \in \mathbb{Z}$ этот ряд обрывается при $n \rightarrow-\infty$; он сходится при любом $x \neq 0$ и совпадает с рядом

$$
x^{-\lambda} e^{x}=x^{-\lambda} \sum_{n=0}^{+\infty} \frac{x^{n}}{n !} .
$$

Если $\lambda \notin \mathbb{Z}$, то ряд расходится при любом $x$.

ПРЕДЛОЖЕНИЕ 2.1. Если и $(\alpha)$ не имеет особенностей при $\alpha \in \mathbb{Z}$ и выполнень условия (2.1), то либо все коэффичиенть с $(n)$ ряда (2.2) отличны от нуля, либо существует $k \in \mathbb{Z}$ такое, что $c(n) \neq 0$ при $n \geqslant k$ и $c(n)=0$ nри $n<k$.

В самом деле, если $c(m)=0$ для некоторого $m$, т.е. $\Gamma_{u}(m+1)=\infty$, то из соотношения $G_{u}(m+1)=u(m) \Gamma_{u}(m)$ и ограниченности $u(m)$ следует, что $\Gamma_{u}(m)=\infty$, и, значит, $c(m-1)=0$. Отсюда непосредственно следует утверждение.

Если все коэффициенты $c(n)$ ряда $\exp _{u} x$ отличны от нуля, то, нормировав этот ряд условием $c(0)=1$, получаем для остальных его коэффициентов следуюшие выражения:

$$
\begin{array}{ll}
c(n)=u(0) u(-1) \cdots u(n+1) & \text { при } n<0, \\
c(n)=(u(1) u(2) \cdots u(n))^{-1} & \text { при } n>0 .
\end{array}
$$

В случае, когда $c(n) \neq 0$ при $n \geqslant k$ и $c(n)=0$ при $n<k$, имеем, положив $c(k)=1$ :

$$
\exp _{u} x=x^{k}\left(1+\sum_{n=1}^{\infty} \frac{x^{n}}{u(k+1) \cdots u(k+n)}\right)
$$


Отметим, что в первом случае $u(n) \neq 0$ при любом $n \in \mathbb{Z}$, а во втором $u(k)=0$ и $u(n) \neq 0$ при $n>k$.

Область сходимости ряда (2.3) определяется асимптотикой $\Gamma_{u}(n)$ при $n \rightarrow+\infty$. Например, если $\Gamma_{u}(n) \sim c \lambda^{n}$ при $n \rightarrow+\infty$, то ряд (2.3) сходится в области $0<|x|<|\lambda|$. Если все коэффициенты ряда $\exp _{u} x$ отличны от нуля, то его область сходимости определяется асимптотикой $\Gamma_{u}(n)$ при $n \rightarrow+\infty$ и $n \rightarrow-\infty$. Например, если $\Gamma_{u}(n) \sim c_{1} \lambda_{1}^{n}$ при $n \rightarrow+\infty$ и $\Gamma_{u}(n) \sim c_{2} \lambda_{2}^{n}$ при $n \rightarrow-\infty$, то (если $\left.\left|\lambda_{2}\right|<\left|\lambda_{1}\right|\right)$ ряд $\exp _{u} x$ сходится в области $\left|\lambda_{2}\right|<|x|<\left|\lambda_{1}\right|$.

2.2. Примеры. Укажем, исходя из оценок $\S 1$, области сходимости некоторых $u$-экспоненциальных рядов.

1. $u(\alpha)=q^{-\alpha}$. В этом случае $\Gamma_{u}(\alpha)=q^{-\alpha(\alpha-1) / 2}$ и, значит,

$$
\exp _{u} x=\sum_{n \in \mathbb{Z}} q^{n(n+1) / 2} x^{n} .
$$

Очевидно, что если $|q|<1$, то ряд сходится при любом $x \neq 0$; если $|q| \geqslant 1$, то он всюду расходится.

ЗАмечАниЕ. Имеется простая связь между функцией $\exp _{u} x$ и $\theta$-функцией Якоби:

$$
\theta(w, \tau)=\sum_{n \in \mathbb{Z}} e^{\pi i n^{2} \tau+2 \pi i n w} .
$$

Именно,

$$
\exp _{u} x=\theta\left(w+\frac{\tau}{2}, \tau\right),
$$

где $q, x$ связаны с $w$ и $\tau$ соотношениями

$$
q=e^{2 \pi i \tau}, \quad x=e^{2 \pi i w} .
$$

2. $u(\alpha)$ - лорановский полином от $q^{\alpha}, 0<|q|<1$. Представим $u(\alpha)$ в виде:

$$
u(\alpha)=a q^{k \alpha} \prod_{j=1}^{m}\left(1-q^{\alpha+\lambda_{j}}\right)=a^{\prime} q^{l \alpha} \prod_{j=1}^{m}\left(1-q^{-\alpha-\lambda_{j}}\right),
$$

где $l=k+m$.

Если $u(n)=0$ хотя бы для одного $n \in \mathbb{Z}$, то ряд $\exp _{u} x$ обрывается при $n \rightarrow-\infty$; он сходится тогда и только тогда, когда $k \leqslant 0$.

Если $u(n) \neq 0$ при $n \in \mathbb{Z}$, то ряд $\exp _{u} x$ бесконечен в обе стороны; он сходится тогда и только тогда, когда $l \leqslant 0$.

3. $u(\alpha)=\sum_{i=1}^{k} a_{i} r_{i}^{\alpha}$, где $0<\left|r_{1}\right|<\left|r_{2}\right|<\cdots<\left|r_{k}\right|$. Положив $u_{+}(\alpha)=$ $a_{k}^{-1} r_{k}^{-\alpha} u(\alpha), u_{-}(\alpha)=a_{1}^{-1} r_{1}^{-\alpha} u(\alpha)$, имеем:

$$
u(\alpha)=a_{k}^{-1} r_{k}^{-\alpha} u_{+}(\alpha)=a_{1}^{-1} r_{1}^{-\alpha} u_{-}(\alpha),
$$

где $u_{+} \in A_{+}, u_{-} \in A_{-}$. 
Если $u(n)=0$ хотя бы для одного $n \in \mathbb{Z}$, то ряд $\exp _{u} x$ обрьвается при $n \rightarrow-\infty$; он сходится тогда и только тогда, когда $\left|r_{k}\right| \geqslant 1$.

Если $u(n) \neq 0$ при $n \in \mathbb{Z}$, то ряд $\exp _{u} x$ бесконечен в обе стороны; он сходится тогда и только тогда, когда $\left|r_{1}\right| \geqslant 1$.

Рассмотрим частный случай:

$$
u(\alpha)=\frac{r^{\alpha}-s^{\alpha}}{r-s}, \quad 0<|r|<|s| .
$$

В этом случае $u(0)=0$, а потому ряд $\exp _{u} x$ не содержит членов с отрищательньми степенями $n$. Он сходится в окрестности $x=0$ тогда и только тогда, когда $|s| \geqslant 1$.

2.3. Функции $\exp _{r, s} x, \exp _{q} x$ и $\exp _{q^{-1}} x$. Рассмотрим случай $u(\alpha)=\frac{r^{\alpha}-s^{\alpha}}{r-s}$, где $0<|r|<|s|$. Согласно п. 2.2 в этом случае $u$-экспоненциальньй ряд сходится и задает регулярную в окрестности $x=0$ функцию тогда и только тогда, когда $|s| \geqslant 1$. Обозначим эту функцию, нормированную условием $f(0)=1$, через $\exp _{r, s} x$. Имеем:

$$
\exp _{r, s} x=\sum_{n=0}^{\infty} \frac{x^{n}}{\Gamma_{r, s}(n+1)}
$$

где

$$
\Gamma_{r, s}(\alpha)=s^{(\alpha-1)(\alpha-2) / 2}(1-q)^{1-\alpha} \prod_{n=0}^{\infty} \frac{1-q^{1+n}}{1-q^{\alpha+n}}, \quad q=\frac{r}{s} .
$$

Обозначим далее, в соответствии с традицией, функции $\exp _{q, 1} x$ и $\exp _{1, q^{-1}} x$, где $|q|<1$, соответственно через $\exp _{q} x$ и $\exp _{q^{-1}} x$ :

$$
\begin{aligned}
\exp _{q} x & =\sum_{n=0}^{\infty} \frac{x^{n}}{\Gamma_{q}(n+1)}, \\
\exp _{q^{-1}} x & =\sum_{n=0}^{\infty} \frac{q^{n(n-1) / 2} x^{n}}{\Gamma_{q}(n+1)}
\end{aligned}
$$

где $\Gamma_{q}(\alpha)=\Gamma_{q, 1}(\alpha)$.

ПРЕДЛОЖЕНИЕ 2.2. Функции $\exp _{q} x u \exp _{q^{-1}} x$ представимы в виде бесконечных произведений

$$
\exp _{q} x=\prod_{n=0}^{\infty}\left(1-(1-q) q^{n} x\right)^{-1}
$$

$$
\exp _{q^{-1}} x=\prod_{n=0}^{\infty}\left(1+(1-q) q^{n} x\right)
$$


ДокАЗАТЕльство. Из определения функции $f(x)=\exp _{q} x$ легко следует, что она удовлетворяет уравнению

$$
\frac{f(q x)-f(x)}{(q-1) x}=f(x) .
$$

Отсюда имеем:

$$
f(x)=(1-(1-q) q x)^{-1} f(q x) .
$$

Применяя это равенство $k$ раз, получаем

$$
f(x)=\prod_{n=0}^{k}\left(1-(1-q) q^{n} x\right)^{-1} f\left(q^{k} x\right)
$$

Перейдя к пределу при $k \rightarrow \infty$, получаем равенство (2.4). Аналогично, функция $f(x)=\exp _{q^{-1}} x$ удовлетворяет уравнению

$$
\frac{f(q x)-f(x)}{(q-1) x}=f(q x)
$$

откуда $f(x)=(1+(1-q) q x) f(q x)$ и, значит,

$$
f(x)=\left(1+(1-q) q^{n} x\right) f\left(q^{k} x\right) .
$$

Перейдя к пределу при $k \rightarrow \infty$, получаем равенство (2.5).

СлЕДСТВИЕ 1. Функиия $\exp _{q^{-1}} x$ является целой, а $\exp _{q} x-$ мероморфной функиией с простыли полюсами в точках $x=(1-q)^{-1} q^{-k}, k=0,1, \ldots$.

СлЕДСТВИЕ 2. Функиии $\exp _{q} x$ u $\exp _{q^{-1}} x$ связаны соотношением

$$
\exp _{q} x \cdot \exp _{q^{-1}}(-x)=1
$$

ПРЕДЛОЖЕНИЕ 2.3. Пусть $0<q<1$. Тогда в области $|\arg x|>\varepsilon$, где $\varepsilon>0$ сколь угодно мало, имеет место оценка

$$
\left|\exp _{q} x\right|=\exp \left\{\frac{1}{2} \frac{\ln ^{2}|x|}{\ln q}+O(\ln |x|)\right\} \quad \text { npu } \quad|x| \rightarrow \infty
$$

ДокАЗАТЕЛЬСтво. Представим $x$ в виде

$$
x=(1-q)^{-1} q^{-k} \theta, \quad \theta=\theta_{1}+i \theta_{2}, \quad q \leqslant|\theta|<1 .
$$

Оценим сомножители произведения (2.4),

$$
1-(1-q) q^{n} x=1-q^{n-k} \theta
$$


Если $n<k$, то $\left|q^{n-k} \theta\right| \geqslant 1$, а потому

$$
\left|1-q^{n-k} \theta\right| \leqslant 1+q^{n-k}|\theta| \leqslant 2 q^{n-k} .
$$

$\mathrm{C}$ другой стороны, из условия $\arg \theta>\varepsilon$ следует, что $\left|\theta_{2}\right|>c|\theta|$, где $c>0$ зависит только от $\varepsilon$. Следовательно,

$$
\left|1-q^{n-k} \theta\right| \geqslant q^{n-k}\left|\theta_{2}\right| \geqslant c q^{n-k}|\theta| \geqslant c q^{n-k+1} .
$$

Итак, при $n<k$ имеем

$$
c^{-1} q^{n-k+1} \leqslant \prod_{n=0}^{k-1}\left|1-(1-q) q^{n} x\right| \leqslant 2^{k} q^{-k(k+1) / 2} .
$$

Далее, при $n=k$ имеем в силу условия $\arg \theta>\varepsilon$

$$
c_{1}^{\prime}<\left|1-(1-q) q^{n} x\right|<c_{2}^{\prime},
$$

где $c_{1}^{\prime}>0$ и $c_{2}^{\prime}>0$ зависят только от $\varepsilon$. Наконец, поскольку $\left|q^{n-k} \theta\right|<1$ при $n>k$, то очевидно, что

$$
c_{1}^{\prime \prime} \leqslant \prod_{n=k+1}^{\infty}\left|1-q^{n-k} \theta\right| \leqslant c_{2}^{\prime \prime},
$$

где $c_{1}^{\prime \prime}>0$ и $c_{2}^{\prime \prime}>0$ - некоторые константы. В результате для $\exp _{q} x$ получаем следующую оценку:

$$
C_{2} 2^{-k} q^{k(k+1) / 2} \leqslant\left|\exp _{q} x\right| \leqslant C_{1} c^{-k} q^{k(k-1) / 2},
$$

$C_{1}, C_{2}, c$ - положительные константы, а $k$ связано с $x$ соотношением (2.7). Отсюда непосредственно следует (2.6).

СледствиЕ. Функиия $\exp _{q} x$ ограничена на каждом луче $\arg x=$ const, omличном от полуоси $0<x<\infty$.

2.4. Связь с гипергеометрическими рядами от одной переменной. Рассмотрим случаи когда $u(\alpha)$ - рациональная функция от $\alpha$ и когда $u(\alpha)$ - рациональная функция от $q^{\alpha}$.

Пусть $u(\alpha)$ - рациональная функция от $\alpha$. Представим $u(\alpha)$ в виде:

$$
u(\alpha)=c \frac{\left(\alpha+\mu_{1}-1\right) \cdots\left(\alpha+\mu_{s}-1\right)}{\left(\alpha+\lambda_{1}-1\right) \cdots\left(\alpha+\lambda_{r}-1\right)},
$$

и пусть для простоты $c=1$. Тогда имеем:

$$
\Gamma_{u}(\alpha)=\frac{\Gamma\left(\alpha+\mu_{1}-1\right) \cdots \Gamma\left(\alpha+\mu_{s}-1\right)}{\Gamma\left(\alpha+\lambda_{1}-1\right) \cdots \Gamma\left(\alpha+\lambda_{r}-1\right)} .
$$


Следовательно,

$$
\exp _{u} x=\sum_{n \in \mathbb{Z}} \frac{\Gamma\left(n+\lambda_{1}\right) \cdots \Gamma\left(n+\lambda_{r}\right)}{\Gamma\left(n+\mu_{1}\right) \cdots \Gamma\left(n+\mu_{s}\right)} x^{n} .
$$

Здесь $\lambda_{i}, \mu_{j}$ играют роль параметров. Если $\lambda_{i} \notin \mathbb{Z}, i=1, \ldots, r$, то все члены этого ряда конечны. Ряд (2.8) сходится в окрестности $x=0$ тогда и только тогда, когда $r \leqslant s$ и хотя бы одно из чисел $\mu_{i}-$ целое (в этом случае ряд обрывается при $n \rightarrow-\infty$ ). Если, например, $\mu_{s}=0$, то имеем:

$$
\exp _{u} x=\sum_{n=0}^{\infty} \frac{\Gamma\left(n+\lambda_{1}\right) \cdots \Gamma\left(n+\lambda_{r}\right)}{\Gamma\left(n+\mu_{1}\right) \cdots \Gamma\left(n+\mu_{s-1}\right)} \frac{x^{n}}{n !} .
$$

Этот ряд, с точностью до множителя, зависящего только от $\lambda_{i}, \mu_{j}$, совпадает с обобщенным гипергеометрическим рядом (по другому: рядом Похгаммера), см. [10]:

$$
{ }_{r} F_{s-1}\left(\begin{array}{l}
\lambda_{1}, \ldots, \lambda_{r} \\
\mu_{1}, \ldots, \mu_{s-1}
\end{array} \mid x\right)=\sum_{n=0}^{\infty} \frac{\left(\lambda_{1}\right)_{n} \cdots\left(\lambda_{r}\right)_{n}}{\left(\mu_{1}\right)_{n} \cdots\left(\mu_{s-1}\right)_{n}} \frac{x^{n}}{n !},
$$

где $(\lambda)_{n}=\lambda(\lambda+1) \cdots(\lambda+n-1)$ при $n=1,2, \ldots,(\lambda)_{0}=1$.

Теперь рассмотрим случай, когда $u(\alpha)$ - рациональная функция от $q^{\alpha}$, где $0<|q|<1$. Представим $u(\alpha)$ в виде:

$$
u(\alpha)=c \frac{\left(1-q^{\alpha+\mu_{1}-1}\right) \cdots\left(1-q^{\alpha+\mu_{s}-1}\right)}{\left(1-q^{\alpha+\lambda_{1}-1}\right) \cdots\left(1-q^{\alpha+\lambda_{r}-1}\right)},
$$

и пусть для простоты $c=1$. Тогда имеем:

$$
\Gamma_{u}(\alpha)=\frac{\Gamma_{v}\left(\alpha+\mu_{1}-1\right) \cdots \Gamma_{v}\left(\alpha+\mu_{s}-1\right)}{\Gamma_{v}\left(\alpha+\lambda_{1}-1\right) \cdots \Gamma_{v}\left(\alpha+\lambda_{r}-1\right)},
$$

где $v(\alpha)=1-q^{\alpha}$, и, следовательно, см. $\S 1$,

$$
\Gamma_{v}(\alpha)=\prod_{k=0}^{\infty}\left(1-q^{k+\alpha}\right)^{-1} .
$$

Таким образом,

$$
\exp _{u} x=\sum_{n \in \mathbb{Z}} \frac{\Gamma_{v}\left(n+\lambda_{1}\right) \cdots \Gamma_{v}\left(n+\lambda_{r}\right)}{\Gamma_{v}\left(n+\mu_{1}\right) \cdots \Gamma_{v}\left(n+\mu_{s}\right)} x^{n}
$$

где $\Gamma_{v}(\alpha)$ задается равенством (2.9). Здесь, как и в предыдушем случае, $\lambda_{i}, \mu_{j}$ играют роль параметров.

Все члены ряда (2.10) конечны при условии, что $q^{k+\lambda_{i}} \neq 1$ при $k \in \mathbb{Z}, i=1, \ldots, r$.

Если $q^{k+\mu_{i}}=1$ хотя бы для одной пары $k \in \mathbb{Z}, i=1, \ldots, s$, то ряд (2.10) обрывается при $n \rightarrow-\infty$. Так как при этом его коэффициенты ограничены, то в отличие от 
классического ряда Похгаммера ряд (2.10) сходится в окрестности $x=0$ при любых значениях $r$ и $s$. В частности, при $\mu_{s}=1$ получаем следующий ряд, сходяшийся в окрестности $x=0$ :

$$
\exp _{u} x=\sum_{n=0}^{\infty} \frac{\Gamma_{v}\left(n+\lambda_{1}\right) \cdots \Gamma_{v}\left(n+\lambda_{r}\right)}{\Gamma_{v}\left(n+\mu_{1}\right) \cdots \Gamma_{v}\left(n+\mu_{s-1}\right)} \frac{x^{n}}{\Gamma_{v}(n+1)} .
$$

Положив в нем $e^{\lambda_{i}}=\rho_{i}, e^{\mu_{i}}=\sigma_{i}$, получаем ряд, совпадающий, с точностью до множителя, с базисным рядом Гейне [35]:

$$
{ }_{r} F_{s-1}\left[\begin{array}{l}
\rho_{1}, \ldots, \rho_{r} \\
\sigma_{1}, \ldots, \sigma_{s-1}
\end{array} \mid x\right]=\sum_{n=0}^{\infty} \frac{\left(\rho_{1}\right)_{q, n} \cdots\left(\rho_{r}\right)_{q, n}}{\left(\sigma_{1}\right)_{q, n} \cdots\left(\sigma_{s-1}\right)_{q, n}(q)_{q, n}} x^{n}
$$

где $(\rho)_{q, n}=(1-\rho)(1-\rho q) \cdots\left(1-\rho q^{n-1}\right)$.

Исследуем условия сходимости ряда (2.10) при $\mu_{i}$ обшего положения, т.е. в случае когда этот ряд бесконечен в обе стороны. Уже отмечалось, что при $n \rightarrow \infty$ коэффициенты ряда ограничены. С другой стороны, согласно п. 1.2,

$$
\Gamma_{m}(n+\alpha) \sim a(\alpha) q^{\frac{1}{2}\left[n^{2}-n(2 \alpha-1)\right]} \text { при } n \rightarrow-\infty .
$$

Таким образом, для коэффициентов ряда (2.10) имеет место оценка:

$$
c(n) \sim a q^{b n^{2}+c n} \text { при } n \rightarrow-\infty,
$$

где $b=\frac{1}{2}(r-s), c=\frac{1}{2}\left(\sum\left(2 \mu_{i}-1\right)-\sum\left(2 \lambda_{i}-1\right)\right)$. Отсюда следует, что при $r>s$ ряд сходится в окрестности $x=0$, а при $r<s$ он всюду расходится. При $r=s$ сходимость или расходимость ряда определяется числом $c$. Именно, легко убедиться, что при $\operatorname{Re} c<0$ ряд сходится в области $\left|q^{-c}\right|<|x|<1$; а в противном случае он всюду расходится.

Обобшением предыдушего примера является ряд

$$
{ }_{r} \Phi_{s}\left[\begin{array}{c}
\lambda_{1}, \ldots, \lambda_{r} \\
\mu_{1}, \ldots, \mu_{s}
\end{array} \mid x\right]=\sum_{n=-\infty}^{\infty} \frac{\Gamma_{u_{1}}\left(n+\lambda_{1}\right) \cdots \Gamma_{u_{r}}\left(n+\lambda_{r}\right)}{\Gamma_{u_{r+1}}\left(n+\mu_{1}\right) \cdots \Gamma_{u_{r+s}}\left(n+\mu_{s}\right)} x^{n}
$$

где $u_{i}(\alpha)=s_{i}^{\alpha}-r_{i}^{\alpha}, 0<\left|r_{i}\right|<\left|s_{i}\right|$.

Согласно $§ 1$ имеем:

$$
\begin{gathered}
\Gamma_{u_{i}}(\alpha)=s_{i}^{\frac{1}{2} \alpha(\alpha-1)} \prod_{k=0}^{\infty}\left(1-q_{i}^{\alpha+n}\right)^{-1}, \quad \text { где } q_{i}=\frac{r_{i}}{s_{i}} \\
\Gamma_{u_{i}}(n+\alpha) \sim c_{1} s_{i}^{\frac{1}{2}\left[n^{2}+(2 \alpha-1) n\right]} \text { при } n \rightarrow+\infty \\
\Gamma_{u_{i}}(n+\alpha) \sim c_{2} r_{i}^{\frac{1}{2}\left[n^{2}+(2 \alpha-1) n\right]} \text { при } n \rightarrow-\infty .
\end{gathered}
$$


Отсюда для коэффициентов $c(n)$ ряда (2.11) получаем следуюшие оценки:

$$
\begin{array}{ll}
c(n) \sim a_{1} b_{1}^{n^{2}} c_{1}^{n} \text { при } & n \rightarrow+\infty, \\
c(n) \sim a_{2} b_{2}^{n^{2}} c_{2}^{n} \text { при } & n \rightarrow-\infty,
\end{array}
$$

где

$$
\begin{array}{llrl}
b_{1} & =\prod_{i=1}^{r} s_{i}^{\frac{1}{2}} \prod_{i=r+1}^{r+s} s_{i}^{-\frac{1}{2}}, & b_{2} & =\prod_{i=1}^{r} r_{i}^{\frac{1}{2}} \prod_{i=r+1}^{r+s} r_{i}^{-\frac{1}{2}} \\
c_{1} & =\prod_{i=1}^{r} s_{i}^{\frac{1}{2}\left(2 \lambda_{i}-1\right)} \prod_{i=r+1}^{r+s} s_{i}^{-\frac{1}{2}\left(2 \mu_{i-r}-1\right)}, & c_{2} & =\prod_{i=1}^{r} r_{i}^{\frac{1}{2}\left(2 \lambda_{i}-1\right)} \prod_{i=r+1}^{r+s} r_{i}^{-\frac{1}{2}\left(2 \mu_{i-r}-1\right)} .
\end{array}
$$

Следствием этих оценок являются следуюшие утверждения.

$1^{\circ}$. Если ряд обрывается при $n \rightarrow-\infty$, то он сходится в окрестности $x=0$ при $b_{1} \leqslant 1$ и расходится для всех $x$ при $b_{1}>1$. При этом, если $b_{1}<1$, то он сходится для всех $x \neq 0$, а если $b_{1}=1$ - то он сходится в области $0<|x|<\left|c_{1}\right|^{-1}$.

$2^{\circ}$. Если ряд не обрьвается, то при $b_{1}<1, b_{2}>1$ он сходится при любом $x \neq 0$ и расходится, если хотя бы одно из чисел $b_{1}, b_{2}$ больше 1 . В случаях $b_{1}=1, b_{2} \leqslant 1$ и $b_{1} \leqslant 1, b_{2}=1$ сходимость ряда зависит от значений $c_{1}$ и $c_{2}$.

\section{§. Операторы $u$-дифференцирования и $u$-экспоненты}

3.1. Операторы $\partial_{u}$ и $\delta_{u}$. Переход от классического анализа к $q$-анализу основан на замене оператора дифференцирования $\partial=d / d x$ оператором $q$-дифференцирования $\partial_{q}$ :

$$
\left(\partial_{q} f\right)(x)=\frac{f(q x)-f(x)}{x(q-1)} .
$$

В теории квантовых групп обычно используют другой вариант оператора $q$-дифференцирования:

$$
\left(\partial_{q}^{\prime} f\right)(x)=\frac{f(q x)-f\left(q^{-1} x\right)}{x\left(q-q^{-1}\right)} .
$$

Обобщением этих двух операторов является оператор $(r, s)$-дифференцирования:

$$
\left(\partial_{r, s} f\right)(x)=\frac{f(r x)-f(s x)}{x(r-s)},
$$

где $r, s$ - произвольные отличные от нуля и не равные между собой числа.

Заметим, что оператор $\partial_{r, s}$ действует на степенные функции $x^{\alpha}$ по формуле:

$$
\partial_{r, s} x^{\alpha}=\frac{r^{\alpha}-s^{\alpha}}{r-s} x^{\alpha-1},
$$

и этим действием он вполне определяется.

Введем класс операторов более обшего вида, включаюший в себя как операторы $d / d x$, так и операторы $\partial_{r, s}$. 
ОПРЕДЕЛЕНИЕ. Назовем оператором $u$-дифференцирования, где $u(\alpha)-$ произвольная целая аналитическая функция, и обозначим через $\partial_{u}$ линейньй оператор в пространстве аналитических функций, действующий на степенные функции $x^{\alpha}$ по формуле:

$$
\partial_{u} x^{\alpha}=u(\alpha) x^{\alpha-1}
$$

В частности, функции $u(\alpha)=\alpha$ отвечает оператор $d / d x$, фаункции $u(\alpha)=\frac{r^{\alpha}-s^{\alpha}}{r-s}$ - оператор $\partial_{r, s}$.

Отметим, что определенные так операторы $\partial$ удовлетворяют коммутационному соотношению

$$
\left[\partial, x \frac{d}{d x}\right]=\partial .
$$

ОПРЕДЕЛЕНИЕ. Обозначим через $\delta_{u}$, где $u(\alpha)$ - произвольная целая аналитическая функция, линейный оператор в пространстве аналитических функций, действуюший на степенные функции $x^{\alpha}$ по формуле:

$$
\delta_{u} x^{\alpha}=u(\alpha) x^{\alpha}
$$

В частности, функции $u(\alpha)=\alpha$ отвечает оператор $x \frac{d}{d x}$.

В силу определения операторы $\partial_{u}$ и $\delta_{u}$ связаны соотношением:

$$
\delta_{u}=x \partial_{u}
$$

Очевидно, что операторы $\delta_{u}$, отвечаюшие различным функциям $u$, перестановочны между собой.

ЗАМЕЧАНИЕ. Если

$$
u(\alpha)=\sum_{n=0}^{\infty} \frac{u^{(n)}(0)}{n !} \alpha^{n}
$$

- разложение в ряд целой функции $u(\alpha)$, то

$$
\delta_{u}=\sum_{n=0}^{\infty} \frac{u^{(n)}(0)}{n !}\left(x \frac{d}{d x}\right)^{n}
$$

По этой причине естественно обозначать оператор $\delta_{u}$ также через $u\left(x \frac{d}{d x}\right)$ и называть $u(\alpha)$ символом этого оператора. 
Примеры. 1. Если $u(\alpha)=P(\alpha)$, где $P$-произвольный полином, то $\delta_{u}=P\left(x \frac{d}{d x}\right)$.

2. Если $u(\alpha)=\sum a_{i} q_{i}^{\alpha}$, то оператор $\delta_{u}$ задается формулой: $\left(\delta_{u} f\right)(x)=\sum a_{i} f\left(q_{i} x\right)$.

3.2. Свойства операторов $\partial_{u}$ и $\delta_{u}$. Перечислим несколько свойств операторов $\partial_{u}$ и $\delta_{u}$, которые следуют непосредственно из их определения.

1. Если $u(\alpha)=c_{1} u_{1}(\alpha)+c_{2} u_{2}(\alpha)$, где $c_{1}, c_{2} \in \mathbb{C}$, то

$$
\partial_{u}=c_{1} \partial_{u_{1}}+c_{2} \partial_{u_{2}}, \quad \delta_{u}=c_{1} \delta_{u_{1}}+c_{2} \delta_{u_{2}} .
$$

2. Для любых целых функций $u_{1}, u_{2}$

$$
\delta_{u_{1} u_{2}}=\delta_{u_{1}} \delta_{u_{2}}, \quad \partial_{u_{1} u_{2}}=\partial_{u_{1}} \circ x \circ \partial_{u_{2}} .
$$

3. Если $v(\alpha)=u(\alpha+c)$, то

$$
\delta_{v}=x^{-c} \circ \delta_{u} \circ x^{c}, \quad \partial_{v}=x^{-c} \circ \partial_{u} \circ x^{c} .
$$

4. Если $\varphi(x)=f(c x)$, то

$$
(\delta \varphi)(x)=(\delta f)(c x), \quad(\partial \varphi)(x)=c(\partial f)(c x) .
$$

\section{3. $u$-экспоненты.}

ОПРЕДЕЛЕНИЕ. Назовем и-әкспонентами или $и$-әкспонентами, ассоциированныцми с оператором $\partial_{u}$, аналитические функции $f(x)$, инвариантные относительно $\partial_{u}$ :

$$
\partial_{u} f=f
$$

Если $\partial_{u}=d / d x$, то, с точностью до множителя, функция $\exp x$ является единственным решением уравнения (3.1). Для произвольных операторов $\partial_{u}$ имеется, вообще говоря, бесконечное множество линейно независимых решений уравнения (3.1). В этом параграфе будут построены различные классы таких решений.

Сначала найдем формальные решения уравнения (3.1), заданные формальными рядами вида

$$
f_{\alpha}(x)=\sum_{n \in \mathbb{Z}} c(n) x^{n+\alpha}, \quad \alpha \in \mathbb{C} .
$$

Подставив этот ряд в уравнение, получаем следуюшее соотношение для его коэффициентов:

$$
u(n+\alpha) c(n)=c(n-1), \quad n \in \mathbb{Z} .
$$


Отсюда следует, что при фиксированном $\alpha$ ненулевое решение вида (3.2) уравнения (3.1) сушествует тогда и только тогда, когда в последовательности $\alpha, \alpha+1$, $\ldots, \alpha+n, \ldots$ содержится не более чем конечное число нулей функции $u$.

Если $u(\alpha+k) \neq 0$ при любом $k \in \mathbb{Z}$, то все коэффициенты ряда (3.2) отличны от нуля, и в предположении, что $c(0)=1$, они задаются равенствами

$$
\begin{array}{lll}
c(n)=u(\alpha) u(\alpha-1) \cdots u(\alpha-n+1) & \text { при } & n<0, \\
c(n)=(u(\alpha+1) \cdots u(\alpha+n))^{-1} & \text { при } & n>0 .
\end{array}
$$

Если же последовательность $\alpha, \alpha+1, \ldots, \alpha+n, \ldots$ содержит хотя бы один нуль функции $u$, то пусть $k \in \mathbb{Z}$-наибольшее число, для которого $u(\alpha+k)=0$. Тогда, приняв $c(k)=1$, получаем:

$$
f_{\alpha}(x)=x^{\alpha+k}+\sum_{n=1}^{\infty} \frac{x^{\alpha+k+n}}{u(\alpha+k+1) \cdots u(\alpha+k+n)} .
$$

ОПРЕДЕЛЕНИЕ. Предположим, что для заданной функции $u$ существует $u$-гаммафункция $\Gamma_{u}(\alpha)$, и что функция $\Gamma_{u}(\alpha)$ не имеет нулей. Обозначим через $\exp _{u}(\alpha, x)$ следуюший формальный ряд, зависящий от $\alpha$ как от параметра:

$$
\exp _{u}(\alpha, x)=\sum_{n \in \mathbb{Z}} \frac{x^{n+\alpha}}{\Gamma_{u}(n+\alpha+1)} .
$$

$\mathrm{B}$ частности, $\exp _{u}(0, x)=\exp _{u} x$, где $\exp _{u} x-u$-экспоненщиальный ряд, определенный в $§ 2$.

Очевидно, этот ряд отличен от нуля тогда и только тогда, когда $\Gamma_{u}(n+\alpha+1) \neq \infty$ хотя бы для одного $n \in \mathbb{Z}$ или, что равносильно, $u(n+\alpha+1) \neq 0$ хотя бы для одного $n \in \mathbb{Z}$.

Из определения оператора $\partial_{u}$ и функции $\Gamma_{u}$ следует, что ряд (3.6) удовлетворяет уравнению (3.1). Значит, при сделанном предположении об $u$-гамма-функции любой формальный ряд вида (3.2), удовлетворяюший уравнению (3.1), совпадает с точностью до множителя с рядом $\exp _{u}(\alpha, x)$.

Сходимость или расходимость ряда $\exp _{u}(\alpha, x)$ зависит от асимптотики $u(\alpha \pm n)$ при $n \rightarrow \infty$ и от расположения нулей функции $u(\alpha)$.

Для любого фиксированного $\alpha \in \mathbb{C}$ возможны два варианта.

1) Множество $\{\alpha+n \mid n \in \mathbb{Z}\}$ содержит нули функции $u(\alpha)$. В этом случае ряд $\exp _{u}(\alpha, x)$ обрывается при $n \rightarrow-\infty$, и его сходимость или расходимость зависит только от поведения $u(\alpha+n)$ при $n \rightarrow+\infty$. Если, например,

$$
u(\alpha+n) \sim a \lambda^{\alpha+n} \text { при } n \rightarrow+\infty,
$$

то очевидно, что ряд $\exp _{u}(\alpha, x)$ сходится в окрестности $x=0$ тогда и только тогда, когда $|\lambda| \geqslant 1$. При этом его радиус сходимости равен $\infty$, если $|\lambda|>1$, и $|a|$ в случае, когда $|\lambda|=1$. 
2) Множество $\{\alpha+n \mid n \in \mathbb{Z}\}$ не содержит нулей функции $u(\alpha)$. В этом случае все коэффициенты $c(n)$ ряда отличны от нуля, и его сходимость или расходимость зависит от поведения $u(\alpha+n)$ при $n \rightarrow \pm \infty$. Если, например,

$$
u(\alpha+n) \sim a \lambda^{\alpha+n} \text { при } n \rightarrow+\infty, \quad u(\alpha+n) \sim b \mu^{\alpha+n} \text { при } n \rightarrow-\infty,
$$

то ряд имеет непустую область сходимости только в следующих двух случаях:

$$
\text { a) }|\lambda| \geqslant 1,|\mu| \geqslant 1 \text { и }|\lambda \mu|>1 ; \quad \text { b) }|\lambda|=|\mu| \geqslant 1,|b|<|a| \text {. }
$$

При этом, если $|\lambda|>1,|\mu|>1$, он сходится при всех $x \neq 0$; если $|\lambda|=1,|\mu|>1$ при $0<|x|<|a|$; если $|\lambda|>1,|\mu|=1$ - при $|x|>|b|$; и если $|\lambda|=|\mu|=1$ - при $|b|<|x|<|a|$.

\section{ПРИМЕР.}

$$
u(\alpha)=\frac{r^{\alpha}-s^{\alpha}}{r-s}, \quad 0<|r|<|s|
$$

В этом случае нулями функции $u(\alpha)$ являются точки $\alpha_{k}=\frac{2 \pi i k}{\ln (r / s)}, k \in \mathbb{Z}$. Следовательно, если $\alpha=\alpha_{k}$, то ряд $\exp _{u}(\alpha, x)$ сходится тогда и только тогда, когда $|s| \geqslant 1$. Если $\alpha \neq \alpha_{k}, k \in \mathbb{Z}$, то ряд $\exp _{u}(\alpha, x)$ сходится тогда и только тогда, когда $|r| \geqslant 1$. Таким образом, если $|s|<1$, то ряд $\exp _{u}(\alpha, x)$ расходится при любом значении параметра $\alpha$.

3.4. Описание всех $u$-экспонент при $u(\alpha)=\frac{q^{ \pm \alpha}-1}{q^{ \pm 1}-1}, 0<|q|<1$.

ПРЕДЛОЖЕНИЕ 3.1. В случае, когда $u(\alpha)=\frac{q^{\alpha}-1}{q-1}$ или $u(\alpha)=\frac{1-q^{-\alpha}}{1-q^{-1}}$, әде $0<|q|<1$, любая и-экспонента имеет, соответственно, вид

$$
f(x)=c(x) \exp _{q} x \quad u \quad f(x)=c(x) \exp _{q^{-1}} x,
$$

где $\exp _{q} x u \exp _{q^{-1}} x-$ функиии, определеннье в $n .2 .3$, а $c(x)-$ произвольная функиия, удовлетворяющая условию периодичности:

$$
c(q x)=c(x)
$$

ДокАЗАтельство. Рассмотрим для определенности случай $u(\alpha)=\frac{q^{\alpha}-1}{q-1}$. Согласно п. 3.3 функция $\exp _{q} x$ является $u$-экспонентой. Из условия $c(q x)=c(x)$ следует, что $f(x)=c(x) \exp _{q} x$ также является $u$-экспонентой. Обратно, пусть $f(x)$ - произвольная $u$-экспонента. Положим $c(x)=f(x) / \exp _{q} x$, т.е. $f(x)=c(x) \exp _{q} x$. Тогда, с одной стороны,

$$
\begin{aligned}
\left(\partial_{u} f\right)(x) & =x^{-1}(q-1)^{-1}\left[c(q x) \exp _{q}(q x)-c(x) \exp _{q} x\right] \\
& =c(q x) \exp _{q}(x)+\frac{c(q x)-c(x)}{(q-1) x} \exp _{q}(x) .
\end{aligned}
$$


С другой стороны, $\left(\partial_{u} f\right)(x)=c(x) \exp _{q} x$. Отсюда получаем:

$$
(c(q x)-c(x))\left(1+x^{-1}(q-1)^{-1}\right) \exp _{q} x=0 ;
$$
следовательно, $c(q x)=c(x)$. Для случая $u(\alpha)=\frac{1-q^{-\alpha}}{1-q^{-1}}$ доказательство аналогич-
но.

3.5. $u$-экспоненты при $u(\alpha)=\frac{r^{\alpha}-s^{\alpha}}{r-s}$, где $0<|r|<|s|$. Рассмотрим формальные ряды $\exp _{u}(\alpha, x)$, формально удовлетворяюшие уравнению $\partial_{u} f=f$, где $u(\alpha)=\frac{r^{\alpha}-s^{\alpha}}{r-s}$. Согласно п. 3.3, если $|s| \geqslant 1$, то при $\alpha=\frac{2 \pi i k}{\ln (r / s)}, k \in \mathbb{Z}$, ряд $\exp _{u}(\alpha, x)$ сходится в окрестности $x=0$ и задает тем самым $u$-экспоненту. Если, кроме того, $|r| \geqslant 1$, то ряд $\exp _{u}(\alpha, x)$ сходится при любом $\alpha$; таким образом, в этом случае имеется континуальное семейство линейно независимых $u$-экспонент. Если $|s| \leqslant 1$, то $u$-экспонент, заданных сходяшимися рядами $\exp _{u}(\alpha, x)$, не сушествует.

ЗАмЕчАнИЕ. Для любого $u$ ряды $\exp _{u}(\alpha, x)$ удовлетворяют условию $\exp _{u}(\alpha+k, x)$ $=\exp _{u}(\alpha, x)$ для любых $\alpha \in \mathbb{C}$ и $k \in \mathbb{Z}$. Легко убедиться, что в случае, когда $u(\alpha)=\frac{r^{\alpha}-s^{\alpha}}{r-s}$, для любого $\alpha_{k}=\frac{2 \pi i k}{\ln (r / s)}, k=0,1, \ldots$, выполняется соотношение:

$$
\exp _{u}\left(\alpha+\alpha_{k}, x\right)=c_{k} x^{\alpha_{k}} \exp \left(\alpha, s^{-\alpha_{k}} x\right),
$$

где $c_{k}=(1-q)^{\alpha_{k}} s^{-\alpha_{k}\left(\alpha_{k}-1\right) / 2}$.

ПРЕДЛОЖЕнИЕ 3.2. Если $u(\alpha)=\frac{r^{\alpha}-s^{\alpha}}{r-s}$, әде $0<r<s<1$, то существует и-экспонента, заданная интегралом

$$
F_{c}(x)=\int_{-\infty+i c}^{+\infty+i c} \exp \left(\frac{t^{2}}{2 \ln s}-\frac{t}{2}\right) \exp _{q}\left(x e^{t}\right) d t
$$

где $q=r / s, \exp _{q}-q$-әкспонента, определенная в $n .2 .3, c-$ произвольное вещественное число.

ДокАЗАТЕЛЬСтво. Из оценки (2.6) для функции $\exp _{q} x$ легко следует, что интеграл (3.7) сходится длявсех точек $x$, не принадлежаших лучу $l_{c}=\{x \in \mathbb{C} \mid \arg x=-c\}$, и задает аналитическую функцию на комплексной плоскости с разрезом вдоль этого луча.

Убедимся, что $F_{c}$ является экспонентой. Для этого заметим, что если $f(x)=$ $\exp _{q}(a x)$, то

$$
\left(\partial_{u} f\right)(x)=\frac{\exp _{q}(q s a x)-\exp _{q}(\operatorname{sax})}{(q-1) s x}=a f(s x) .
$$

В силу этого соотношения имеем:

$$
\left(\partial_{u} F_{c}\right)(x)=\int_{-\infty+i c}^{+\infty+i c} \exp \left(\frac{t^{2}}{2 \ln s}+\frac{t}{2}\right) \exp _{q}\left(s x e^{t}\right) d t .
$$

Сделав замену переменной $t \mapsto t-\ln s$, получаем, что $\partial_{u} F_{c}=F_{c}$. 
ЗАмЕчАния. 1) Легко убедиться, что для любых близких $c_{1}, c_{2}, c_{1} \leqslant c_{2}$, и любого $x \in \mathbb{C}$ такого, что $\arg x$ не принадлежит отрезку $\left[c_{1}, c_{2}\right]$, мы имеем $F_{c_{1}}(x)=F_{c_{2}}(x)$. $\mathrm{C}$ другой стороны, функции $F_{c}$ и $F_{c+2 \pi}$ имеют одну и ту же область определения, но $F_{c} \neq F_{c+2 \pi}$. Таким образом, равенство (3.7), где $c$ принимает любые вещественные значения, задает многозначную аналитическую функцию $F$ на $\mathbb{C}$ с точкой ветвления $x=0$.

2) Несложньм вычислением получаем, что при фиксированном $q=r / s$

$$
\lim _{s \rightarrow 1-0}(2 \pi|\ln s|)^{-1 / 2} F_{c}(x)=\exp _{q}(x) .
$$

ПрЕДЛОЖЕнИЕ 3.3. Если и - функиия, определенная в предложении 3.2, то для нее существует и-экспонента, заданная следующим сходящимся рядом:

$$
\varphi(x)=\sum_{n=0}^{\infty} c_{n} \exp \left(\frac{\ln ^{2}\left((1-q) q^{n} x\right)}{2 \ln s}+\frac{1}{2} \ln ^{2}\left((1-q) q^{n} x\right)\right),
$$

где $c_{0}=0$ и $c_{n}=(-1)^{n} q^{n(n+1) / 2} \prod_{k=1}^{n}\left(1-q^{k}\right)^{-1}$ nрu $n>0$.

Доказательство следует из предложения 3.2. Именно, обозначим через $P(t)$ подынтегральную функцию в (3.7). Поскольку полюсами $\exp _{q} t$ являются точки $(1-q)^{-1} q^{-n}$, то $P(t)$ имеет полюсы в точках $t_{n}$, определенных равенствами

$$
x e^{t_{n}}=(1-q)^{-1} q^{-n}, \quad n=0,1,2, \ldots,
$$

т.е. $t_{n}=-\ln \left((1-q) q^{n} x\right)$. Отсюда следует, что при достаточно большом $c>0$

$$
\frac{1}{2 \pi i}\left(F_{-c}-F_{c}\right)=\sum_{n=0}^{\infty} \operatorname{Res}_{t=t_{n}} P(t) .
$$

Так как левая часть этого равенства является $u$-экспонентой, то $u$-экспонентой является и стоящий справа ряд. Найдем явное выражение для его коэффициентов. Из представления (2.4) функции $\exp _{q} x$ в виде бесконечного произведения следует, что вычет $\exp _{q} x$ в точке $(1-q)^{-1} q^{-n}$ равен $\prod_{k=1}^{n}\left(1-q^{-k}\right) \prod_{k=1}^{\infty}\left(1-q^{k}\right)^{-1}$, т.е. равен

$$
c(-1)^{n} q^{n(n+1) / 2} \prod_{k=1}^{n}\left(1-q^{k}\right)^{-1}, \quad \text { где } c=\prod_{k=1}^{\infty}\left(1-q^{k}\right)^{-1} .
$$

Таким образом, с точностью до множителя, полученный ряд имеет вид (3.8).

ПРЕДЛОЖЕНИЕ 3.4. Если $f(x)$ - произвольная и-әкспонента, где

$$
u(\alpha)=\frac{r^{\alpha}-s^{\alpha}}{r-s},
$$

то функиии

$$
f_{k}(x)=x^{\frac{2 \pi i k}{\ln q}} f\left(s^{-\frac{2 \pi i k}{\ln q}} x\right), \quad k \in \mathbb{Z},
$$

әде $q=r / s$, также являются $и$-экспонентами. 
ДокАЗАТЕльСТВо. Положив $\frac{2 \pi i k}{\ln q}=\alpha$, имеем:

$$
\left(\partial_{u} f_{k}\right)(x)=x^{\alpha} \frac{r^{\alpha} f\left(r s^{-\alpha} x\right)-s^{\alpha} f\left(s s^{-\alpha} x\right)}{(r-s) x} .
$$

Поскольку $(r / s)^{\alpha}=1$, отсюда следует:

$$
\left(\partial_{u} f_{k}\right)(x)=x^{\alpha} \frac{f\left(r s^{-\alpha} x\right)-f\left(s s^{-\alpha} x\right)}{(r-s) s^{-\alpha} x}=x^{\alpha} f\left(s^{-\alpha} x\right)=f_{k}(x) .
$$

Итак, если $u(\alpha)=\frac{r^{\alpha}-s^{\alpha}}{r-s}$, то с каждой $u$-экспонентой $f(x)$ связано линейное пространство $u$-экспонент, линейно порожденное функциями $f_{k}(x), k \in \mathbb{Z}$. Элементы этого пространства назовем экспонентами, родственными и-экспоненте $f$.

СлЕДСТвиЕ. В случае, когда $s=q^{k}, k \in \mathbb{Z}$, или, что то же самое, $r^{k}=s^{k+1}$, пространство и-экспонент, родственных и-экспоненте $f(x)$, состоит из функций вида $c(x) f(x)$, где $c(x)$ - произвольная функиия, удовлетворяющая условию периодичности.

Отсюда и из предложения 3.1 следует, что если $(r, s)=(q, 1)$ или $\left(1, q^{-1}\right)$, то все $u$-экспоненты родственны соответственно экспоненте $\exp _{q} x$ или $\exp _{q^{-1}} x$.

Добавление. Приведем другое доказательство предложения 3.3 , не опирающееся не предложение 3.2. Введем функции

$$
\varphi_{n}(x)=\exp \left(\frac{\ln ^{2}\left((1-q) q^{n} x\right)}{2 \ln s}+\frac{1}{2} \ln ^{2}\left((1-q) q^{n} x\right)\right),
$$

зависяшие от $q=r / s$ и $s$ как от параметров. Заметим, что $\varphi_{n}(x)=\varphi_{0}\left(q^{n} x\right)$.

Из определения $\varphi_{n}$ непосредственно следует:

ЛЕммА. Функиии $\varphi_{n}$ удовлетворяют соотношениям:

$$
\begin{aligned}
& \varphi_{n}(s x)=s(1-q) q^{n} x \varphi_{n}(x), \\
& \varphi_{n}(r x)=s(1-q) q^{n+1} x \varphi_{n+1}(x) .
\end{aligned}
$$

СлЕДСТВиЕ.

$$
\partial_{u} \varphi_{n}=q^{n} \varphi_{n}-q^{n+1} \varphi_{n+1} .
$$

В самом деле,

$$
\begin{aligned}
\partial_{u} \varphi_{n} & =s^{-1}(1-q)^{-1} x^{-1}\left(\varphi_{n}(s x)-\varphi_{n}(r x)\right) \\
& =s^{-1}(1-q)^{-1} x^{-1}\left(s(1-q) q^{n} x \varphi_{n}(x)-s(1-q) q^{n+1} x \varphi_{n+1}(x)\right) \\
& =q^{n} \varphi_{n}-q^{n+1} \varphi_{n+1} .
\end{aligned}
$$


Будем искать $u$-экспоненту в форме ряда

$$
\varphi(x)=\sum_{n=0}^{\infty} c_{n} \varphi_{n}(x)
$$

Подставив этот формальньй ряд в уравнение $\partial_{u} \varphi=\varphi$, получаем на основании (3.11) следуюшее соотношение для коэффициентов $c_{n}$ :

$$
c_{n}\left(1-q^{n}\right)=-c_{n-1} q^{n}
$$

Решая его, получаем, положив $c_{0}=1$,

$$
c_{n}=(-1)^{n} q^{n(n+1) / 2} \prod_{k=1}^{n}\left(1-q^{k}\right)^{-1} \text { при } n>0 .
$$

ЗАмечАниЕ. Ряд (3.8) сходится и задает $u$-экспоненту также и в случае, когда $s>r>1$.

В самом деле, в этом случае из явных выражений для $c_{n}$ и $\varphi_{n}$ вытекают оценки:

$$
\left|c_{n}\right|=O\left(\exp \left\{\frac{\ln q}{2} n^{2}\right\}\right), \quad\left|u_{n}(x)\right|=O\left(\exp \left\{\frac{\ln ^{2} q}{2 \ln s} n^{2}\right\}\right)
$$

Значит,

$$
\left|c_{n} u_{n}(x)\right|=O\left(e^{a n^{2}}\right),
$$

где

$$
a=\frac{1}{2}\left(\ln q+\frac{\ln ^{2} q}{\ln s}\right)=\frac{1}{2} \frac{\ln q \ln r}{\ln s} .
$$

Так как $a<0$ при $s>r>1$, отсюда следует утверждение.

Отметим, что если $r<1<s$, то из тех же оценок следует расходимость ряда (3.8).

3.6. $u$-экспоненты при $u(\alpha)=\alpha s^{\alpha-1}, s>0$. Функция $u(\alpha)$ возникает как предел функции $\frac{r^{\alpha}-s^{\alpha}}{r-s}$ при $r \rightarrow s$. Отвечаюший ей оператор $\partial_{u}$ имеет следуюший вид:

$$
\partial_{u} f(x)=f^{\prime}(s x)
$$

Если $s=1$, то единственньм, с точностью до множителя, решением уравнения (3.12) является функция ехр $x$. Здесь будет рассмотрен случай $s \neq 1$. Будет показано, что в этом случае имеется бесконечно много решений уравнения (3.12), и будут описаны некоторые из этих решений.

Заметим, что $u$-гамма-функция имеет следующий вид:

$$
\Gamma_{u}(\alpha)=s^{(\alpha-1)(\alpha-2) / 2} \Gamma(\alpha)
$$


где $\Gamma(\alpha)$ - гамма-функция Эйлера. Таким образом, формальное решение $f_{s}(\alpha, x)=$ $\exp _{u}(\alpha, x)$ уравнения $(3.12)$ задается формулой:

$$
f_{s}(\alpha, x)=\sum_{n \in \mathbb{Z}} s^{-(n+\alpha)(n+\alpha-1) / 2} \frac{x^{n+\alpha}}{\Gamma(n+\alpha+1)} .
$$

Очевидно, что при $s>1$ для любого $\alpha \in \mathbb{C}$ ряд (3.13) сходится при всех $x \neq 0$ и задает тем самым $u$-экспоненту. В частности, имеется единственная, с точностью до множителя, $u$-экспонента, регулярная в точке $x=0$ :

$$
f_{s}(x)=\sum_{n=0}^{\infty} s^{-n(n-1) / 2} \frac{x^{n}}{n !} .
$$

При $s<1 u$-экспонент вида (3.13) не сушествует.

ПРЕДЛОЖЕНИЕ 3.5. Функиия $f_{s}(x)$, заданная сходящимся рядом $(3.14)$, имеет следующее интегральное представление:

$$
f_{s}(x)=(2 \pi \ln s)^{-\frac{1}{2}} \int_{-\infty}^{+\infty} \exp \left(-\frac{\left(t+\frac{i}{2} \ln s\right)^{2}}{2 \ln s}+e^{i t} x\right) d t .
$$

ДокАЗАТЕЛЬСтво. Очевидно, что функция $f_{s}$, заданная равенством $(3.15)$, целая, и легко убедиться, что при $x=0$ значения функщий (3.15) и (3.14) совпадают (равны 1). Поэтому достаточно убедиться, что $f_{s}^{\prime}(s x)=f_{s}(x)$. Имеем:

$$
\begin{aligned}
f_{s}^{\prime}(s x) & =(2 \pi \ln s)^{-\frac{1}{2}} \int_{-\infty}^{+\infty} \exp \left(-\frac{\left(t+\frac{i}{2} \ln s\right)^{2}}{2 \ln s}+i t+i t s x\right) d t \\
& =(2 \pi \ln s)^{-\frac{1}{2}} \int_{-\infty}^{+\infty} \exp \left(-\frac{\left(t-\frac{i}{2} \ln s\right)^{2}}{2 \ln s}+e^{i(t-i \ln s)} x\right) d t \\
& =(2 \pi \ln s)^{-\frac{1}{2}} \int_{-\infty-i \ln q}^{+\infty-i \ln q} \exp \left(-\frac{\left(t+\frac{i}{2} \ln s\right)^{2}}{2 \ln s}+e^{i t} x\right) d t
\end{aligned}
$$

Очевидно, что интеграл (3.15) сохраняется при замене пути интегрирования $(-\infty,+\infty)$ на $(-\infty-i c,+\infty-i c)$, где $c-$ любое вешественное число. Следовательно, $f^{\prime}(s x)=f(x)$.

ПримечАниЕ. Установим связь между $u$-экспонентами $f_{s}(\alpha, x)$, заданными рядом $(3.13)$, и $\theta$-фрункцией $\theta(z, \tau)$ :

$$
\theta(z, \tau)=\sum_{n \in \mathbb{Z}} \exp \left(\pi i n^{2} \tau+2 \pi i n z\right)
$$

Определим функцию $\varphi_{s}(\alpha, x)$ равенством

$$
\varphi_{s}(\alpha, x)=\int_{0}^{\infty} e^{-t} f_{s}(\alpha, t x) d t
$$


Так как $\int_{0}^{\infty} e^{-t} t^{n+\alpha} d t=\Gamma(n+\alpha+1)$, то

$$
\begin{aligned}
\varphi_{s}(\alpha, x) & =\sum_{n \in \mathbb{Z}} s^{-(n+\alpha)(n+\alpha-1) / 2} x^{n+\alpha} \\
& =s^{-\alpha(\alpha-1) / 2} x^{\alpha} \sum_{n \in \mathbb{Z}} s^{-n^{2} / 2}\left(s^{-\alpha+1 / 2} x\right)^{n} .
\end{aligned}
$$

Сравнивая это выражение с выражением для $\theta(z, \tau)$, заключаем:

$$
s^{\alpha(\alpha-1) / 2} x^{-\alpha} \varphi_{s}(\alpha, x)=\theta(z, \tau),
$$

где $z, \tau$ связаны с $x, s$ соотношениями:

$$
q=e^{-2 \pi i \tau}, \quad q^{-\alpha+1 / 2} x=e^{2 \pi i z}
$$

ПРЕДЛОЖЕНИЕ 3.6. При $s<1$ существует и-экспонента, заданная следующим интегралом:

$$
F_{c}(x)=\int_{-\infty}^{+\infty} \exp \left(\frac{t^{2}}{2 \ln s}-\frac{t}{2}+x e^{t}\right) d t
$$

где с - произвольное вещественное число.

ДокАЗАТЕльство. То, что (3.16) удовлетворяет уравнению $\partial_{u} f=f$, легко проверить непосредственно. Отметим, что (3.16) можно получить также из (3.7) предельным переходом при $r \rightarrow s$ (поскольку $\lim _{q \rightarrow 1} \exp _{q} x=\exp x$ ).

Подобно функции, заданной интегралом (3.7), равенство (3.16) при произвольном $c$ задает многозначную функцию и тем самым определяет бесконечно много $u$-экспонент.

ПРЕДЛОЖЕНИЕ 3.7. $\lim _{s \rightarrow 1+0} f_{s}(x)=\exp x, \quad \lim _{s \rightarrow 1-0}(2 \pi \ln s)^{-1 / 2} F_{c}(s, x)=$ $\exp x$.

ДокаЗАТЕльство. Первое равенство очевидно. Докажем второе равенство. Заменой переменных $t \mapsto t|\ln s|^{1 / 2}$ получаем

$$
(2 \pi \ln s)^{-1 / 2} F_{c}(x)=(2 \pi)^{-1 / 2} \int_{-\infty+i c^{\prime}}^{+\infty+i c^{\prime}} \exp \left(-\frac{t^{2}}{2}-\frac{t}{2}|\ln s|+x e^{t|\ln s|}\right) d t
$$

Отсюда следует, что

$$
\lim _{s \rightarrow 1-0}(2 \pi \ln s)^{-1 / 2} F_{c}(s, x)=(2 \pi)^{-1 / 2} \int_{-\infty+i c^{\prime}}^{+\infty+i c^{\prime}} \exp \left(-\frac{t^{2}}{2}+x\right) d t=\exp x .
$$




\section{§4. Многомерные аналоги}

В $\S \S 1-3$ мы связали с каждой аналитической функцией $u(\alpha)$ одного переменного $u$-гамма-функцию $\Gamma_{u}$, формальньй ряд $u$-экспоненциального типа и (при условии, что $u$ - целая функция) линейньй оператор $\partial_{u}$ в пространстве аналитических функций. Перенесем эти понятия на многомерньй случай.

В многомерном варианте вместо одной функции $u(\alpha), \alpha \in \mathbb{C}$, фиксируется последовательность

$$
u=\left(u_{1}, \ldots, u_{N}\right)
$$

аналитических функций на $\mathbb{C}^{N}$.

4.1. Функция $E_{u}$. Обобщим на многомерньй случай функцию $E_{u}$, связанную с $u$-гамма-функцией одного переменного соотношением

$$
E_{u}(\alpha)=\Gamma_{u}(\alpha+1)
$$

Таким образом, $E_{u}-$ это решение функционального уравнения

$$
E_{u}(\alpha)=u(\alpha) E_{u}(\alpha-1)
$$

ОПРЕДЕЛЕНИЕ. Обозначим через $E_{u}(\alpha)$, где $u=\left(u_{1}, \ldots, u_{N}\right)$ - набор аналитических функций на $\mathbb{C}^{N}$, ненулевую функцию на $\mathbb{C}^{N}$, удовлетворяющую следующей системе уравнений:

$$
E_{u}(\alpha)=u_{j}(\alpha) E_{u}\left(\alpha-e_{j}\right), \quad j=1, \ldots, N
$$

где $\left\{e_{j}\right\}-$ стандартньй базис в $\mathbb{C}^{N}$.

Очевидно, что функция $E_{u}(\alpha)$, если она сушествует, определена однозначно с точностью до множителя - периодической функщии с периодом 1 относительно каждого переменного $\alpha_{j}$.

ЗАмечАниЕ. Любая аналитическая функция $F(\alpha)$ является функцией $E_{u}(\alpha), u=$ $\left(u_{1}, \ldots, u_{N}\right)$, где $u_{j}(\alpha)=\frac{F(\alpha)}{F\left(\alpha-e_{j}\right)}$. См. в [57] описание всех функций $F(\alpha)$, для которых $\frac{F(\alpha)}{F\left(\alpha-e_{j}\right)}, j=1, \ldots, N,-$ рациональные функции.

ПРЕДЛОЖЕНИЕ 4.1. Для существования ненулевого решения уравнений (4.1) необходимо, чтобь функиии и

$$
u_{i}(\alpha) u_{j}\left(\alpha-e_{i}\right)=u_{j}(\alpha) u_{i}\left(\alpha-e_{j}\right), \quad i, j=1, \ldots, N
$$

Доказательство очевидно.

В дальнейшем предполагается, что условие (4.2) всегда вьполнено. 
Очевидно, что условия (4.2) заведомо вьполняются в случае, когда для каждого $j=1, \ldots, N$ функщия $u_{j}$ зависит только от $\alpha_{j}$, и в этом случае решением уравнений (4.1) является функщия

$$
E_{u}(\alpha)=\prod_{j=1}^{N} E_{u_{j}}\left(\alpha_{j}\right)=\prod_{j=1}^{N} \Gamma_{u_{j}}\left(\alpha_{j}+1\right)
$$

где $\Gamma_{u_{j}}$ - гамма-функция одного переменного, введенная в $§ 1$.

Подобно функциям одного переменного, функции $E_{u}$ обладают следующими свойствами.

1) Если $v(\alpha)=u(\alpha+c), c \in \mathbb{C}^{N}$, то

$$
E_{v}(\alpha)=E_{u}(\alpha+c)
$$

2) Если последовательность $u=\left(u_{1}, \ldots, u_{N}\right)$ удовлетворет условиям (4.2), то последовательность $v=\left(v_{1}, \ldots, v_{N}\right)$, где $v_{j}(\alpha)=u_{j}\left(-\alpha+e_{j}\right), j=1, \ldots, N$, также удовлетворет условиям (4.2), и

$$
E_{v}(\alpha)=E_{u}^{-1}(-\alpha)
$$

3) Для любых $u=\left(u_{1}, \ldots, u_{N}\right)$ и $v=\left(v_{1}, \ldots, v_{N}\right)$ имеем:

$$
E_{u \cdot v}(\alpha)=E_{u}(\alpha) E_{v}(\alpha), \quad E_{u \cdot v^{-1}}(\alpha)=E_{u}(\alpha) E_{v}^{-1}(\alpha)
$$

где $u \cdot v=\left(u_{1} v_{1}, \ldots, u_{N} v_{N}\right)$.

4.2. Функция $[u(\alpha)]_{k}$. Обозначим для любой последовательности аналитических функций на $\mathbb{C}^{N} u=\left(u_{1}, \ldots, u_{N}\right)$, удовлетворяюших условию $(4.2)$, и любого $k \in \mathbb{Z}^{N}$ :

$$
[u(\alpha)]_{k}=\frac{E_{u}(\alpha)}{E_{u}(\alpha-k)}
$$

В частности, $[u(\alpha)]_{0}=1,[u(\alpha)]_{e_{j}}=u_{j}(\alpha), j=1, \ldots, N$.

ПРЕДЛОЖЕНИЕ 4.2. Для любых $k^{\prime}, k^{\prime \prime} \in \mathbb{Z}^{N}$ имеем:

$$
[u(\alpha)]_{k^{\prime}+k^{\prime \prime}}=[u(\alpha)]_{k^{\prime}}\left[u\left(\alpha-k^{\prime}\right)\right]_{k^{\prime \prime}}
$$

В самом деле, (4.4) следует из тождества:

$$
\frac{E_{u}(\alpha)}{E_{u}\left(\alpha-k^{\prime}-k^{\prime \prime}\right)}=\frac{E_{u}(\alpha)}{E_{u}\left(\alpha-k^{\prime}\right)} \frac{E_{u}\left(\alpha-k^{\prime}\right)}{E_{u}\left(\alpha-k^{\prime}-k^{\prime \prime}\right)} .
$$


СлеДСТвиЕ. $[u(\alpha)]_{-k}=\left([u(\alpha+k)]_{k}\right)^{-1}$.

Найдем для любого $k \neq 0$ явное выражение функции $[u(\alpha)]_{k}$ через функции $u_{j}(\alpha)$, $j=1, \ldots, N$. В простейшем случае $N=1$ имеем

$$
\begin{aligned}
& {[u(\alpha)]_{k}=u(\alpha) u(\alpha-1) \cdots u(\alpha-k+1) \quad \text { при } k>0,} \\
& {[u(\alpha)]_{k}=(u(\alpha) u(\alpha+1) \cdots u(\alpha+|k|))^{-1} \text { при } k<0 .}
\end{aligned}
$$

Пусть теперь $N>1$. Назовем путем из 0 в $k=\left(k_{1}, \ldots, k_{N}\right)$ произвольную последовательность элементов из $\mathbb{Z}^{N}$,

$$
k^{0}, k^{1}, \ldots, k^{r},
$$

такую, что $k^{0}=0, k^{r}=k$ и $k^{i}=k^{i-1}+\varepsilon_{i} e_{\sigma(i)}, i=1, \ldots, r$, где $\varepsilon_{i}= \pm 1$, а $\sigma-$ некоторое отображение из $[1, r]$ в $[1, N]$. Заметим, что кратчайший путь из 0 в $k$ имеет длину $r=|k|=\sum\left|k_{i}\right|$, и для любого такого пути $k_{\sigma(i)} \neq 0$ и $\varepsilon_{i}=\operatorname{sign} k_{\sigma(i)}$.

ПРеДЛОЖЕНИЕ 4.3. Для любого пути $k^{0}, \ldots, k^{r}$ из 0 в $k$ имеем:

$$
[u(\alpha)]_{k}=\prod_{i=1}^{r} v_{\sigma(i)}(\alpha)
$$

əде $v_{\sigma(i)}(\alpha)=u_{\sigma(i)}\left(\alpha-k^{i-1}\right)$ nри $\varepsilon_{i}=1 ; v_{\sigma(i)}(\alpha)=u_{\sigma(i)}^{-1}\left(\alpha-k^{i}\right)$ nри $\varepsilon_{i}=-1$.

В самом деле, (4.5) следует из тождества:

$$
\frac{E_{u}(\alpha)}{E_{u}(\alpha-k)}=\prod_{i=1}^{r} \frac{E_{u}\left(\alpha-k^{i-1}\right)}{E_{u}\left(\alpha-k^{i}\right)}=\prod_{i=1}^{r} \frac{E_{u}\left(\alpha-k^{i-1}\right)}{E_{u}\left(\alpha-k^{i-1}-\varepsilon_{i} e_{\sigma(i)}\right)} .
$$

4.3. Ряды $\exp _{u} x$. Пусть $u=\left(u_{1}, \ldots, u_{N}\right)$ - произвольньй набор аналитических функций на $\mathbb{C}^{N}$, удовлетворяющих соотношениям (4.2), и $E_{u}$ - ассоциированная с этим набором функция, т.е. решение системы уравнений (4.1). Предположим, что

$$
E_{u}(\alpha) \neq 0 \text { при } \alpha \in \mathbb{Z}^{N} ; \quad E_{u}(\alpha) \neq \infty \text { хотя бы для одного } \alpha \in \mathbb{Z}^{N} .
$$

ОПРЕДЕЛЕНИЕ. Назовем $u$-экспоненциальным рядом и обозначим через $\exp _{u} x$ следуюший формальньй ряд:

$$
\exp _{u} x=\sum_{k \in \mathbb{Z}^{N}} \frac{x^{k}}{E_{u}(k)}, \quad x \in \mathbb{C}^{N},
$$

где $x^{k}=x_{1}^{k_{1}} \cdots x_{N}^{k_{N}}$.

Условия (4.6) означают, что все коэффищиенты этого ряда конечны, и по крайней мере один из них отличен от нуля. Так как функция $E_{u}$ определена с точностью до множителя - периодической функции с периодом 1 относительно каждого аргумента $\alpha_{j}$, то равенство (4.7) определяет ряд $\exp _{u} x$ с точностью до постоянного множителя. 
В специальном случае, когда каждая функция $u_{j}, j=1, \ldots, N$, зависит только от $\alpha_{j}$, имеем: $\exp _{u} x=\prod_{j=1}^{N} \Gamma_{u_{j}}\left(\alpha_{j}+1\right)$. Поэтому

$$
\exp _{u} x=\prod_{j=1}^{N} \sum_{k_{j} \in \mathbb{Z}}\left(\frac{x_{j}^{k_{j}}}{\Gamma_{u_{j}}\left(k_{j}+1\right)}\right)=\prod_{j=1}^{N} \exp _{u_{j}} x_{j}
$$

где $\exp _{u_{j}} x_{j}-u_{j}$-экспоненщиальный ряд одной переменной.

Если все коэффищиенты ряда $\exp _{u} x$ отличны от нуля, то, нормировав $E_{u}$ условием $E_{u}(0)=1$, мы можем представить ряд $\exp _{u} x$ в следуюшем виде:

$$
\sum_{k \in \mathbb{Z}^{N}} \frac{x^{k}}{[u(k)]_{k}}=\sum_{k \in \mathbb{Z}^{N}}[u(0)]_{-k} x^{k},
$$

где $[u(k)]_{k}$ задается равенством (4.5).

ЗАмЕчАнИЕ. Любой формальньй ряд

$$
\sum_{k \in \mathbb{Z}^{N}} c(k) x^{k}, \quad x \in \mathbb{C}^{N}
$$

где $c(\alpha)$ - произвольная аналитическая функция на $\mathbb{C}^{N}$, является рядом $\exp _{u} x$, где $u=\left(u_{1}, \ldots, u_{N}\right), u_{j}(\alpha)=\frac{c(\alpha)}{c\left(\alpha-e_{j}\right)}$.

4.4. Операторы $\delta_{v}$ и $\partial_{v, j}$. Свяжем с каждой целой аналитической функцией $v(\alpha)$ на $\mathbb{C}^{N}$ линейный оператор $\delta_{v}$ в пространстве аналитических функций на $\mathbb{C}^{N}$, действуюший на мономы $a^{\alpha}=a_{1}^{\alpha_{1}} \cdots a_{N}^{\alpha_{N}}, \alpha \in \mathbb{C}^{N}$, по формуле

$$
\delta_{v} a^{\alpha}=v(\alpha) a^{\alpha} .
$$

Очевидно, что операторы $\delta_{v}$, отвечающие различным функциям $v$, перестановочны между собой и обладают следуюшими свойствами:

$1^{\circ} . \delta_{v^{\prime} v^{\prime \prime}}=\delta_{v^{\prime}} \delta_{v^{\prime \prime}}$ для любых целых функций $v^{\prime}$ и $v^{\prime \prime}$;

$2^{\circ}$. если $v^{\prime}(\alpha)=v(\alpha+c), c \in \mathbb{C}^{N}$, то

$$
\delta_{v^{\prime}}=a^{-c} \circ \delta_{v} \circ a^{c}
$$

$3^{\circ}$. если $\varphi(a)=f(c a), c \in \mathbb{C}^{N}$, где $c a=\left(c_{1} a_{1}, \ldots, c_{N} a_{N}\right)$, то

$$
\left(\delta_{v} \varphi\right)(a)=\left(\delta_{v} f\right)(c a) .
$$


ЗАмЕчАнИЕ. Подобно одномерному случаю естественно обозначать $\delta_{v}$ через

$$
v\left(a \frac{\partial}{\partial a}\right)
$$

где $a \frac{\partial}{\partial a}=\left(a_{1} \frac{\partial}{\partial a_{1}}, \ldots, a_{N} \frac{\partial}{\partial a_{N}}\right)$, и назьвать $v(\alpha)$ символом оператора $\delta_{v}$.

Определим, далее, линейные операторы $\partial_{v}, j$ в пространстве аналитических функший на $\mathbb{C}^{N}$ формулой

$$
\partial_{v, j}=a_{j}^{-1} \circ \delta_{v}, \quad j=1, \ldots, N .
$$

Таким образом, операторы $\partial_{v, j}$ действуют на мономы $a^{\alpha}=a_{1}^{\alpha_{1}} \cdots a_{N}^{\alpha_{N}}$ по формуле:

$$
\partial_{v, j} a^{\alpha}=v(\alpha) a^{\alpha-e_{j}} .
$$

Отметим несколько элементарных свойств операторов $\partial_{v, j}$ :

$1^{\circ} \cdot\left[\partial_{v, j}, a_{i} \frac{\partial}{\partial a_{i}}\right]=\delta_{i, j} \partial_{v, j}$

$2^{\circ}$. если $v(\alpha)=u(\alpha+c), c \in \mathbb{C}^{N}$, то $\partial_{v, j}=a^{-c} \circ \partial_{u, j} \circ a^{c}$;

$3^{\circ}$. если $\varphi(a)=f(c a), c \in \mathbb{C}^{N}$, то $\left(\partial_{v, j} \varphi\right)(a)=c_{j}\left(\partial_{v, j} f\right)(c a)$.

Пусть $u=\left(u_{1}, \ldots, u_{N}\right)$ - произвольная последовательность целых аналитических функций на $\mathbb{C}^{N}$, удовлетворяюших условию (4.2).

ОПРеДЕЛЕНИЕ. Свяжем с набором $u=\left(u_{1}, \ldots, u_{N}\right)$ набор $\partial_{u}=\left(\partial_{1}, \ldots, \partial_{N}\right)$ линейных операторов в пространстве аналитических функций на $\mathbb{C}^{N}$, определенных равенством:

$$
\partial_{j}=\partial_{u_{j}, j}=a_{j}^{-1} \circ \delta_{u_{j}} ; \quad j=1, \ldots, N .
$$

Таким образом, операторы $\partial_{j}$ действуют на мономы $a^{\alpha}=a_{1}^{\alpha_{1}} \cdots a_{N}^{\alpha_{N}}$ по формуле:

$$
\partial_{j} a^{\alpha}=u_{j}(\alpha) a^{\alpha-e_{j}}, \quad j=1, \ldots, N .
$$

Очевидно, что условие (4.2) на функции $u_{j}$ эквивалентно условию перестановочности операторов $\partial_{j}$.

Заметим, что если для каждого $j=1, \ldots, N$ функция $u_{j}$ зависит только от $a_{j}$, то операторы $\partial_{j}$ совпадают с введенными в $\S 3$ операторами на $\mathbb{C}$, действуюшими только на свое переменное $a_{j}$.

ПРЕДЛОЖЕНИЕ 4.4. Для любого вектора $l=\left(l_{1}, \ldots, l_{N}\right)$ с цельци неотрицательныцми координатами имеем:

$$
\prod_{j=1}^{N} \partial_{j}{ }^{l_{j}}=a^{-l} \circ \delta_{v},
$$

əде $v(\alpha)=[u(\alpha)]_{l}$.

В самом деле, легко убедиться, что $\left(\prod_{j=1}^{N} \partial_{j}^{l_{j}}\right) a^{\alpha}=a^{-l} \delta_{v} a^{\alpha}$ для любого монома $a^{\alpha}$. Отсюда следует утверждение. 
4.5. $u$-экспоненты. Пусть $\partial_{j}=\partial_{u_{j}, j}$ - операторы, определенные в п. 4.4.

ОпРеДЕлЕнИЕ. Назовем $u$-экспонентами функции на $\mathbb{C}^{N}$, удовлетворяюшие следующей системе уравнений:

$$
\partial_{j} f=f, \quad j=1, \ldots, N .
$$

Очевидно, что для любого $\lambda \in \mathbb{C}^{N}$ формальный ряд

$$
\sum_{k \in \mathbb{Z}^{N}} \frac{a^{k+\lambda}}{E_{u}(k+\lambda)}
$$

формально удовлетворяет системе (4.13). Следовательно, при условии сходимости он задает $u$-экспоненту. В частности, $u$-экспоненциальньй ряд

$$
\exp _{u} a=\sum_{k \in \mathbb{Z}^{N}} \frac{a^{k}}{E_{u}(k)}
$$

задает $u$-экспоненту при условии, что он сходится.

ЗАмечание. Если для каждого $j=1, \ldots, N$ функция $u_{j}$ зависит только от $a_{j}$, то уравнениям (4.13) удовлетворяет функция $f(a)=\prod_{j=1}^{N} f_{u_{j}}\left(a_{j}\right)$, где $f_{u_{j}}-u_{j}$-экспоненты, введенные в $\S 3$, т.е. решения уравнения $\partial_{u_{j}} f_{u_{j}}=f_{u_{j}}$.

4.6. Примеры. $1^{\circ} \cdot u_{j}(\alpha)=\alpha_{1}+\cdots+\alpha_{N}, j=1, \ldots, N$. В этом случае $E_{u}(\alpha)=$ $\Gamma\left(\alpha_{1}+\cdots+\alpha_{N}+1\right)$,

$$
\exp _{u} a=\sum_{k \in \mathbb{Z}^{N}} \frac{a^{k}}{\Gamma\left(k_{1}+\cdots+k_{N}+1\right)}, \quad \partial_{j}=a_{j}^{-1} \sum_{i=1}^{N} a_{i} \frac{\partial}{\partial a_{i}} .
$$

Уравнения (4.13) имеют вид:

$$
\left(\sum_{i=1}^{N} a_{i} \frac{\partial}{\partial a_{i}}\right) f=a_{j} f, \quad j=1, \ldots, N
$$

Очевидно, что ненулевых решений эти уравнения не имеют.

$$
\begin{aligned}
2^{\circ} . u_{j}(\alpha)=\left(\alpha_{1}+\cdots+\alpha_{N}\right) \alpha_{j}, j=1, \ldots, N . \text { В этом случае } \\
E_{u}(\alpha)=\Gamma\left(\alpha_{1}+\cdots+\alpha_{N}+1\right) \prod_{j=1}^{N} \Gamma\left(\alpha_{j}+1\right), \\
\exp _{u} a=\sum_{k_{1}, \ldots, k_{N}=0}^{\infty} \frac{a^{k}}{k_{1} ! \cdots k_{N} !\left(k_{1}+\cdots+k_{N}\right) !}, \\
\partial_{j}=\frac{\partial}{\partial a_{j}} \sum_{i=1}^{N} a_{i} \frac{\partial}{\partial a_{j}}, \quad j=1, \ldots, N .
\end{aligned}
$$


Ряд $\exp _{u} a$ сходится при любом $a \in \mathbb{C}^{N}$ и задает одно из решений системы (4.13).

$3^{\circ} \cdot u_{j}(\alpha)=q^{-\alpha_{1}-\cdots-\alpha_{N}}, j=1, \ldots, N$. В этом случае $E_{u}(\alpha)=q^{-\beta(\beta+1) / 2}$, где $\beta=\sum \alpha_{j}$

$$
\exp _{u} a=\sum_{k \in \mathbb{Z}^{N}} q^{-\left(\sum k_{i}\right)\left(\sum k_{i}+1\right) / 2} a^{k}
$$

$\left(\partial_{u_{j}} f\right)(a)=a_{j}^{-1} f\left(q^{-1} a\right)$. Уравнения (4.13) имеют вид:

$$
f\left(q^{-1} a\right)=a_{j} f(a), \quad j=1, \ldots, N .
$$

Очевидно, что ненулевых решений эти уравнения не имеют.

$$
\begin{gathered}
4^{\circ} . u_{j}(\alpha)=\frac{1-q^{a_{j}}}{1-q} q^{-\alpha_{1}-\cdots-\alpha_{N}}, j=1, \ldots, N, \text { где }|q|<1 . \text { В этом случае } \\
E_{u}(\alpha)=q^{-\left(\sum k_{i}\right)\left(\sum k_{i}+1\right) / 2} a^{k} \prod_{j=1}^{N} \Gamma_{q}\left(\alpha_{j}+1\right)
\end{gathered}
$$

(определение функции $\Gamma_{q}$ см. в $\S 1$ ),

$$
\exp _{u} a=\sum_{k_{1}, \ldots, k_{N}=0}^{\infty}\left(q^{-\left(\sum k_{i}\right)\left(\sum k_{i}+1\right) / 2} \prod_{i=1}^{N} \frac{a_{i} k_{i}}{\Gamma_{q}\left(k_{i}+1\right)}\right),
$$

$\left(\partial_{j} f\right)(a)=a_{j}^{-1}(1-q)^{-1}\left(f\left(q^{-1} a_{1}, \ldots, q^{-1} a_{N}\right)-f\left(q^{-1} a_{1}, \ldots, a_{j}, \ldots, q^{-1} a_{N}\right)\right)$. Система уравнений (4.13) приводится к виду:

$$
f\left(a_{1}, \ldots, a_{N}\right)=f\left(a_{1}, \ldots, q a_{j}, \ldots, a_{N}\right)=q(1-q) a_{j} f(q a), \quad j=1, \ldots N .
$$

Ряд $\exp _{u} a$ сходится при всех $a \in \mathbb{C}^{N}$ и задает одно из решений этой системы.

\section{$\S$ 5. Ряды гипергеометрического типа}

5.1. Случай одной переменной. Пусть $u(\alpha), \alpha \in \mathbb{C},-$ произвольная аналитическая функция, $\Gamma_{u}(\alpha)$ - ассоциированная с ней $u$-гамма-функция.

ОПРЕДЕЛЕНИЕ. Назовем рядами гипергеометрического типа, связанньми с $u$ (по-другому: Г-рядами), следуюшие формальные ряды от $x \in \mathbb{C}$ :

$$
f_{k}(\lambda, x)=\sum_{n \in \mathbb{Z}} \frac{x^{k n+\lambda}}{\Gamma_{u}(k n+\lambda+1)}=\sum_{n \in \mathbb{Z}} \frac{x^{k n+\lambda}}{E_{u}(k n+\lambda)}, \quad k \in \mathbb{Z}, \quad \lambda \in \mathbb{C} .
$$

В частности, при $k=1, \lambda \in \mathbb{Z}$ ряд (5.1) совпадает с $u$-экспоненциальньм рядом $\exp _{u} x$.

Очевидно, что Г-ряды обладают следующими свойствами:

$1^{\circ} \cdot f_{k}(\lambda, x)=f_{-k}(\lambda, x)$

$2^{\circ} \cdot f_{k}(\lambda+k, x)=f_{k}(\lambda, x)$,

$3^{\circ} \cdot f_{k}(\lambda, x)=\sum_{s=0}^{l-1} f_{k l}(\lambda+s, x)$ для любого $l=1,2, \ldots$ 
ПРЕДЛОЖЕНИЕ 5.1. Между Г-рядами и и-экспоненциальньми рядами имеется следующая связь:

$$
f_{k}(\lambda, x)=x^{\lambda} \exp _{v} y=x^{\lambda} \sum_{n \in \mathbb{Z}} \frac{y^{n}}{\Gamma_{v}(n+1)},
$$

əде $y=x^{k}, v(\alpha)=[u(k \alpha+\lambda)]_{k}$, m.e.

$$
\begin{aligned}
& v(\alpha)=\prod_{i=0}^{k-1} u(k \alpha+\lambda-i) \quad \text { npu } \quad k>0, \\
& v(\alpha)=\left(\prod_{i=1}^{|k|} u(k \alpha+\lambda+i)\right)^{-1} \quad \text { npu } \quad k<0 .
\end{aligned}
$$

ДокАЗАТЕЛЬСТво. Очевидно, что ряд $f_{k}(\lambda, x)$ представим в виде $(5.2)$, где функция $v(\alpha)$ определяется из условия $\Gamma_{v}(\alpha+1)=\Gamma_{u}(k \alpha+\lambda+1)$. Значит,

$$
v(\alpha)=\frac{\Gamma_{v}(\alpha+1)}{\Gamma_{v}(\alpha)}=\frac{\Gamma_{u}(k \alpha+\lambda+1)}{\Gamma_{u}(k \alpha+\lambda+1-k)} .
$$

Отсюда следуют выражения (5.3) для $v(\alpha)$.

ПримеР. $u(\alpha)=\frac{r^{\alpha}-s^{\alpha}}{r-s}$, где $0<|r|<|s|$. В этом случае

$$
f_{1}(\lambda, x)=x^{\lambda} \sum_{n \in \mathbb{Z}} \frac{x^{n+\lambda}}{\Gamma_{r, s}(n+\lambda+1)},
$$

где (см. п. 2.3)

$$
\Gamma_{r, s}(\alpha)=s^{(\alpha-1)(\alpha-2) / 2}(1-q)^{1-\alpha} \prod_{n=0}^{\infty} \frac{1-q^{1+n}}{1-q^{\alpha+n}}, \quad q=\frac{r}{s} .
$$

Согласно п. 3.3 этот ряд имеет непустую область сходимости тогда и только тогда, когда вьполняется одно из следующих условий:

1) $r \geqslant 1$,

2) $s \geqslant 1$ и $\lambda=\frac{2 \pi i k^{\prime}}{\ln q}+k^{\prime \prime}$, где $k^{\prime} \in \mathbb{Z}, k^{\prime \prime}=0,-1,-2, \ldots$

Отметим, что в случае 2 ) ряд обрывается при $n \rightarrow-\infty$.

5.2. Многомерные ряды гипергеометрического типа. Фиксируем произвольную последовательность $u=\left(u_{1}, \ldots, u_{N}\right)$ аналитических функций на $\mathbb{C}^{N}$, удовлетворяюших соотношениям:

$$
u_{i}(\alpha) u_{j}\left(\alpha-e_{i}\right)=u_{j}(\alpha) u_{i}\left(\alpha-e_{j}\right), \quad i, j=1, \ldots, N .
$$

Обозначим, в соответствии с п. 4.1 , через $E_{u}(\alpha)$ решение системы уравнений:

$$
E_{u}(\alpha)=u_{i}(\alpha) E_{u}\left(\alpha-e_{i}\right), \quad i=1, \ldots, N
$$

Пусть $\Lambda \subset \mathbb{Z}^{N}$ - произвольная подрешетка, т.е. подгруппа аддитивной групшы $\mathbb{Z}^{N}$. 
ОПРеДЕЛЕНИЕ. Назовем рядом гипергеометрического типа (по-другому: Г-рядом), ассоциированным с подрешеткой $\Lambda \subset \mathbb{Z}^{N}$ и вектором $\lambda \in \mathbb{C}^{N}$, следуюший формальный ряд:

$$
f_{\Lambda}(\lambda, a)=\sum_{l \in \Lambda} \frac{a^{l+\lambda}}{E_{u}(l+\lambda)}, \quad a \in \mathbb{C}^{N}
$$

где $a^{l+\lambda}=a_{1}^{l_{1}+\lambda_{1}} \cdots a_{N}^{l_{N}+\lambda_{N}}$.

В частности, при $\Lambda=\mathbb{Z}^{N}$ имеем:

$$
f_{\mathbb{Z}^{N}}(\lambda, a)=a^{\lambda} \exp _{v} a,
$$

где $v(\alpha)=u(\alpha+\lambda)$, a $\exp _{v} a-v$-экспоненщиальный ряд.

Очевидно, что ряды $f_{\Lambda}(\lambda, a)$ обладают следуюшими свойствами:

$1^{\circ} . f_{\Lambda}(\lambda+l, a)=f_{\Lambda}(\lambda, a)$ для любого $l \in \Lambda$,

$2^{\circ}$. если $\Lambda_{1} \subset \Lambda_{2}$, то $f_{\Lambda_{2}}(\lambda, a)=\sum_{l \in \Lambda_{2} / \Lambda_{1}} f_{\Lambda_{1}}(\lambda+l, a)$.

В частности,

$$
a^{\lambda} \exp _{v} a=\sum_{l \in \mathbb{Z}^{N} / \Lambda} f_{\Lambda}(\lambda+l, a), \quad v(\alpha)=u(\alpha+\lambda)
$$

для любой подрешетки $\Lambda \subset \mathbb{Z}^{N}$. Из (5.6) следует

ПрЕДЛОЖЕНИЕ 5.2. Если при $\lambda$ общего положения ряд $\exp _{v}$ a сходится в некоторой области $U \subset \mathbb{C}^{N}$, то в этой области сходится и ряд $f_{\Lambda}(\lambda, a)$, әде $\Lambda \subset \mathbb{Z}^{N}$ - любая подрешетка.

5.3. Приведенные ряды гипергеометрического типа. Фиксируем произвольный базис в подрешетке $\Lambda \subset \mathbb{Z}^{N}$ :

$$
l^{i}=\left(l_{1}^{i}, \ldots, l_{N}^{i}\right), \quad i=1, \ldots, n ;
$$

$n=\operatorname{rank} \Lambda$. Таким образом,

$$
\Lambda=\left\{k_{1} l^{1}+\cdots+k_{n} l^{n} \mid k \in \mathbb{Z}^{n}\right\} .
$$

Из (5.5) непосредственно следует

ПРЕДЛОЖЕНИЕ 5.3. Любой Г-ряд $f_{\Lambda}(\lambda, a)$ представим в виде

$$
f_{\Lambda}(\lambda, a)=a^{\lambda} \sum_{k \in \mathbb{Z}^{n}} \frac{x^{k}}{E_{u}\left(k_{1} l^{1}+\cdots+k_{n} l^{n}+\lambda\right)}, \quad x=\left(x_{1}, \ldots, x_{n}\right),
$$

əде $x^{k}=x_{1}^{k_{1}} \cdots x_{n}^{k_{n}}$

$$
x_{i}=a^{l^{i}}=a_{1}^{l_{1}^{i}} \cdots a_{N}^{l_{N}^{i}} .
$$


ОПРЕДЕЛЕНИЕ. Назовем формальньй ряд

$$
F_{\Lambda}(\lambda, x)=\sum_{k \in \mathbb{Z}^{n}} \frac{x^{k}}{E_{u}\left(k_{1} l^{1}+\cdots+k_{n} l^{n}+\lambda\right)}, \quad x \in \mathbb{C}^{n}
$$

приведенным рядом гипергеометрического типа (по другому: приведенным Г-рядом), ассоциированным с подрешеткой $\Lambda \subset \mathbb{Z}^{N}$ и вектором $\lambda \in \mathbb{C}^{N}$.

Подчеркнем, что этот ряд зависит и от выбора базиса $l^{1}, \ldots, l^{n}$ в $\Lambda$.

В силу предложения 5.3 Г-ряд $f_{\Lambda}(\lambda, a)$ и приведенный $\Gamma$-ряд $F_{\Lambda}(\lambda, x)$ связаны соотношением:

$$
f_{\Lambda}(\lambda, a)=a^{\lambda} F_{\Lambda}(\lambda, x)
$$

где $x \in \mathbb{C}^{n}$ выражается через $a \in \mathbb{C}^{N}$ по формулам (5.8).

ПРЕДЛОЖЕНИЕ 5.4. Приведенные Г-ряды являются рядами әкспоненииально20 muna:

$$
F_{\Lambda}(\lambda, x)=\exp _{v} x=\sum_{k \in \mathbb{Z}^{n}} \frac{x^{k}}{E_{v}(k)},
$$

где последовательность $v=\left(v_{1}, \ldots, v_{N}\right)$ функиий на $\mathbb{C}^{n}$ определяется из соотношений

$$
v_{i}(\beta)=\left[u\left(\beta_{1} l^{1}+\cdots+\beta_{n} l^{n}+\lambda\right)\right]_{l^{i}}
$$

(определение символа $[u(\alpha)]_{l}$ см. в п. 4.3).

ДокАЗАТЕЛЬСТВо. Положив

$$
E_{v}(\beta)=E_{u}\left(\beta_{1} l^{1}+\cdots+\beta_{n} l^{n}+\lambda\right),
$$

получаем для функций $v_{i}$ в последовательности $v=\left(v_{1}, \ldots, v_{N}\right)$ следующие выражения:

$$
v_{i}(\beta)=\frac{E_{v}(\beta)}{E_{v}\left(\beta-e_{i}\right)}=\frac{E_{u}\left(\beta_{1} l^{1}+\cdots+\beta_{n} l^{n}+\lambda\right)}{E_{u}\left(\beta_{1} l^{1}+\cdots+\beta_{n} l^{n}+\lambda-l^{i}\right)}=\left[u\left(\beta_{1} l^{1}+\cdots+\beta_{n} l^{n}+\lambda\right)\right]_{l^{i}} .
$$

Рассмотрим случай, когда каждая функция $u_{j}$ зависит только от $\alpha_{j}$. В этом случае

$$
E_{u}(\alpha)=\Gamma_{u_{1}}\left(\alpha_{1}+1\right) \cdots \Gamma_{u_{N}}\left(\alpha_{N}+1\right),
$$

где $\Gamma_{u_{i}}$ - гамма-функции одного переменного, а потому приведенньй Г-ряд имеет вид:

$$
F_{\Lambda}(\lambda, a)=\sum_{k \in \mathbb{Z}^{n}} \frac{x^{k}}{\Gamma_{u_{1}}\left(\mu_{1}(k)+\lambda_{1}+1\right) \cdots \Gamma_{u_{N}}\left(\mu_{N}(k)+\lambda_{N}+1\right)},
$$


где

$$
\mu_{j}(k)=k_{1} l_{j}^{1}+\cdots+k_{n} l_{j}^{n}, \quad j=1, \ldots, N
$$

В частности,

$$
F_{\mathbb{Z}^{N}}(\lambda, x)=\prod_{i=1}^{N}\left(\sum_{k \in \mathbb{Z}} \frac{x_{i}{ }^{k+\lambda_{i}}}{\Gamma_{u_{i}}\left(k+\lambda_{i}+1\right)}\right) .
$$

Выражения (5.11) для функций $v_{i}(\beta)$ сводятся в этом случае к следуюшим:

$$
v_{i}(\beta)=\prod_{j=1}^{N} v_{i, j}(\beta)
$$

где $v_{i, j}(\beta)=1$, если $l_{j}^{i}=0$,

$$
\begin{aligned}
& v_{i, j}(\beta)=\prod_{s=0}^{l_{j}^{i}-1} u_{j}\left(\mu_{j}(\beta)+\lambda_{j}-s\right), \quad \text { если } l_{j}^{i}>0, \\
& v_{i, j}(\beta)=\left(\prod_{s=1}^{\left|l_{j}^{i}\right|} u_{j}\left(\mu_{j}(\beta)+\lambda_{j}+s\right)\right)^{-1}, \text { если } l_{j}^{i}<0 .
\end{aligned}
$$

5.4. Замечание о сходимости Г-рядов. В “классическом" случае, когда все $\Gamma_{u_{i}}$ суть гамма-функции Эйлера, условия сходимости рядов (5.13) были подробно исследованы, см., например, [5]. Было установлено, что любой Г-ряд с непустой областью сходимости обрьвается при $k_{i} \rightarrow-\infty$ или $k_{i} \rightarrow+\infty, i=1, \ldots, N$. Установлено, что если $L \subset \mathbb{C}^{N}$ - линейное подпространство, порожденное подрешеткой $\Lambda$, и $\alpha \in \mathbb{C}^{N}$ - произвольный фиксированный вектор, то среди рядов $f_{\Lambda}(\lambda, a)$, где $\lambda \in \alpha+L$, лишш конечное число рядов имеет непустую область сходимости.

В общем случае эти факты, вообще говоря, не имеют места. Рассмотрим, например, случай, когда $u_{i}(\alpha)=q_{i}^{-\alpha}, 0<\left|q_{i}\right|<1, i=1, \ldots, N$. В этом случае $\Gamma_{u_{i}}(\alpha)=$ $q_{i}^{-\alpha(\alpha-1) / 2}$, а потому

$$
\sum_{k \in \mathbb{Z}} \frac{a_{i}{ }^{k+\lambda_{i}}}{\Gamma_{u_{i}}\left(k+\lambda_{i}+1\right)}=\sum_{k \in \mathbb{Z}} q_{i}^{\left(k+\lambda_{i}\right)\left(k+\lambda_{i}+1\right) / 2} a_{i}^{k+\lambda_{i}} .
$$

Очевидно, что при любом $\lambda_{i}$ этот ряд сходится для всех $a_{i} \neq 0$. Отсюда следует, что в приведенном примере ряды (5.13) нигде не обрьваются и сходятся для каждого $a \in \mathbb{C}^{N}$ с отличньми от нуля координатами.

5.5. Г-ряды, ассоциированные с подрешетками $\Lambda$ ранга 1 . Рассмотрим случай, когда подрешетка $\Lambda \subset \mathbb{Z}^{N}$ порождена одним вектором $l=\left(l_{1}, \ldots, l_{n}\right) \in \mathbb{Z}^{N}$, т.е. $\Lambda=\{n l \mid n \in \mathbb{Z}\}$. В этом случае приведенньй Г-ряд, ассоциированньй с $\Lambda$ и вектором $\lambda \in \mathbb{C}^{N}$, имеет вид:

$$
F_{\Lambda}(\lambda, x)=\exp _{v} x=\sum_{k \in \mathbb{Z}} \frac{x^{k}}{\Gamma_{v}(k+1)}, \quad x \in \mathbb{C},
$$


где $v(\beta)$ - функция одного переменного, заданная равенством

$$
v(\beta)=[u(\beta l+\lambda)]_{l} .
$$

Если каждая из функций $u_{i}(\alpha)$ зависит только от $\alpha_{i}, i=1, \ldots, N$, то

$$
F_{\Lambda}(\lambda, x)=\sum_{k \in \mathbb{Z}} \frac{x^{k}}{\Gamma_{u_{1}}\left(k l_{1}+\lambda_{1}+1\right) \cdots \Gamma_{u_{N}}\left(k l_{N}+\lambda_{N}+1\right)},
$$

а функции $v(\beta)$ имеют вид:

$$
v(\beta)=\prod_{j=1}^{N} v_{j}(\beta),
$$

где

$$
v_{j}(\beta)= \begin{cases}1 & \text { при } l_{j}=0, \\ \prod_{s=0}^{l_{j}-1} u_{j}\left(\beta l+\lambda_{j}-s\right) & \text { при } l_{j}>0, \\ \left(\prod_{s=1}^{\left|l_{j}\right|} u_{j}\left(\beta l+\lambda_{j}+s\right)\right)^{-1} & \text { при } l_{i}<0 .\end{cases}
$$

Приведем критерий сходимости рядов (5.16).

Не нарушая обшности, можно считать, что все координаты вектора $l$ отличны от нуля. Предположим, что функщии $\Gamma_{u_{i}}$ не имеют нулей и обладают следуюшей асимптотикой:

$$
\begin{aligned}
& \Gamma_{u_{i}}(\alpha+n+1) \sim c_{i}^{\prime} \sigma_{i}^{n} \mu_{i}^{n^{2}} \text { при } n \rightarrow+\infty, \\
& \Gamma_{u_{i}}(\alpha+n+1) \sim c_{i}^{\prime \prime} \tau_{i}^{n} \nu_{i}^{n^{2}} \text { при } n \rightarrow-\infty,
\end{aligned}
$$

где $c_{i}^{\prime}, c_{i}^{\prime \prime}, \sigma_{i}, \tau_{i}, \mu_{i}, \nu_{i}$ зависят, вообще говоря, от $\alpha$. Этим свойством обладают, например, функции $\Gamma_{u_{i}}=\Gamma_{u_{i}}^{+}$, отвечающие $u_{i}(\alpha)=\sum_{j=1}^{p} \gamma_{i j} r_{i j}{ }^{\alpha}$, в частности, отвечающие $u_{i}(\alpha)=\frac{r_{i}^{\alpha}-s_{i}^{\alpha}}{r_{i}-s_{i}}$. Так, если $u_{i}(\alpha)=\frac{r_{i}^{\alpha}-s_{i}^{\alpha}}{r_{i}-s_{i}}$, где $0<\left|r_{i}\right|<\left|s_{i}\right|, i=1, \ldots, N$, то $\Gamma_{u_{i}}=\Gamma_{r_{i}, s_{i}}$ (см. п. 1.6) и, на основании п. 1.6, имеем следуюшие выражения для $\sigma_{i}$, $\tau_{i}, \mu_{i}, \nu_{i}$ в $(5.17)$ :

$$
\sigma_{i}=\left(s_{i}-r_{i}\right)^{-1} s_{i}^{\alpha-1 / 2}, \quad \tau_{i}=\left(r_{i}-s_{i}\right)^{-1} s_{i}^{\alpha-1 / 2}, \quad \mu_{i}=s_{i}^{1 / 2}, \quad \nu_{i}=r_{i}^{1 / 2} .
$$

Из (5.17) вытекают следуюшие оценки для коэффициентов $c(k)$ ряда $(5.16)$ :

$$
\begin{gathered}
c(k) \sim c^{\prime} \sigma_{+}^{-k} \rho_{+}^{-k^{2}} \text { при } k \rightarrow+\infty, \\
c(k) \sim c^{\prime \prime} \sigma_{-}^{-k} \rho_{-}^{-k^{2}} \text { при } k \rightarrow-\infty,
\end{gathered}
$$

где

$$
\begin{array}{ll}
\sigma_{+}=\prod_{l_{i}>0} \sigma_{i}{ }^{l_{i}} \prod_{l_{i}<0} \tau_{i}{ }^{l_{i}}, & \sigma_{-}=\prod_{l_{i}<0} \sigma_{i}{ }^{{ }{ }_{i}} \prod_{l_{i}>0} \tau_{i}{ }^{l_{i}}, \\
\rho_{+}=\prod_{l_{i}>0} \mu_{i}{ }^{{ }^{2}{ }^{2}} \prod_{l_{i}<0} \nu_{i}{ }^{l_{i}{ }^{2}}, & \rho_{-}=\prod_{l_{i}<0} \mu_{i}{ }^{{ }^{2}{ }^{2}} \prod_{l_{i}>0} \nu_{i}{ }^{{ }{ }^{2}{ }^{2} .}
\end{array}
$$


Из этих оценок непосредственно вытекают следующие критерии сходимости ряда $(5.16)$.

$1^{\circ}$. Если ряд обрьвается при $k \rightarrow-\infty$, то в случае $\left|\rho_{+}\right|>1$ он сходится для всех $x \neq 0$; в случае $\left|\rho_{+}\right|=1$ сходится в области $0<|x|<\left|a_{+}\right| ;$при $\left|\rho_{+}\right|<1$ ряд всюду расходится. ${ }^{2}$

$2^{\circ}$. Если ряд обрьвается при $k \rightarrow+\infty$, то в случае $\left|\rho_{-}\right|>1$ он сходится для всех $x \neq 0$; в случае $\left|\rho_{-}\right|=1$ сходится в области $|x|>\left|a_{-}\right|$; при $\left|\rho_{-}\right|<1$ ряд всюду расходится.

$3^{\circ}$. Если ряд не обрьвается ни при $k \rightarrow-\infty$, ни при $k \rightarrow+\infty$, то возможны следующие варианты:

\begin{tabular}{|c|c|}
\hline Условия на $\rho_{ \pm}$и $a_{ \pm}$ & Область сходимости \\
\hline$\left|\rho_{+}\right|>1,\left|\rho_{-}\right|>1$ & $x \neq 0$ \\
\hline$\left|\rho_{+}\right|=1,\left|\rho_{-}\right|>1$ & $0<|x|<\left|\sigma_{+}\right|$ \\
\hline$\left|\rho_{+}\right|>1,\left|\rho_{-}\right|=1$ & $|x|>\left|\sigma_{-}\right|$ \\
\hline$\left|\rho_{+}\right|=\left|\rho_{-}\right|=1,\left|\sigma_{-}\right|<\left|\sigma_{+}\right|$ & $\left|\sigma_{-}\right|<|x|<\left|\sigma_{+}\right|$ \\
\hline$\left|\rho_{+}\right|=\left|\rho_{-}\right|=1,\left|\sigma_{-}\right| \geqslant\left|\sigma_{+}\right|$ & всюду расходится \\
\hline$\left|\rho_{+}\right|<1$ или $\left|\rho_{-}\right|<1$ & всюду расходится \\
\hline
\end{tabular}

5.6. $u$-аналоги гипергеометрического ряда Гаусса. Пусть $u=\left(u_{1}, u_{2}, u_{3}, u_{4}\right)$ - произвольная последовательность аналитических функций на $\mathbb{C}^{4}$, где $u_{i}$ зависит только от одного аргумента $\alpha_{i}$, и пусть $\Lambda \subset \mathbb{Z}^{4}$ - подрешетка ранга 1 , порожденная вектором $l=(-1,-1,1,1)$.

ОПРЕДЕЛЕНИЕ. Назовем $u$-аналогами гипергеометрического ряда Гаусса приведенные Г-ряды, ассоциированные с $\Lambda$ и произвольным вектором $\lambda \in \mathbb{C}^{4}$, т.е. ряды вида

$F(\lambda, x)=\sum_{k=-\infty}^{+\infty} \frac{x^{k}}{\Gamma_{u_{1}}\left(-k+\lambda_{1}+1\right) \Gamma_{u_{2}}\left(-k+\lambda_{2}+1\right) \Gamma_{u_{3}}\left(-k+\lambda_{3}+1\right) \Gamma_{u_{4}}\left(-k+\lambda_{4}+1\right)}$.

ПРЕДЛОЖЕНИЕ 5.5. С точностью до множителя, зависящего только от $\lambda_{1}$,

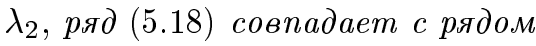

$$
F(\lambda, x)=\sum_{k=-\infty}^{+\infty} \frac{\Gamma_{v_{1}}\left(k-\lambda_{1}\right) \Gamma_{v_{2}}\left(k-\lambda_{2}\right)}{\Gamma_{u_{3}}\left(k+\lambda_{3}+1\right) \Gamma_{u_{4}}\left(k+\lambda_{4}+1\right)} x^{k}
$$

əде $v_{i}(\alpha)=u_{i}(-\alpha), i=1,2$.

\footnotetext{
${ }^{2}$ Мы исключаем из рассмотрения случай, когда ряд обрывается и при $k \rightarrow-\infty$, и при $k \rightarrow+\infty$, и тем самым сводится к лорановскому полиному.
} 
В самом деле, согласно п. $1.2 \Gamma_{u_{i}}(\alpha)=\frac{\theta_{i}(\alpha)}{\Gamma_{v_{i}}(1-\alpha)}$, где $\theta_{i}(\alpha)$-периодическая функция с периодом 1, и, значит,

$$
\Gamma_{u_{i}}\left(-k+\lambda_{i}+1\right)=\frac{\theta_{i}\left(\lambda_{i}\right)}{\Gamma_{v_{i}}\left(k-\lambda_{i}\right)} .
$$

Отсюда следует утверждение.

Классический ряд Гаусса соответствует случаю, когда $\Gamma_{u_{i}}$ суть гамма-функции Эйлера, $i=1,2,3,4$, и одно из чисел $\lambda_{3}, \lambda_{4}$ равно 0.

ЗАмечАниЕ. При произвольном $N$ естественно назвать $\Gamma$-ряды, ассоциированные с подрешеткой $\Lambda \subset \mathbb{Z}^{N}$ ранга 1 , порожденной вектором $l=\left(\varepsilon_{1}, \ldots, \varepsilon_{N}\right)$, где $\varepsilon_{i}= \pm 1$, $u$-аналогами гипергеометрического ряда Похгаммера.

Рассмотрим случай $u_{i}(\alpha)=\frac{r_{i}^{\alpha}-s_{i}^{\alpha}}{r_{i}-s_{i}}$, где $0<\left|r_{i}\right|<\left|s_{i}\right|, i=1,2,3,4$. В этом случае условия сходимости ряда (5.18) следуют из критерия, приведенного в п. 5.4; достаточно заметить, что в обозначениях п. 5.5 мы имеем:

$$
\begin{array}{ll}
\rho_{+}=\left(r_{1} r_{2} s_{3} s_{4}\right)^{1 / 2}, & \rho_{-}=\left(s_{1} s_{2} r_{3} r_{4}\right)^{1 / 2}, \\
\sigma_{+}=c \rho_{+}^{2 \alpha-1}, & \sigma_{-}=c^{-1} \rho_{-}^{2 \alpha-1},
\end{array}
$$

где $c=\frac{\left(s_{1}-r_{1}\right)\left(s_{2}-r_{2}\right)}{\left(s_{3}-r_{3}\right)\left(s_{4}-r_{4}\right)}$. Согласно приведенному критерию ряд $F_{\Lambda}(\lambda, x)$ при любом $\lambda$ имеет непустую область сходимости, если $\left|\rho_{+}\right| \geqslant 1,\left|\rho_{-}\right| \geqslant 1$ и $\left|\rho_{+} \rho_{-}\right| \neq 1$. Если же $\left|\rho_{+}\right|<1,\left|\rho_{-}\right|<1$, то ряд всюду расходится за исключением значений $\lambda$, при которых он сводится к лорановскому полиному.

В частности, если $s_{i}=1, r_{i}=q_{i}, 0<\left|q_{i}\right|<1, i=1,2,3,4$, то сходяшихся $Г$-рядов не сушествует.

\section{§. Общие $u$-гипергеометрические системы}

6.1. Определение общей гипергеометрической системы. Фиксируем произвольную последовательность

$$
u=\left(u_{1}, \ldots, u_{N}\right)
$$

целых аналитических функций на $\mathbb{C}^{N}$, удовлетворяюших соотношениям

$$
u_{i}(\alpha) u_{j}\left(\alpha-e_{i}\right)=u_{j}(\alpha) u_{i}\left(\alpha-e_{j}\right), \quad i, j=1, \ldots, N
$$

Пусть $\partial_{1}, \ldots, \partial_{N}$ - связанные с $u$ линейные операторы в пространстве аналитических функций на $\mathbb{C}^{N} ;$ по определению (см. $\left.\S 4\right) \partial_{j}$ действует на мономы $a^{\alpha}=a_{1}^{\alpha_{1}} \cdots a_{N}^{\alpha_{N}}$ по формуле

$$
\partial_{j} a^{\alpha}=u_{j}(\alpha) a^{\alpha-e_{j}} .
$$

Обозначим через $\Lambda$ произвольную подрешетку в $\mathbb{Z}^{N}$ и через $L \subset \mathbb{C}^{N}$ - натянутое на $\Lambda$ линейное подпространство. 
ОПРЕДЕЛЕНИЕ. Назовем общей $u$-гипергеометрической системой, ассоциированной с подрешеткой $\Lambda \subset \mathbb{Z}^{N}$ и вектором $\beta \in \mathbb{C}^{N} / L$, следующую систему уравнений в пространстве функций на $\mathbb{C}^{N}$ :

$$
\begin{gathered}
\prod_{j: l_{j}>0} \partial_{j}{ }^{l_{j}} f=\prod_{j: l_{j}<0} \partial_{j}{ }^{-l_{j} f} \text { для любого } l=\left(l_{1}, \ldots, l_{N}\right) \in \Lambda ; \\
\sum_{j=1}^{N} \omega^{j} a_{j} \frac{\partial f}{\partial a_{j}}=\langle\omega, \beta\rangle f
\end{gathered}
$$

для любого вектора $\omega=\left(\omega^{1}, \ldots, \omega^{N}\right) \in\left(\mathbb{C}^{N}\right)^{\prime}$, ортогонального $\Lambda$. Голоморфные решения этой системы назовем общими и-гипергеометрическими функииями на $\mathbb{C}^{N}$, ассочиированньми с $\Lambda$ и .

ЗАмечАниЕ. Система (6.2), (6.3) эквивалентна подсистеме из $N$ уравнений, в которой $l$ пробегает какой-либо базис в подрешетке $\Lambda$, а $\omega$ - какой-либо базис в подрешетке $\Lambda^{\perp} \subset\left(\mathbb{Z}^{N}\right)^{\prime}$, ортогональной $\Lambda$.

Система (6.2), (6.3) для случая $\partial_{j}=\frac{\partial}{\partial a_{j}}, j=1, \ldots, N$, впервые была введена в [3] и исследована в ряде других работ. Была установлена ее голономность и были построены базисы в пространстве ее решений в форме рядов гипергеометрического типа и в форме интегралов. Отметим, что все классические гипергеометрические функции здесь возникают как частные случаи обших гипергеометрических функций при специальном выборе $N$ и подрешетки $\Lambda \subset \mathbb{Z}^{N}$.

Другая важная обшая гипергеометрическая система, частные случаи которой возникают в $q$-анализе и теории квантовых групп, получается заменой операторов $\partial_{j}=$ $\frac{\partial}{\partial a_{j}}$ операторами $q$-дифференцирования по $a_{j}, j=1, \ldots, N:\left(\partial_{j} f\right)(t)=\frac{f(q t)-f(t)}{q t-t}$. Более общий случай: $\left(\partial_{j} f\right)(t)=\frac{f(r t)-f(s t)}{r t-s t}$.

Представим уравнения (6.2) в другой, эквивалентной, форме. Для этого вначале представим векторы $l \in \Lambda$ в виде разностей векторов с целыми неотрицательными координатами:

$$
l=l^{+}-l^{-},
$$

где $l_{j}^{+}=\frac{1}{2}\left(\left|l_{j}\right|+l_{j}\right), l_{j}^{-}=\frac{1}{2}\left(\left|l_{j}\right|-l_{j}\right), j=1, \ldots, N$. В этих обозначениях система $(6.2)$ принимает вид:

$$
\prod_{j=1}^{N} \partial_{j}{ }_{j}^{+} f=\prod_{j=1}^{N} \partial_{j}{ }^{l_{j}^{-}} f \quad \text { для любого } l=\left(l_{1}, \ldots, l_{N}\right) \in \Lambda .
$$

ПРЕДЛОЖЕНИЕ 6.1. Система уравнений (6.4) эквивалентна следующей:

$$
\delta_{v_{l}^{+}} f=a^{l} \delta_{v_{l}^{-}} f
$$

$2 \partial e$

$$
v_{l}^{+}(\alpha)=[u(\alpha)]_{l^{+}}, \quad v_{l}^{-}(\alpha)=[u(\alpha)]_{l^{-}},
$$

$\delta_{v}$ - операторьи, введеннье в $\S 4$. 
В самом деле, согласно (4.12),

$$
\prod_{j=1}^{N} \partial_{j}{ }_{j}^{+}=a^{-l^{+}} \delta_{v_{l}^{+}}, \quad \prod_{j=1}^{N} \partial_{j}{ }^{l_{j}^{-}}=a^{-l^{-}} \delta_{v_{l}^{-}} .
$$

Отсюда следует (6.5).

6.2. Приведенные $u$-гипергеометрические системы. Рассмотрим общую $u$-гипергеометрическую систему (6.2), (6.3) ассоциированную с подрешеткой $\Lambda \subset \mathbb{Z}^{N}$ и вектором $\beta \in \mathbb{C}^{N} / L$. Фиксируем произвольньй вектор $\lambda \in \mathbb{C}^{N}$ в прообразе вектора $\beta$ и произвольный базис

$$
l^{i}=\left(l_{1}^{i}, \ldots, l_{N}^{i}\right), \quad i=1, \ldots, n,
$$

в подрешетке $\Lambda$. Положим

$$
x_{i}=a^{l^{i}}=a_{1}^{l_{1}^{i}} \cdots a_{N}^{l_{N}^{i}}, \quad i=1, \ldots, n
$$

ПРЕДЛОЖЕНИЕ 6.2. Любое решение $f(a)$ системь (6.2), (6.3) представимо в виде:

$$
f(a)=a^{\lambda} F(x), \quad x=\left(x_{1}, \ldots, x_{n}\right) .
$$

ДоКАЗАТЕЛЬСТВо. Положим $\varphi(a)=a^{-\lambda} f(a)$ и заметим, что функция $a^{\lambda}$ удовлетворяет уравнениям (6.3). Поэтому, подставив в (6.3) выражение $f(a)=a^{\lambda} \varphi(a)$, получим:

$$
\sum_{i=1}^{N} \omega^{i} a_{i} \frac{\partial \varphi}{\partial a_{i}}=0 \text { для любого } \omega \in \Lambda^{\perp} \text {. }
$$

Отсюда следует, что $\varphi$ зависит только от $x_{i}=a^{l^{i}}, i=1, \ldots, n$, т.е. $\varphi(a)=F(x)$.

Подставив (6.7) в уравнения (6.5) $u$-гипергеометрической системы, где $l$ пробегает векторы базиса $l^{1}, \ldots, l^{n}$ в $\Lambda$, получим систему уравнений для функции $F$.

ОПРеДЕЛЕНИЕ. Назовем функции $F(x)$ на $\mathbb{C}^{n}, n=\operatorname{rank} \Lambda$, связанные с $u$-гипергеометрическими функциями $f(a)$ соотношением (6.7), приведеннымм и-гипергеометрическими функциями, ассоциированными с подрешеткой $\Lambda \subset \mathbb{Z}^{N}$ и вектором $\lambda \in \mathbb{C}^{N}$. Систему уравнений, которой эти функции удовлетворяют, назовем приведенной и-гипергеометрической системой, ассоциированной с $\Lambda$ и.

Найдем явньй вид приведенной системы.

Поставим в соответствие исходному набору $u=\left(u_{1}, \ldots, u_{N}\right)$ цельх аналитических функций на $\mathbb{C}^{N}$ два набора

$$
w^{+}=\left(w_{1}^{+}, \ldots, w_{n}^{+}\right), \quad w^{-}=\left(w_{1}^{-}, \ldots, w_{n}^{-}\right)
$$


целых аналитических функций на $\mathbb{C}^{n}$, заданных следующими равенствами:

$$
\begin{aligned}
& w_{i}^{+}(\beta)=\left[u\left(\beta_{1} l^{1}+\cdots+\beta_{n} l^{n}+\lambda\right)\right]_{\left(l^{i}\right)^{+}}, \\
& w_{i}^{-}(\beta)=\left[u\left(\beta_{1} l^{1}+\cdots+\beta_{n} l^{n}+\lambda\right)\right]_{\left(l^{i}\right)^{-}},
\end{aligned}
$$

где $i=1, \ldots, n$.

В частности, если каждая функция $u_{j}(\alpha)$ зависит только от одного аргумента $\alpha_{j}$, $j=1, \ldots, N$, то выражения (6.8) имеют вид:

$$
\begin{aligned}
& w_{i}^{+}(\beta)=\prod_{j: l_{j}^{i}>0} \prod_{s=0}^{l_{j}^{i}-1} u_{j}\left(\beta_{1} l_{j}^{1}+\cdots+\beta_{n} l_{j}^{n}+\lambda_{j}-s\right), \\
& w_{i}^{-}(\beta)=\prod_{j: l_{j}^{i}<0} \prod_{s=0}^{\left|l_{j}^{i}\right|-1} u_{j}\left(\beta_{1} l_{j}^{1}+\cdots+\beta_{n} l_{j}^{n}+\lambda_{j}-s\right) .
\end{aligned}
$$

ПРЕДЛОЖЕНИЕ 6.3. Приведенная и-гипергеометрическая система, ассоииированная с подрешеткой $\Lambda \subset \mathbb{Z}^{N}$ и вектором $\lambda \in \mathbb{C}^{N}$, имеет вид:

$$
\delta_{w_{i}^{+}} F=x_{i} \delta_{w_{i}^{-}} F, \quad i=1, \ldots, n
$$

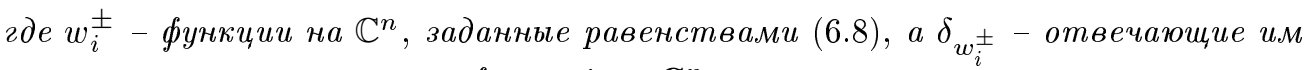
операторы в пространстве функиий на $\mathbb{C}^{n}$.

ДокАЗАТЕЛЬСТво. Подставим $f(a)=a^{\lambda} F(x)$ в уравнения (6.5), где $l$ пробегает базис $l^{1}, \ldots, l^{n}$ решетки $\Lambda$. Используя соотношение $\delta_{u}\left(a^{\lambda} F\right)=a^{\lambda} \delta_{v} F$, где $v(\alpha)=$ $u(\alpha+\lambda)$, получаем:

$$
\delta_{v_{i}^{+}} F=x_{i} \delta_{v_{i}^{-}} F, \quad i=1, \ldots, n
$$

где

$$
v_{i}^{ \pm}(\alpha)=[u(\alpha+\lambda)]_{\left(l^{i}\right)^{ \pm}},
$$

а операторы $\delta_{v_{i}^{ \pm}}$действуют на $F$ как на функщию от $a$. Убедимся, что $\delta_{w_{i}^{ \pm}} F=\delta_{v_{i}^{ \pm}} F$. Достаточно проверить это равенство для мономов $x^{\beta}$. Поскольку $x^{\beta}=a^{\beta_{1}} l^{1}+\cdots+\beta_{n} l^{n}$, то

$$
\delta_{v_{i}^{ \pm}} x^{\beta}=\left[u\left(\beta_{1} l^{1}+\cdots+\beta_{n} l^{n}+\lambda\right)\right]_{\left(l^{i}\right)^{ \pm}} x^{\beta}=\delta_{w_{i}^{ \pm}} x^{\beta}
$$

что и требовалось доказать.

Рассмотрим специальньй случай, когда целье функции $u_{j}$ не имеют нулей. В этом случае функции $w_{i}^{-}$также не имеют нулей, и, значит, функции $\left(w_{i}^{-}\right)^{-1}$ являются целыми. Поэтому равенства (6.9) эквивалентны следующим:

$$
\widetilde{\partial}_{i} F=F, \quad i=1, \ldots, n
$$


где $\widetilde{\partial}_{i}=x_{i}^{-1} \delta_{w_{i}}, w_{i}(\beta)=\left(w_{i}^{-}\left(\beta-e_{i}\right)\right)^{-1} w_{i}^{+}(\beta)$. Согласно (4.4)

$$
w_{i}(\beta)=\left[u\left(\beta_{1} l^{1}+\cdots+\beta_{n} l^{n}+\lambda\right)\right]_{l^{i}} .
$$

Из равенства (6.10) следует, что приведенная $u$-гипергеометрическая функция $F$ является $w$-экспонентой на $\mathbb{C}^{n}$, где $w=\left(w_{1}, \ldots, w_{n}\right)$.

6.3. Приведенные гипергеометрические системы, ассоциированные с решетками ранга $1 . \quad$ Если подрешетка $\Lambda \subset \mathbb{Z}^{N}$ порождена одним вектором $l=\left(l_{1}, \ldots, l_{N}\right) \in \mathbb{Z}^{N}$, то приведенные $u$-гипергеометрические функции $F$ являются функциями одного переменного, а система (6.9) состоит из одного уравнения:

$$
\delta_{w^{+}} F=x \delta_{w^{-}} F,
$$

где $w^{+}(\beta)=[u(\beta l+\lambda)]_{l^{+}}, w^{-}(\beta)=[u(\beta l+\lambda)]_{l^{-}}$.

В частности, если каждая функция $u_{j}(\alpha)$ зависит только от одного аргумента $\alpha_{j}$, то

$$
\begin{aligned}
& w_{i}^{+}(\beta)=\prod_{j: l_{j}^{i}>0} \prod_{s=0}^{l_{j}^{i}-1} u_{j}\left(\beta l_{j}+\lambda_{j}-s\right), \\
& w_{i}^{-}(\beta)=\prod_{j: l_{j}^{i}<0} \prod_{s=0}^{\left|l_{j}^{i}\right|-1} u_{j}\left(\beta l_{j}+\lambda_{j}-s\right) .
\end{aligned}
$$

Приведем явные выражения операторов $\delta_{w \pm}$ для случая, когда $u_{j}(\alpha)=\alpha_{j}$, и для случая, когда $u_{j}(\alpha)=\frac{r_{j}^{\alpha_{j}}-s_{j}^{\alpha_{j}}}{r_{j}-s_{j}}, j=1, \ldots, N$.

$1^{\circ}$. В “классическом" случае $u_{j}(\alpha)=\alpha_{j}$ функции $v_{j}(\beta)=u_{j}\left(\beta l_{j}+\lambda_{j}-s\right)=$ $\beta l_{j}+\lambda_{j}-s$ отвечает оператор $\delta_{v_{j}}=l_{j} x \frac{d}{d x}+\lambda_{j}-s$. Следовательно, приведенное гипергеометрическое уравнение имеет вид:

$$
\prod_{j: l_{j}>0} \prod_{s=0}^{l_{j}-1}\left(l_{j} x \frac{d}{d x}+\lambda_{j}-s\right) F=x \prod_{j: l_{j}<0} \prod_{s=0}^{\left|l_{j}\right|-1}\left(l_{j} x \frac{d}{d x}+\lambda_{j}-s\right) F .
$$

$2^{\circ}$. В случае $u_{j}(\alpha)=\frac{r_{j}^{\alpha_{j}}-s_{j}^{\alpha_{j}}}{r_{j}-s_{j}}, j=1, \ldots, N$, имеем:

$$
u_{j}\left(\beta l_{j}+\lambda_{j}-s\right)=\frac{r_{j}^{\beta l_{j}+\lambda_{j}-s}-s_{j}^{\beta l_{j}+\lambda_{j}-s}}{r_{j}-s_{j}} .
$$

Введем для любых $k$ и $\rho$ операторы $\theta_{j}^{k, \rho}$ по формуле

$$
\left(\theta_{j}^{k, \rho} f\right)(x)=\frac{r_{j}^{\rho} f\left(r_{j}^{k} x\right)-s_{j}^{\rho} f\left(s_{j}^{k} x\right)}{r_{j}-s_{j}} .
$$


Очевидно,

$$
\theta_{j}^{k, \rho} x^{\beta}=\frac{r_{j}^{k \beta+\rho}-s_{j}^{k \beta+\rho}}{r_{j}-s_{j}} x^{\beta}
$$

Следовательно, функции $v_{j}(\beta)=u_{j}\left(\beta l_{j}+\lambda_{j}-s\right)$ отвечает оператор $\delta_{v_{j}}=\theta_{j}^{l_{j}, \lambda_{j}-s}$. Таким образом, приведенное гипергеометрическое уравнение имеет вид:

$$
\prod_{j: l_{j}>0} \prod_{s=0}^{l_{j}-1} \theta_{j}^{l_{j}, \lambda_{j}-s} F=x \prod_{j: l_{j}<0} \prod_{s=0}^{\left|l_{j}\right|-1} \theta_{j}^{l_{j}, \lambda_{j}-s} F .
$$

Пример. $N=4, l=(-1,-1,1,1)$. В этом случае

$$
v_{i}^{+}(\beta)=u_{3}\left(\beta+\lambda_{3}\right) u_{4}\left(\beta+\lambda_{4}\right), \quad v_{i}^{-}(\beta)=u_{1}\left(-\beta+\lambda_{1}\right) u_{2}\left(-\beta+\lambda_{2}\right) .
$$

Если $u_{i}(\alpha)=\alpha_{i}, i=1,2,3,4$, то приведенное гипергеометрическое уравнение имеет вид:

$$
\left(x \frac{d}{d x}+\lambda_{3}\right)\left(x \frac{d}{d x}+\lambda_{4}\right) F=x\left(-x \frac{d}{d x}+\lambda_{1}\right)\left(-x \frac{d}{d x}+\lambda_{2}\right) F .
$$

Если $\lambda_{3}=0$ или $\lambda_{4}=0$, то оно совпадает с гипергеометрическим уравнением Гаусса. По этой причине естественно рассматривать приведенное гипергеометрическое уравнение $\delta_{w_{+}} F=\delta_{w_{-}} F$ для произвольного набора $u=\left(u_{1}, u_{2}, u_{3}, u_{4}\right)$ функщий $u_{j}$ как $u$-аналог гипергеометрического уравнения Гаусса.

Отметим, что в случае $u_{j}(\alpha)=\frac{r_{j}^{\alpha_{j}}-s_{j}^{\alpha_{j}}}{r_{j}-s_{j}}, j=1,2,3,4$, это уравнение в принятых вьше обозначениях имеет следуюший вид:

$$
\theta_{3}^{1, \lambda_{3}} \theta_{4}^{1, \lambda_{4}} F=x \theta_{1}^{-1, \lambda_{1}} \theta_{2}^{-1, \lambda_{2}} F
$$

6.4. О связи приведенных $u$-гипергеометрических функций с $u$-экспонентами. Описание $u$-гипергеометрических систем и приведенных $u$-гипергеометрических систем упрошается, если их рассматривать как уравнения в пространстве формальных рядов. Именно, пусть $u=\left(u_{1}, \ldots, u_{N}\right)$ - произвольная последовательность не обязательно целых аналитических функций на $\mathbb{C}^{N}$, удовлетворяюших условию (6.1), и $\Sigma \subset \mathbb{C}^{N}-$ множество точек, в которых хотя бы одна из функций $u_{j}$ и $u_{j}^{-1}, j=1, \ldots, N$, имеет особенность. Обозначим через $\mathscr{H}$ пространство, элементы которого - формальные ряды вида

$$
\sum_{\nu \in \mathbb{Z}^{N}} c(\nu) x^{\nu+\lambda}, \text { где } \lambda \notin \Sigma+\mathbb{Z}^{N}
$$

и их конечные линейные комбинации.

На $\mathscr{H}$ естественно определены операторы $\partial_{j}=\partial_{u_{j}, j}$,

$$
\partial_{j} \sum c(\nu) x^{\nu+\lambda}=\partial_{j} \sum c(\nu) u_{j}(\nu+\lambda) a^{\nu+\lambda-e_{j}}, \quad j=1, \ldots, N
$$


причем эти операторы обратимы на $\mathscr{H}$. Таким образом, для каждого $l \in \mathbb{Z}^{N}$ на $\mathscr{H}$ определен оператор

$$
\partial^{l}=\partial_{1}^{l_{1}} \cdots \partial_{N}^{l_{N}}
$$

В результате система уравнений (6.2) может быть представлена в виде:

$$
\partial^{l^{i}} f=f, \quad i=1, \ldots, n,
$$

где $l^{1}, \ldots, l^{n}$ - произвольньй фиксированньй базис решетки $\Lambda$.

Из определения операторов $\partial^{l}$ следует, что на мономы $a^{\alpha}=a_{1}^{\alpha_{1}} \cdots a_{N}^{\alpha_{N}}$ они действуют по формуле

$$
\partial^{l} a^{\alpha}=[u(\alpha)]_{l} a^{\alpha-l}, \quad l \in \mathbb{Z}^{N} .
$$

Поставим в соответствие исходной последовательности $u=\left(u_{1}, \ldots, u_{N}\right)$ функций на $\mathbb{C}^{N}$ последовательность $w=\left(w_{1}, \ldots, w_{n}\right)$ функций на $\mathbb{C}^{n}$, заданных равенствами

$$
w_{i}(\beta)=\left[u\left(\beta_{1} l^{1}, \ldots, \beta_{n} l^{n}+\lambda\right)\right]_{l^{i}}, \quad i=1, \ldots, n,
$$

и положим $\widetilde{\partial}_{i}=\partial_{w_{i}, i}$, т.е. $\widetilde{\partial}_{i}-$ линейный оператор в пространстве функций на $\mathbb{C}^{n}$, действуюший на мономы $x^{\beta}=x_{1}^{\beta_{1}} \cdots x_{n}^{\beta_{n}}$ по формуле

$$
\widetilde{\partial}_{i} x^{\beta}=w_{i}(\beta) x^{\beta-e_{i}}, \quad i=1, \ldots, n .
$$

ПРЕДЛОЖЕНИЕ 6.4. Приведенная и-гипергеометрическая система, ассоциированная с подрешеткой $\Lambda \subset \mathbb{Z}^{N}$ и вектором $\lambda \in \mathbb{C}^{N}$, имеет вид

$$
\widetilde{\partial}_{i} F=F, \quad i=1, \ldots, n .
$$

Таким образом, ее формальные решения в пространстве рядов суть w-экспонен$m b l$.

ДоКАЗАТЕЛЬСТво. Подставив $f(a)=a^{\lambda} F(x)$ в (6.11), получаем на основании (6.12) следуюшую систему уравнений для $F: D_{i} F=F, i=1, \ldots, n$, где $D_{i}$ действует на мономы $a^{\alpha}=a_{1}^{\alpha_{1}} \cdots a_{N}^{\alpha_{N}}$ по формуле

$$
D_{i} a^{\alpha}=[u(\alpha+\lambda)]_{l^{i}} a^{\alpha-l^{i}} .
$$

Остается убедиться, что $D_{i} x^{\beta}=w_{i}(\beta) x^{\beta-e_{i}}$. В самом деле, так как $x_{i}=a^{l^{i}}, i=$ $1, \ldots, n$, то $x^{\beta}=a^{\beta_{1} l^{1}+\cdots+\beta_{n} l^{n}}$, а потому

$D_{i} x^{\beta}=D_{i} a^{\beta_{1} l^{1}+\cdots+\beta_{n} l^{n}}=\left[u\left(\beta_{1} l^{1}+\cdots+\beta_{n} l^{n}+\lambda\right)\right]_{l^{i}} a^{\beta_{1} l^{1}+\cdots+\beta_{n} l^{n}-l^{i}}=w_{i}(\beta) x^{\beta-e_{i}}$.

6.5. Решения $u$-гипергеометрических систем в форме Г-рядов. Рассмотрим $u$-гипергеометрическую систему $(6.2),(6.3)$, ассоциированную с произвольной подрешеткой $\Lambda \subset \mathbb{Z}^{N}$ и вектором $\beta \in \mathbb{C}^{N} / L$. 
ПРЕДЛОЖЕНИЕ 6.5. Если $\gamma \in \mathbb{C}^{N}$ принадлежит прообразу вектора $\beta \in \mathbb{C}^{N} / L$ u $E_{u}(l+\gamma) \neq 0$ при $l \in \Lambda$, то формальньй Г-ряд

$$
f_{\Lambda}(\gamma, a)=\sum_{l \in \Lambda} \frac{a^{l+\gamma}}{E_{u}(l+\gamma)}
$$

формально удовлетворяет системе (6.2), (6.3).

ДоКАЗАТЕЛЬСТВо. Имеем для Любого $\omega \in \Lambda^{+}$:

$$
\sum_{i=1}^{N} \omega_{i} a_{i} \frac{\partial f_{\Lambda}}{\partial a_{i}}=\sum_{l \in \Lambda}\langle\omega, l+\gamma\rangle \frac{a^{l+\gamma}}{E_{u}(l+\gamma)}=\langle\omega, \beta\rangle f
$$

Убедимся, далее, что для любого $l=\left(l_{1}, \ldots, l_{N}\right)$ с неотрицательными координатами и любого $\gamma \in \mathbb{C}^{N}$, для которого $E_{u}(\gamma) \neq 0$, справедливо равенство

$$
\partial^{l}\left(\frac{a^{\gamma}}{E_{u}(\gamma)}\right)=\frac{a^{\gamma-l}}{E_{u}(\gamma-l)}, \quad \text { где } \quad \partial^{l}=\partial_{1}^{l_{1}} \cdots \partial_{N}^{l_{N}} .
$$

В самом деле, имеем:

$$
\partial^{l} a^{\gamma}=[u(\gamma)]_{l} a^{\gamma-l}, \quad E_{u}(\gamma)=[u(\gamma)]_{l} E_{u}(\gamma-l)
$$

Так как $l$ - вектор с неотрицательными координатами, то $[u(\gamma)]_{l}<\infty$. Поэтому, поскольку $E_{u}(\gamma) \neq 0$, возможны только следующие варианты:

$$
\text { 1) } \left.[u(\gamma)]_{l}=0, \quad E_{u}(\gamma-l)=\infty ; \quad 2\right)[u(\gamma)]_{l} \neq 0, \quad E_{u}(\gamma-l) \neq 0
$$

В случае 1) $a^{\gamma} / E_{u}(\gamma)=0$, апотому $\partial^{l}\left(a^{\gamma} / E_{u}(\gamma)\right)=0=a^{\gamma-l} / E_{u}(\gamma-l)$. В случае 2) $\partial^{l}\left(a^{\gamma} / E_{u}(\gamma)\right)=[u(\gamma)]_{l} a^{\gamma-l} / E_{u}(\gamma)=a^{\gamma-l} / E_{u}(\gamma-l)$.

Из (6.13) следует, что для любого $l \in \mathbb{Z}^{N}$

$$
\prod_{j=1}^{N} \partial_{j}{ }^{l_{j}^{+}} f_{\Lambda}(\gamma, a)=f_{\Lambda}\left(\gamma-l^{+}, a\right), \quad \prod_{j=1}^{N} \partial_{j}{ }^{-} f_{\Lambda}(\gamma, a)=f_{\Lambda}\left(\gamma-l^{-}, a\right) .
$$

Если $l \in \Lambda$, то $\left(\gamma-l^{-}\right)-\left(\gamma-l^{+}\right) \in \Lambda$, а потому $f_{\Lambda}\left(\gamma-l^{+}, a\right)=f_{\Lambda}\left(\gamma-l^{-}, a\right)$. Значит, вьполняются условия (6.3). Предложение доказано.

Пусть теперь $l^{1}, \ldots, l^{n}-$ произвольный фиксированньй базис в $\Lambda$, т.е.

$$
\Lambda=\left\{k_{1} l^{1}+\cdots+k_{n} l^{n} \mid k \in \mathbb{Z}^{N}\right\},
$$

и $\lambda \in \mathbb{C}^{N}$ - фиксированньй вектор в прообразе $\beta \in \mathbb{C}^{N} / L$.

Пусть $x=\left(x_{1}, \ldots, x_{n}\right)$, где $x_{i}=a^{l^{i}}$, и $v^{\gamma}=\left(v_{1}, \ldots, v_{n}\right)$ - последовательность функций на $\mathbb{C}^{n}$, заданных равенствами

$$
v_{i}(\beta)=\left[u\left(\beta_{1} l^{1}+\cdots+\beta_{n} l^{n}+\gamma\right)\right]_{l^{i}} .
$$


ПРЕДЛОЖЕНИЕ 6.6. Для любого вектора $\gamma \in \mathbb{C}^{N}$ из прообраза $\beta \in \mathbb{C}^{N} / L$ ряд $f_{\Lambda}(\gamma, a)$ представим в виде

$$
f_{\Lambda}(\gamma, a)=a^{\lambda} x^{\gamma^{\prime}} \exp _{v^{\gamma}} x
$$

əде $\gamma^{\prime}=\left(\gamma_{1}^{\prime}, \ldots, \gamma_{n}^{\prime}\right)$ определяется из разложения $\gamma-\lambda=\sum_{i=1}^{n} \gamma_{i}^{\prime} l^{i}$.

ДокАЗАТЕльство. Из предложения 5.3 следует, что $f_{\Lambda}(\gamma, a)=a^{\gamma} \exp _{v} x$. Остается заметить, что $a^{\gamma}=a^{\lambda} a^{\sum \gamma_{i}^{\prime} l^{i}}$.

СлЕДСТВИЕ. Формальные ряды

$$
F_{\Lambda}(\gamma, a)=x^{\gamma^{\prime}} \exp _{v^{\gamma}} x
$$

формально удовлетворяют приведенной и-гипергеометрической системе (6.9), ассоииированной с $\Lambda \subset \mathbb{Z}^{N} u \beta \in \mathbb{C}^{N} / L$.

Из предложения 6.6 следует, что ряды $f_{\Lambda}(\gamma, a)$ и $F_{\Lambda}(\gamma, a)$, где $\gamma \in \mathbb{C}^{N}$ принадлежит прообразу вектора $\beta \in \mathbb{C}^{N} / L$, задают в области своей сходимости соответственно $u$-гипергеометрическую функцию и приведенную $u$-гипергеометрическую функцию. Условия сходимости некоторых Г-рядов были обсуждены в $\S 5$.

6.6. Решения $u$-гипергеометрических систем в форме интегралов. Рассмотрим $u$-гипергеометрическую систему $(6.2),(6.3)$ для $u=\left(u_{1}, \ldots, u_{N}\right)$, где каждая функция $u_{j}, j=1, \ldots, N$, зависит только от $\alpha_{j}$. Фиксируем произвольньй базис

$$
\omega_{j}=\left(\omega_{j}^{1}, \ldots, \omega_{j}^{N}\right), \quad j=1, \ldots, r
$$

$r=\operatorname{rank} \Lambda^{\perp}$, в подпространстве $L_{\mathbb{R}}^{\perp} \subset\left(\mathbb{R}^{N}\right)^{\prime}$, ортогональном $\Lambda$, и обозначим:

$$
\beta_{j}=\left\langle\omega_{j}, \beta\right\rangle, \quad j=1, \ldots, r
$$

Тогда система уравнений (6.3) эквивалентна следуюшей:

$$
\sum_{i=1}^{N} \omega_{j}^{i} a_{i} \frac{\partial f}{\partial a_{i}}=\beta_{j} f, \quad j=1, \ldots, r .
$$

Рассмотрим следуюшие интегралы:

$$
F(a)=\int_{C} \prod_{i=1}^{N} f_{u_{i}}\left(a_{i} t^{\omega^{i}}\right) \prod_{j=1}^{r} t_{j}^{-\beta_{j}-1} d t_{j}
$$

где $f_{u_{j}}(s)$ - фиксированная $u_{j}$-экспонента, т.е. решение уравнения $\partial_{u_{j}} f=f, j=$ $1, \ldots, N, t^{\omega^{i}}=t_{1}^{\omega_{1}^{i}} \cdots t_{r}^{\omega_{r}^{i}}$. Интеграл берется по области

$$
C=\left\{t \in \mathbb{C}^{r} \mid \arg t_{j}=\text { const }, j=1, \ldots, r\right\} .
$$


ПРЕДЛОЖЕНИЕ 6.7. Интеграл (6.15) формально удовлетворяет и-гипергеометрической системе (6.2), (6.3).

ДоКАЗАТЕЛЬСТво. Воспользуемся тем, что операторы $\partial_{u_{j}}$ обладают свойством: если $\varphi(s)=f(c s)$, то $\left(\partial_{u_{j}} \varphi\right)(s)=c\left(\partial_{u_{j}} f\right)(c s)$. Отсюда для любого $l \in \mathbb{Z}^{N}$ следует:

$$
\begin{aligned}
& \prod_{j=1}^{N} \partial_{j}{ }^{l_{j}^{+}} F(a)=\int_{C} t^{\omega^{+}} \prod_{i=1}^{N} f_{u_{i}}\left(a_{i} t^{\omega^{i}}\right) \prod_{j=1}^{r} t_{j}^{-\beta_{j}-1} d t_{j}, \\
& \prod_{j=1}^{N} \partial_{j}{ }^{l_{j}^{-}} F(a)=\int_{C} t^{\omega^{-}} \prod_{i=1}^{N} f_{u_{i}}\left(a_{i} t^{\omega^{i}}\right) \prod_{j=1}^{r} t_{j}^{-\beta_{j}-1} d t_{j},
\end{aligned}
$$

где $\omega^{+}=\sum_{i=1}^{N} l_{i}^{+} \omega^{i}, \omega^{-}=\sum_{i=1}^{N} l_{i}^{-} \omega^{i}$. Поскольку $\omega_{j}^{+}-\omega_{j}^{-}=\left\langle l^{+}-l^{-}, \omega_{j}\right\rangle=\left\langle l, \omega_{j}\right\rangle$, $j=1, \ldots, r$, то $\omega^{+}=\omega^{-}$для любого $l \in \Lambda$. Следовательно, при $l \in \Lambda$ правые части равенств (6.17) совпадают, и, значит, $F(a)$ удовлетворяет уравнению (6.2).

Убедимся теперь, что

$$
F\left(\lambda^{\omega_{j}} a\right)=\lambda^{\beta_{j}} F(a), \quad j=1, \ldots, r,
$$

для любого $\lambda>0$, где $\lambda^{\omega_{j}} a=\left(\lambda^{\omega_{j}^{1}} a_{1}, \ldots, \lambda^{\omega_{j}^{N}} a_{N}\right)$. В самом деле,

$$
F\left(\lambda^{\omega_{j}} a\right)=\int_{C} \prod_{i=1}^{N} f_{u_{i}}\left(a_{i} \lambda^{\omega_{j}^{i}} t^{\omega^{i}}\right) \prod_{s=1}^{r} t_{s}^{-\beta_{s}-1} d t_{s} .
$$

Поскольку $\lambda^{\omega_{j}^{i}} t^{\omega^{i}}=t_{1}^{\omega_{1}^{i}} \cdots\left(\lambda t_{j}\right)^{\omega_{j}^{i}} \cdots t_{r}^{\omega_{r}^{i}}$, то заменой переменной $\lambda t_{j} \mapsto t_{j}$ интеграл справа приводится к виду $\lambda^{\beta_{j}} F(a)$. Из равенств (6.18) следуют уравнения (6.14).

СЛЕДСТВИЕ. Если интеграл (6.15) сходится в некоторой области пространства $\mathbb{C}^{N}$, то он задает в этой области и-гипергеометрическую функцию, ассоциированную с подрешеткой $\Lambda \subset \mathbb{Z}^{N}$ и вектором $\beta \in \mathbb{C}^{N} / L$.

ЗАмЕчАНИЕ. Предложение 6.7 остается справедливым для любого "цикла" $C \subset \mathbb{C}^{N}$ вешественной размерности $r$.

ПРИмеР. Рассмотрим случай, когда функции $u_{j}(\alpha)$ имеют вид

$$
u_{i}(\alpha)=\frac{q_{i}^{\alpha}-1}{q_{i}-1}, \quad 0<q_{i}<1, \quad i=1, \ldots, N .
$$

В этом случае в качестве $u_{i}$-экспоненты можно взять функцию $f_{u_{i}}(t)=\exp _{q_{i}}(t)$, введенную в п. 2.3, и интеграл (6.15) принимает вид

$$
F(a)=\int_{C} \prod_{i=1}^{N} \exp _{q_{i}}\left(a_{i} t^{\omega^{i}}\right) \prod_{i=1}^{r} t_{i}^{-\beta_{i}-1} d t_{i} .
$$

Убедимся, что этот интеграл сходится в некоторой области $U \subset \mathbb{C}^{N}$. Сначала представим (6.19) в более простой форме. Фиксируем в $\mathbb{R}^{N}$ произвольное координатное 
подпространство размерности $r$, имеющее нулевое пересечение с $L \subset \mathbb{C}^{N}$. Пусть для определенности это будет подпространство, натянутое на векторы $e_{1}, \ldots, e_{r}$. Тогда в подпространстве $L_{\mathbb{R}}^{\perp} \subset\left(\mathbb{R}^{N}\right)^{\prime}$, ортогональном к $L$, существует и однозначно определен базис

$$
\omega_{i}=\left(\omega_{i}^{1}, \ldots, \omega_{i}^{N}\right), \quad i=1, \ldots, r
$$

такой, что $\omega_{i}^{j}=\delta_{i}^{j}, i, j=1, \ldots, r$. По отношению к этому базису интеграл (6.19) имеет следуюший вид:

$$
F(a)=\int_{C} \prod_{i=1}^{r} \exp _{q_{i}}\left(a_{i} t_{i}\right) \prod_{i=r+1}^{N} \exp _{q_{i}}\left(x_{i} t^{\omega^{i}}\right) \prod_{i=1}^{r} t_{i}^{-\beta_{i}-1} d t_{i}
$$

ЗАмЕчАнИЕ. Интеграл (6.20) получается из (6.19) заменой переменных: $t^{\omega^{i}} \mapsto t_{i}$, $i=1, \ldots, r$.

ПРЕДЛОЖЕНИЕ 6.8. Пусть $C=\left\{t \in \mathbb{C}^{r} \mid \arg t_{j}=\varphi_{j}, j=1, \ldots, r\right\}$, әде $\varphi_{j}-$ произвольные фиксированнье числа, и пусть $\operatorname{Re} \beta_{j}<0, j=1, \ldots, r$. Тогда интеграл (6.20) сходится для любого а $\in \mathbb{C}^{N}$ с отличными от нуля координатами такого, ито на $C \arg \left(a_{j} t_{j}\right) \neq 0, j=1, \ldots, r, u \arg \left(a_{i} t^{\omega^{i}}\right) \neq 0, i=r+1, \ldots, N$.

ДокАЗАТЕльство. Заметим, что функции $\exp _{q_{i}} s$ регулярны в точке $s=0$, и на каждом луче, отличном от вешественной положительной полуоси, они ограничены. Отсюда следует, что доказательство сходимости интеграла (6.20) сводится к доказательству сходимости интегралов

$\int_{\arg t_{j}=\varphi_{j}} \exp _{q_{j}}\left(a_{j} t_{j}\right) t_{j}^{-\beta_{j}-1} d t_{j}=a_{j}{ }^{\beta_{j}} \int_{\arg t_{j}=\varphi_{j}^{\prime}}\left(\exp _{q_{j}} t_{j}\right) t_{j}^{-\beta_{j}-1} d t_{j}, \quad j=1, \ldots, r$, где $\varphi_{j}^{\prime} \neq 0$.

Сходимость этих интегралов непосредственно следует из оценки, полученной в п. 2.3: $\left|\exp _{q_{j}} t_{j}\right|=\exp \left\{\frac{1}{2} \frac{\ln ^{2}\left|t_{j}\right|}{\ln q_{j}}+O\left(\ln \left|t_{j}\right|\right)\right\}$ при $\left|t_{j}\right| \rightarrow \infty$ на каждом луче, отличном от положительной вешественной полуоси.

ЗАмЕчАниЕ. Для таких функций $u_{i}$ решений $u$-гипергеометрической системы, заданных сходяшимися Г-рядами, вообще говоря, не существует. Например, согласно п. 5.4 не сушествует сходяшихся $Г$-рядов в случае, когда $\Lambda$ - подрешетка в $\mathbb{Z}^{4}$, порожденная вектором $l=(-1,-1,1,1)$. 


\section{СПИСОК ЛИТЕРАТУРЫ}

[1] Гельфанд И. М. Общая теория гипергеометрических функций // Докл. АН СССР. 1986. T. 288. № 1. C. $14-18$

[2] Гельфанд И.М., Гельфанд С.И. Обобшенные гипергеометрические уравнения // Докл. АН СССР. 1986. Т. 288. № 2. С. 279-283.

[3] Гельфанд И. М., Граев М. И., Зелевинский А. В. Голономные системы уравнений и ряды гипергеометрического типа // Докл. АН СССР. 1987. Т. 295. № 1. С. 14-19.

[4] Гельфанд И. М., Зелевинский А.В., Капранов М. М. Гипергеометрические функции и торические многообразия // Функцион. анализ и его прилож. 1989. Т. 23. № 2. C. $12-26$.

[5] Гельфанд И.М., Граев М.И., Ретах В.С. Общие гипергеометрические системы уравнений и ряды гипергеометрического типа // УМН. 1992. Т. 47. № 4. С. 3-82.

[6] Гельфанд И.М., Граев М.И., Ретах В.С. q-гипергеометрическое уравнение Гаусса и описание его решений в виде рядов и интегралов // Докл. АН. 1993. Т. 331. № 2. C. $140-143$.

[7] Гельфанд И.М., Граев М.И., Ретах В.С. $(R, S)$-гипергеометрические функции одной переменной // Докл. АН. 1993. Т. 333. № 5. С. 567-570.

[8] Гельфанд И. М., Граев М.И., Ретах В.С., Спирин С. А. $(r, s)$-экспоненты // Докл. АН. 1994. Т. 336. №6. С. 730-732.

[9] Граев М.И. Уравнения и ряды гипергеометрического типа // Функцион. анализ и его прилож. 1995. Т. 29. №1. С. 72-76.

[10] Бейтмен Г., Эрдейи А. Высшие трансцендентные функции. Т. 1. М.: Наука, 1973.

[11] Agarwal R. P. Pade approximants, continued fractions and Heine $q$-hypergeometric series // J. Math. Phys. Sci. 1992. V. 26. № 3. P. 281-290.

[12] Al-Salam W. A., Verma A. On quadratic transformations of basic series // SIAM J. Math. Anal. 1984. V. 15. P. 414-420.

[13] Andrews G. E. On the $q$-analogue of Kummer's theorem and applications // Duke Math. J. 1973. V. 40. P. 525-528.

[14] Andrews G. E. On $q$-analogues of the Watson and Whipple summations // SIAM J. Math. Anal. 1976. V. 7. P. 332-336.

[15] Andrews G. E. $q$-series: Their Development and Application in Analysis, Number Theory, Combinatorics, Physics, and Computer Algebra (CBMS Regional Conference Series in Math.). V. 66. Providence, RI: Amer. Math. Soc., 1986.

[16] Andrews G. E. Problems and prospects for basic hypergeometric functions // Theory and Applications of Spherical Functions. New York: Academic Press, 1995. P. 191-224.

[17] Andrews G.E., Askey R. Another q-extension of the beta function // Proc. Amer. Math. Soc. 1981. V. 81. P. 97-100.

[18] Aomoto K. Connection formulas in the $q$-analog de Rham cohomology // Progr. Math. 1995. V. 131. P. 1-12.

[19] Aomoto K. On connection coefficients for $q$-difference systems of A-type Jackson integrals // SIAM J. Math. Anal. 1994. V. 25. № 2. P. 256-273.

[20] Aomoto K., Kato Y. A $q$-analogue of de Rham cohomology associated with Jackson integrals // Special Functions, Proc. Hayashibara Forum (Okayama, 1990), ICM-90 Satell. Conf. Proc., 1991. P. 30-62.

[21] Aomoto K., Mimachi Y. A solution of the Yang-Baxter equation as connection coefficients of a holonomic $q$-difference system // Internat. Math. Res. Notices. 1992. № 1. P. 7-15.

[22] Askey R., Suslov S. K. The $q$-harmonic oscillator and an analogue of the Charlier polynomials // J. Phys. A. 1993. V. 26. № 15. P. L693-L698.

[23] Askey R., Wilson J. A. Some basic hypergeometric orthogonal polynomials that generalize Jacobi polynomials // Mem. Amer. Math. Soc. 1985. V. 319. P. 1-55.

[24] Buschman R. G. Contiguous function relations for $q$-hypergeometric functions // Indian J. Math. 1994. V. 36. № 1. P. 79-84.

[25] Carlitz L. Some formulas of F. H. Jackson // Monatsh. Math. 1969. V. 73. P. 193-198. 
[26] Dwork B. Generalized Hypergeometric Functions. Oxford: Clarendon Press, 1990.

[27] Exton H. A theorem of transformations of multiple $q$-hypergeometric functions // J. Indian Acad. Math. 1994. V. 16. № 2. P. 201-204.

[28] Floreanini R., Vinet L. On the quantum group and quantum algebra approach to $q$-special functions // Lett. Math. Phys. 1993. V. 27. № 3. P. 179-190.

[29] Floreanini R., Vinet L. $q$-conformal quantum mechanics and $q$-spherical functions // Phys. Lett. B. 1992. V. 277. № 4. P. 442-446.

[30] Floreanini R., Vinet L. Quantum algebra approach to $q$-Gegenbauer polynomials // J. Comput. Appl. Math. 1995. V. 57. № 1-2. P. 123-133.

[31] Floreanini R., Vinet L. Using quantum algebras in $q$-special functions theory // Phys. Lett. A. 1992. V. 170. № 1. P. 21-28.

[32] Floreanini R., Morozov A., Vinet L. $q$-hypergeometric functions, quantum algebras and free fields // Teor. Mat. Fizika. 1994. V. 98. № 3. P. 379-387.

[33] Frenkel I. B., Reshetikhin N.Y. Quantum affine algebras and holonomic difference equations // Comm. Math. Phys. 1992. V. 146. № 1. P. 1-60.

[34] Gasper G. Rogers' linearization formula for the continuous $q$-ultraspherical polynomials and quadratic transformations formulas // SIAM J. Math. Anal. 1985. V. 16. P. 1061-1071.

[35] Gasper G., Rahman M. Basic Hypergeometric Series. Cambridge: Cambridge Univ. Press, 1990.

[36] Gasper G., Rahman M. Positivity of the Poisson kernel for the continuous $q$-Jacoby polynomials and some quadratic transformation formulas for basic hypergeometric series // SIAM J. Math. Anal. 1986. V. 17. P. 970-999.

[37] Gasper G., Rahman M. Product formulas of Watson, Bailey and Bateman types and positivity of the Poisson kernel for $q$-Racah polynomials // SIAM J. Math. Anal. 1984. V. 15. P. 768-789.

[38] Gelfand I. M., Kapranov N. M., Zelevinsky A. V. Discriminants, Resultants and Multidimensional Determinants. Boston: Birkhäuser, 1994.

[39] Groza V.A. Quantum algebras, Kravchuk $q$-polynomials, and Kravchuk-Meixner $q$-functions // Укр. матем. журн. 1992. V. 44. № 7. Р. 983-986.

[40] Gupta A. On Certain bilateral bibasic $q$-hypergeometric series transformations and summations // Proc. Nat. Acad. Sci. India. Sect. A 1992. V. 62. № 1. P. 87-96.

[41] Horikawa E. Contiguity relations for $q$-hypergeometric functions and related quantum groups // Proc. Japan Acad. Ser. A. 1992. V. 68. № 7. P. 157-160.

[42] Jain V.K. Certain transformations of basic hypergeometric series and their applications // Pacific J. Math. 1982. V. 101. P. 333-349.

[43] Jain V.K. Some transformations of basic hypergeometric functions // SIAM J. Math. Anal. 1981. V. 12. P. 957-961.

[44] Jain V.K., Verma A. Some transformations of basic hypergeometric functions // SIAM J. Math. Anal. 1981. V. 12. P. 943-956.

[45] Jing N. H. q-hypergeometric series and Macdonald functions // J. Algebraic Combin. 1994. V. 3. № 3. P. 291-305.

[46] Kalnins E. G., Miller W., Mukherjee S. Models of $q$-algebra representations: matrix elements of $U_{q}(s u(2)) / /$ Contemp. Math. 1994. V. 160. P. 191-208.

[47] Koelnik H.T. The quantum group of plane motions and the Hahn-Exton $q$-Bessel function // Duke Math. J. 1994. V. 76. № 2. P. 483-508.

[48] Koornwinder T. H. Orthogonal polynomials in connection with quantum groups // Orthogonal Polynomials: Theory and Practice (Proc. NATO ASI, Colombus, USA, 1989). NATO ASI Ser. V. 294, 1990. P. 257-292.

[49] Koornwinder T.H. Representations of the twisted $S U(2)$ quantum group and some $q$-hypergeometric orthogonal polynomials // Indagationes Math. 1989. V. 51. № 1. P. 97-117.

[50] Masuda T., Mimachi K., Nakagami Y., Noumi M., Ueno K. Representations of quatum groups and a $q$-analogue of orthogonal polynomials // C. R. Acad. Sci. Paris. Ser. I. 1988. V. 307. P. 559-564. 
[51] Mathai A. M., Saxena R. K. The $H$-functions with Applications in Statistics and Other Disciplines. New York: Halsted Press, Wiley, 1978.

[52] McIntosh R. J. Some asymptotic formulae for $q$-hypergeometric series // J. London Math. Soc. 1995. V. 51. № 1. P. 120-136.

[53] Mimachi K., Noumi M. Quantum 2-spheres and big $q$-Jacobi polynomials // Comm. Math. Phys. 1990. V. 128. P. 521-531.

[54] Morozov A., Vinet L. $q$-hypergeometric functions in the formalism of free fields // Modern Phys. Lett. A. 1993. V. 8. № 30. P. 2891-2902.

[55] Noumi M. Quantum Grassmanians and $q$-hypergeometric series // CWI-Quarterly. 1992. V. 5. № 4. P. 293-307.

[56] Rahman M., Verma A. Quadratic transformations formulas for basic hypergeometric series // Trans. Amer. Math. Soc. 1993. V. 335. № 1. P. 277-302.

[57] Sato M., Shintani T., Muro M. Theory of prehomogeneous vector spaces. Singular orbits of a prehomogeneous vector space and hypergeometric functions // Nagoya Math. J. 1990. V. 120. P. 1-34.

[58] Slater L. Generalized Hypergeometric Functions. Cambridge: Cambridge Univ. Press, 1966.

[59] Srivastava H. M., Karlsson P.-W. Multiple Gaussian Hypergeometric Series. Chicester: Wiley, 1985.

[60] Srivastava H. M., Singh S. N., Shukla H.S. Transformations of certain generalized $q$-hypergeometric functions of two variables // J. Math. Anal. Appl. 1995. V. 196. № 2 . P. 554-565.

[61] Varchenko A. N. Quantized Knizhnik-Zamolodchikov equations, quantized Yang-Baxter equation and difference equations for $q$-hypergeometric functions // Comm. Math. Phys. 1994. V. 162. № 3. P. 499-528.

[62] Yang K. W. Matrix $q$-hypergeometric series // Discrete Math. 1995. V. 146. № 1-3. P. 271-284. 